Projet OCDE/G20 sur l'érosion de la base d'imposition et le transfert de bénéfices

\title{
Instructions relatives
} aux aspects intéressant les prix de transfert des actifs incorporels

\section{ACTION 8 : Livrable 2014}

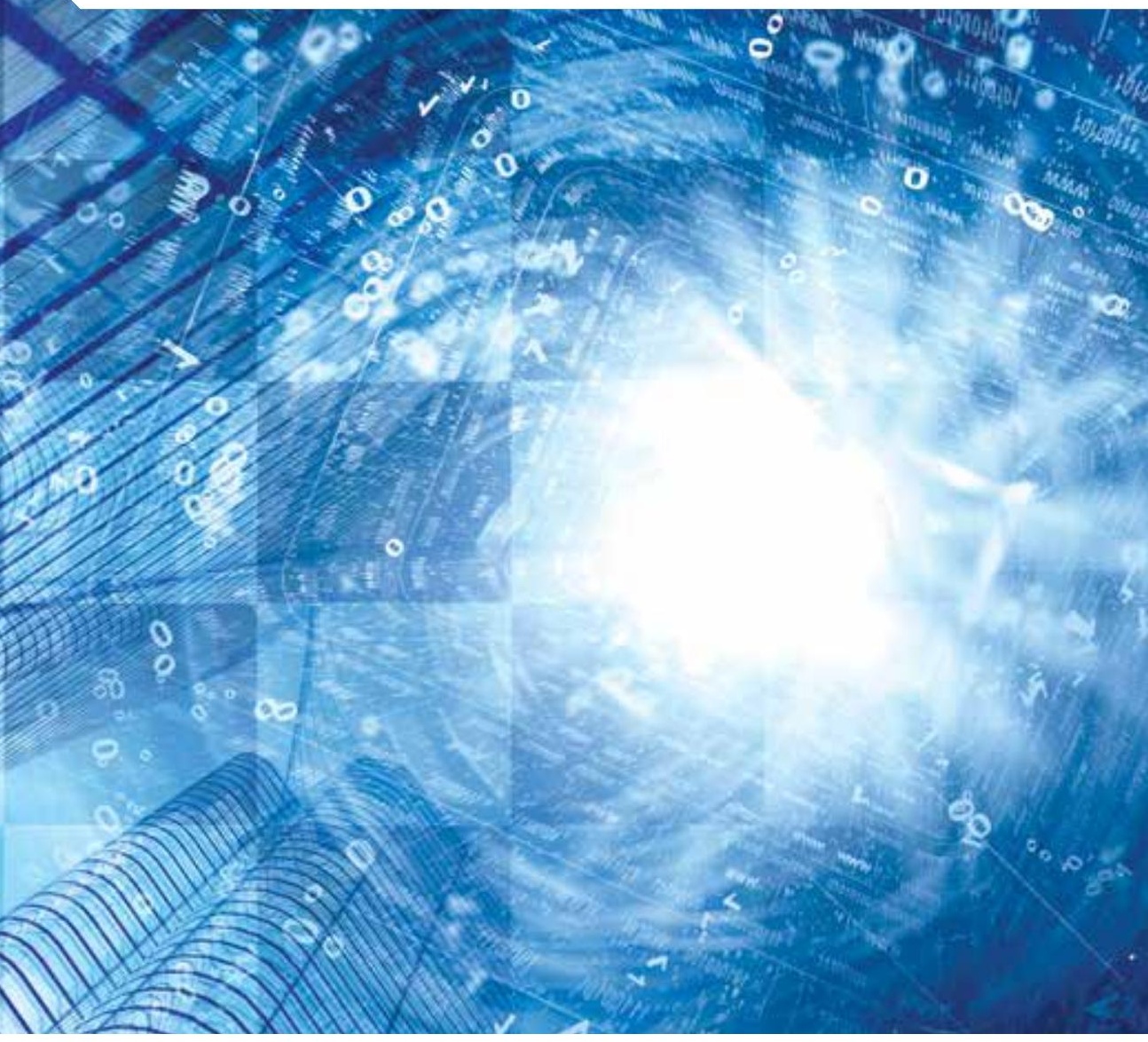

(2) $) O C D E$ 

Projet OCDE/G20 sur l'érosion de la base d'imposition et le transfert de bénéfices

\section{Instructions relatives aux aspects intéressant les prix de transfert des actifs incorporels}

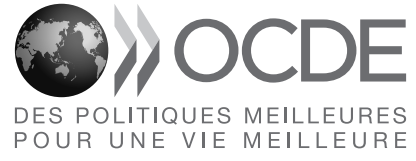


Ce document et toute carte qu'il peut comprendre sont sans préjudice du statut de tout territoire, de la souveraineté s'exerçant sur ce dernier, du tracé des frontières et limites internationales, et du nom de tout territoire, ville ou région.

Merci de citer cet ouvrage comme suit :

OCDE (2014), Instructions relatives aux aspects intéressant les prix de transfert des actifs incorporels, Projet OCDE/G20 sur l'érosion de la base d'imposition et le transfert de bénéfices, Éditions OCDE.

http://dx.doi.org/10.1787/9789264225350-fr

ISBN 978-92-64-22533-6 (imprimé)

ISBN 978-92-64-22535-0 (PDF)

Collection : Projet OCDE/G20 sur l'érosion de la base d'imposition et le transfert de bénéfices

ISSN 2313-2620 (imprimé)

ISSN 2313-2639 (en ligne)

Crédits photo : Couverture $\odot$ archerix / Fotolia.

Les corrigenda des publications de l'OCDE sont disponibles sur:

www.oecd.org/about/publishing/corrigenda.htm.

(C) OCDE 2014

La copie, le téléchargement ou l'impression du contenu OCDE pour une utilisation personnelle sont autorisés. Il est possible d'inclure des extraits de publications, de bases de données et de produits multimédia de l'OCDE dans des documents, présentations, blogs, sites Internet et matériel pédagogique, sous réserve de faire mention de la source et du copyright. Toute demande en vue d'un usage public ou commercial ou concernant les droits de traduction devra être adressée à rights@oecd.org. Toute demande d'autorisation de photocopier une partie de ce contenu à des fins publiques ou commerciales devra être soumise au Copyright Clearance Center (CCC), info@copyright.com, ou au Centre français d'exploitation du droit de copie (CFC), contact@cfcopies.com. 


\section{Avant-propos}

Enrayer l'érosion de la base d'imposition et le transfert de bénéfices (BEPS) est une priorité absolue pour les pouvoirs publics partout dans le monde. En 2013, les pays de l'OCDE et du G20, œuvrant sur un pied d'égalité, ont adopté un Plan d'action en 15 points visant à lutter contre l'érosion de la base d'imposition et le transfert de bénéfices. Le Plan d'action vise à faire coïncider le lieu d'imposition des bénéfices avec le lieu d'exercice des activités économiques à l'origine de ces bénéfices et de la création de valeur. Lutter contre l'érosion de la base d'imposition et le transfert de bénéfices constitue un impératif pour tous les pays et il convient d'agir rapidement, notamment pour empêcher que ne se défasse le cadre fiscal international existant fondé sur le consensus, ce qui aggraverait l'incertitude pour les entreprises, à l'heure où les investissements internationaux sont plus nécessaires que jamais. Par conséquent, le Plan d'action prévoit 15 mesures à mettre en œuvre d'ici à la fin de 2015 et, pour certaines d'entre elles, dès 2014.

Le Comité des affaires fiscales (CAF) de l'OCDE, qui réunit 44 pays sur un pied d'égalité (tous les membres de l'OCDE, les pays du G20 et les pays en voie d'adhésion à l'OCDE), a adopté un premier ensemble de sept rapports et recommandations décrits dans le Plan d'action et dus en 2014. Le présent rapport fait partie de ces réalisations et relève de l'Action 8.

Les pays en développement et d'autres économies non membres de l'OCDE ou du G20 ont été largement consultés au cours de nombreuses réunions régionales et d'autres forums mondiaux, et leurs contributions ont été prises en compte dans les travaux. Les représentants d'entreprises, syndicats, organisations de la société civile et universitaires ont également pris une part active au processus, saisissant l'occasion qui leur était donnée de commenter les documents de travail. Plus de 3500 pages de commentaires ont ainsi été reçues et débattues au cours de cinq réunions publiques de consultation et de trois sessions interactives diffusées sur le Web, qui ont attiré près de 10000 visiteurs.

Le premier ensemble de rapports et de recommandations, élaborés en 2014, constitue une réponse à sept des quinze points du Plan d'action publié 
en juillet 2013. L'objectif du Plan d'action étant d'offrir des solutions complètes et cohérentes pour lutter contre les pratiques d'érosion de la base d'imposition et le transfert de bénéfices, les mesures proposées, bien qu'approuvées sur le principe, ne sont pas encore finalisées. Elles sont susceptibles d'évoluer au gré de certaines décisions prises au regard des résultats attendus pour 2015, auxquels elles sont étroitement liées. Elles reflètent toutefois le consensus, en date de juillet 2014, qui entoure un certain nombre de solutions adoptées pour mettre un terme à l'érosion de la base d'imposition et le transfert de bénéfices.

L'adoption de ce premier ensemble de rapports et de recommandations et la mise en œuvre des mesures pertinentes par les autorités nationales permettront : de neutraliser les effets des montages hybrides ; de s'attaquer au chalandage fiscal et à d'autres formes d'utilisation abusive des conventions ; de réduire significativement la manipulation des règles de détermination des prix de transfert dans le domaine sensible des biens incorporels ; et de mettre en place des déclarations pays par pays qui donneront aux pouvoirs publics des renseignements sur la répartition mondiale des bénéfices, des activités économiques et des impôts acquittés par les entreprises multinationales. Par ailleurs, les pays de l'OCDE et du G20 ont adopté un rapport qui conclut à la faisabilité de la mise en œuvre des mesures arrêtées dans le cadre du Projet BEPS par le biais d'un instrument multilatéral. Ils ont également progressé dans la lutte contre les pratiques fiscales dommageables, notamment dans le domaine des régimes de la propriété intellectuelle et des rescrits fiscaux. Enfin, ils sont parvenus à une compréhension commune des défis posés par l'économie numérique, ce qui leur permettra d'approfondir leurs travaux dans ce domaine où les phénomènes d'érosion de la base d'imposition et le transfert de bénéfices sont exacerbés.

Par nature, les pratiques d'érosion de la base d'imposition et le transfert de bénéfices appellent des réponses coordonnées. C'est pourquoi les pays consacrent du temps et des ressources à forger des solutions communes à des problèmes communs. Dans le même temps, ils conservent leur souveraineté fiscale et sont libres d'appliquer les mesures selon des modalités différentes, dès lors qu'elles ne vont pas à l'encontre de leurs engagements juridiques internationaux. 


\section{Table des matières}

Abréviations et acronymes............................................................................................7

Synthèse .........................................................................................................9

Modifications apportées aux chapitres I et II des Principes applicables en matière de prix de transfert .........................................................15

D.6. Économies de localisation et caractéristiques du marché local.............15

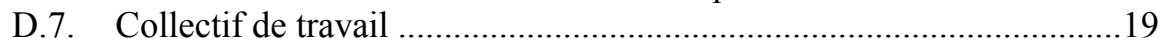

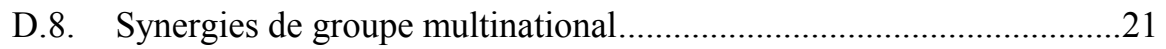

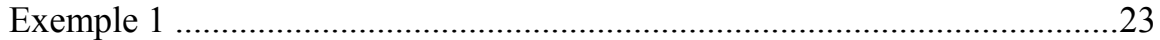

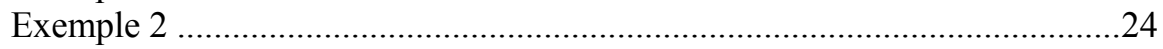

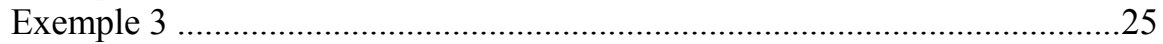

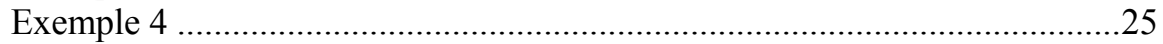

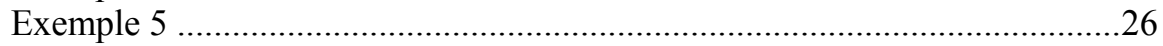

Modifications apportées au chapitre VI des Principes applicables en matière de prix de transfert .........................................................................................31

Chapitre VI. Considérations particulières applicables aux actifs incorporels .....................................................................................................................31

A. Identifier les actifs incorporels........................................................33

[B. Propriété des actifs incorporels et transactions portant sur la mise au point, l'amélioration, l'entretien, la protection et l'exploitation d'actifs incorporels

C. Transactions portant sur l'utilisation ou le transfert d'actifs incorporels

D. Instructions supplémentaires pour déterminer les conditions de pleine concurrence dans le cas de transactions portant sur des actifs incorporels.

Annexe au chapitre VI. Exemples d'application des instructions concernant les considérations particulières applicables aux actifs incorporels

[Exemple 1

Exemple 2 
Exemple 3 ....................................................................................... 113

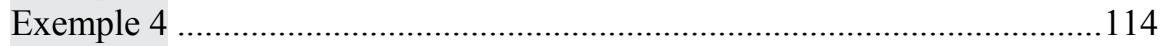

Exemple 5 ................................................................................ 114

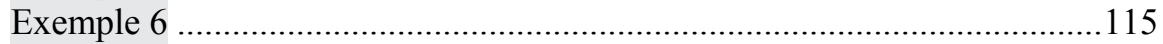

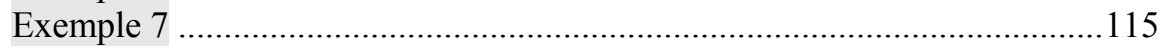

Exemple 8 ............................................................................................. 116

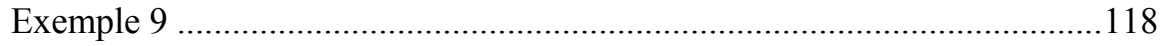

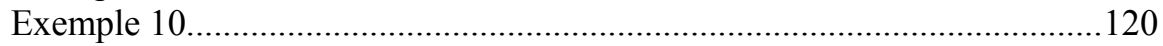

Exemple 11................................................................................... 122

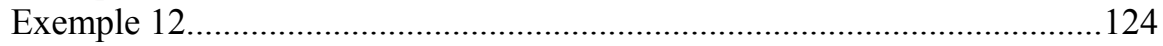

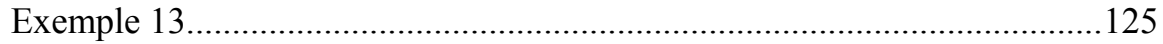

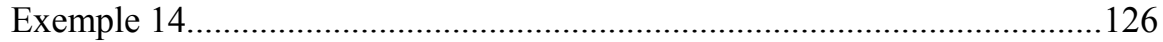

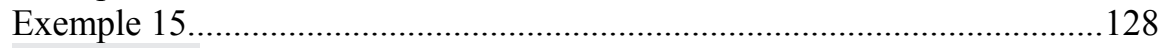

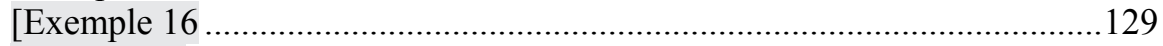

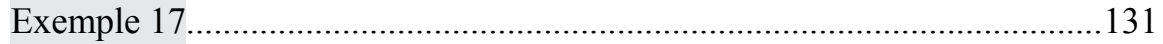

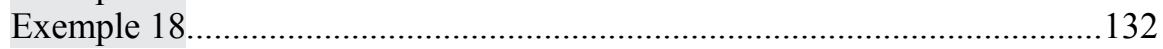

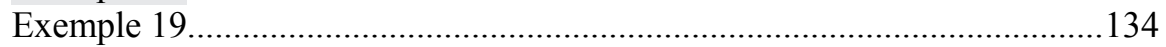

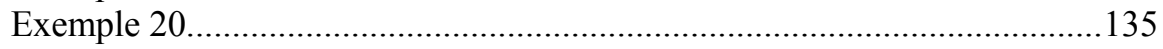

Exemple 21.......................................................................................... 136

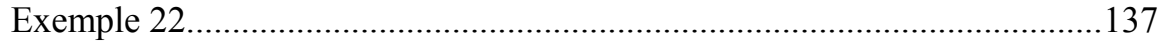

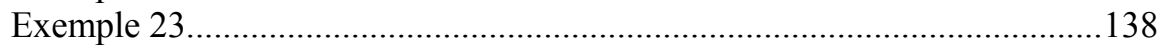

Exemple 24.......................................................................................139

Exemple 25 ............................................................................... 140

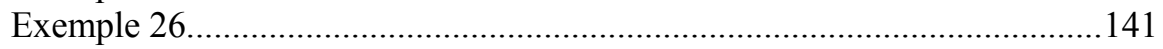

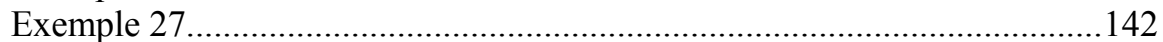

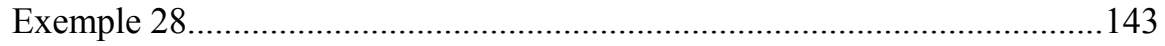

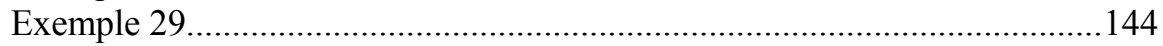

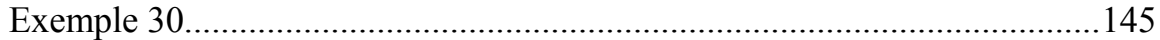

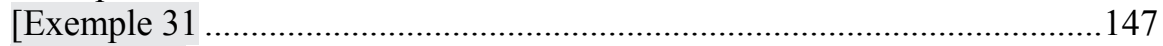

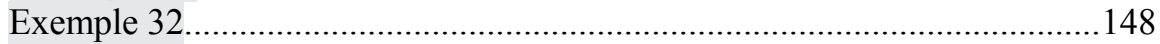

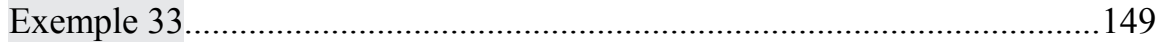




\section{Abréviations et acronymes}

BEPS Érosion de la base d'imposition et transfert de bénéfices (Base erosion and profit shifting)

CAF Comité des affaires fiscales

OCDE Organisation de coopération et de développement économiques

SEC Société étrangère contrôlée

SRC Société de recherche sous contrat 



\section{Synthèse}

Le présent rapport contient des orientations sur les aspects prix de transfert des actifs incorporels. Il présente les révisions finales des chapitres I, II et VI des Principes de l'OCDE applicables en matière de prix de transfert (2010), qui ont été faites dans le cadre de l'action 8 du Plan d'action concernant l'érosion de la base d'imposition et le transfert de bénéfices (OCDE, 2013). Ces changements apportés aux Principes visent à clarifier la définition des actifs incorporels, à fournir des orientations pour l'identification des transactions impliquant des actifs incorporels, et à donner des orientations supplémentaires pour déterminer les conditions de pleine concurrence de ces transactions. Ces révisions finales des Principes contiennent également des instructions sur le traitement des caractéristiques du marché local et des synergies d'entreprise dans l'optique de la détermination des prix de transfert. Ces instructions sont complétées par de nombreux exemples illustrant l'application des prescriptions énoncées dans le texte révisé des Principes.

Les orientations finales figurant dans le présent rapport correspondent à la première phase des travaux sur les prix de transfert requis par le Plan d'action concernant le BEPS.

Selon le Plan d'action, l'OCDE doit examiner plusieurs questions relatives aux prix de transfert, notamment :

\section{Action 8 - Actifs incorporels}

Élaborer des règles qui empêchent l'érosion de la base d'imposition et le transfert de bénéfices par le biais du transfert d'actifs incorporels entre membres d'un même groupe, ce qui implique de prendre les mesures suivantes: (i) adopter une définition large et clairement délimitée des actifs incorporels; (ii) faire en sorte que les bénéfices associés au transfert et à l'utilisation d'actifs incorporels soient correctement répartis en fonction de la création de valeur (et pas indépendamment de cette création de valeur) ; (iii) élaborer des règles de calcul des prix de transfert ou des mesures spéciales applicables aux transferts d'actifs incorporels difficiles à valoriser ; et (iv) mettre à jour les instructions relatives aux accords de répartition des coûts. 


\section{Action 9 - Risques et capital}

Élaborer des règles qui empêchent l'érosion de la base d'imposition et le transfert de bénéfices par le biais du transfert de risques entre membres d'un même groupe ou de l'attribution d'une fraction excessive du capital aux membres de ce groupe. Il faudra pour cela adopter des règles d'établissement des prix de transfert ou des mesures spéciales qui empêchent qu'une entité perçoive des revenus inappropriés du seul fait qu'elle s'est contractuellement engagée à assumer des risques ou à apporter du capital. Les règles à définir devront également imposer que les revenus soient proportionnels à la création de valeur. Ces travaux seront menés en coordination avec ceux relatifs aux déductions des paiements d'intérêts et d'autres frais financiers.

\section{Action 10 - Autres transactions à haut risque}

Élaborer des règles qui empêchent l'érosion de la base d'imposition et le transfert de bénéfices par le biais de transactions dans lesquelles des entreprises indépendantes ne s'engageraient pas, ou ne s'engageraient que rarement. Il faudra pour cela adopter des règles d'établissement des prix de transfert ou des mesures spéciales visant à : (i) préciser les circonstances dans lesquelles des transactions peuvent être requalifiées; (ii) clarifier l'application des méthodes d'établissement des prix de transfert, notamment celles fondées sur le partage des bénéfices, dans le contexte des chaînes de valeur mondiales; et (iii) se prémunir contre les types les plus fréquents de paiements ayant pour effet d'éroder la base d'imposition, comme les frais de gestion et les dépenses du siège.

Selon le Plan d'action, la deuxième phase des travaux sur ces questions doit être achevée en 2015.

Inévitablement, certains aspects prix de transfert des actifs incorporels sont étroitement liés à des questions qui doivent être examinées en 2015 en vertu du Plan d'action. Notamment, de fortes interactions entre les travaux sur la propriété des actifs incorporels au titre de l'action 8 et les travaux sur le risque, la requalification des transactions et les actifs incorporels difficiles à évaluer. En raison de ces interactions, il apparaît difficile de finaliser des orientations dans un domaine sans examiner aussi les autres questions de manière intégrée.

Les interactions existant entre les travaux sur la propriété des actifs incorporels, les actifs incorporels difficiles à valoriser, le risque et la requalification sont particulièrement prononcées, la décision a été prise de ne pas finaliser à ce stade les travaux sur certaines sections de ce rapport. Aussi, les parties entre parenthèses et grisées doivent donc être considérées 
comme des versions provisoires des orientations, pas encore adoptées dans leur intégralité par les délégués, qui seront finalisées en 2015 dans le cadre des autres travaux relatifs à l'érosion de la base d'imposition et au transfert de bénéfices. Les sections contenant des orientations provisoires sont notamment les suivantes: (i) les sections B.1 et B.2 du chapitre VI des Principes applicables en matière de prix de transfert qui concernent la propriété des actifs incorporels; (ii) la section D.3. du chapitre VI concernant les actifs incorporels dont l'évaluation est incertaine au moment de la transaction; (iii) le paragraphe 2.9 des Principes concernant l'utilisation d'autres méthodes; (iv) les orientations sur l'application des méthodes de partage des bénéfices figurant aux paragraphes 6.145 à 6.149 ; et (v) certains exemples concernant les dispositions susvisées.

Les pays participant au projet BEPS prévoient de finaliser ces sections des orientations révisées sur les actifs incorporels en 2015 conjointement à leurs travaux sur le risque, la requalification et les actifs incorporels difficiles à valoriser. Les travaux BEPS à mener en 2015 viseront notamment à réviser certaines parties des chapitres I, II, VI, VIII et IX des Principes et devraient permettre de finaliser les parties entre parenthèses et grisées du présent projet. Pour ce qui est de l'achèvement en 2015 des travaux sur les prix de transfert dans le cadre du projet BEPS, on s'attachera à examiner les questions de manière intégrée afin de produire des orientations cohérentes et compatibles sur les prix de transfert dans les domaines qui impliquent des actifs incorporels et dans les autres domaines.

Pour mener à bien les travaux sur les prix de transfert requis par le Plan d'action BEPS, l'OCDE s'emploiera, comme indiqué dans le Plan d'action, à examiner l'application du principe de pleine concurrence et de mesures spéciales afin de mettre en évidence les moyens de répondre efficacement aux préoccupations soulevées par le Plan d'action. Les discussions sur les mesures spéciales requises pour répondre aux préoccupations mises en évidence dans le Plan d'action sont en cours. Parmi les mesures spéciales qui seront examinées dans le cadre des travaux de 2015 , on peut citer les suivantes :

- Habiliter les administrations fiscales dans des cas appropriés à appliquer les règles fondées sur les résultats effectifs pour déterminer les prix de transfert des actifs incorporels difficiles à évaluer et éventuellement à d'autres actifs ;

- Limiter les revenus attribuables aux entités dont les activités ne concernent que l'apport de financements pour la mise au point d'actifs incorporels, et éventuellement d'autres activités, par exemple 
en traitant ces entités comme des prêteurs et non comme des investisseurs en fonds propres dans certaines circonstances ;

- Exiger des paiements conditionnels et/ou l'application de méthodes de partage des bénéfices pour certains transferts d'actifs incorporels difficiles à évaluer; et

- Exiger l'application de règles analogues à celles appliquées en vertu de l'article 7 et de l'approche autorisée de l'OCDE à certaines situations caractérisées par la capitalisation excessive d'entités n'ayant pas de fonctions importantes.

Les travaux sur ces mesures relatives aux prix de transfert seront coordonnés avec les autres travaux réalisés dans le cadre du projet BEPS concernant la déductibilité des paiements d'intérêts, la définition de l'établissement stable, le régime des sociétés étrangères contrôlées (SEC), l'économie numérique et le règlement des différends. Les délégués pourront ainsi s'attacher à définir un ensemble cohérent de règles qui répondront de manière efficace aux préoccupations concernant les prix de transfert soulevées dans le cadre du BEPS. Il convient de souligner qu'aucune décision n'a encore été prise concernant les mesures spéciales qui seront adoptées ou la question de savoir si ces mesures sont compatibles avec l'article 9 du Modèle de convention fiscale de l'OCDE. La liste susvisée n'est pas nécessairement exhaustive et d'autres mesures pourraient être envisagées.

Étant donné que les sujets couverts par les mesures relatives aux prix de transfert qui figurent dans le Plan d'action concernant le BEPS ont des répercussions évidentes sur les travaux entrepris dans d'autres domaines du projet BEPS, l'OCDE se propose de faire avancer rapidement les travaux sur les risques, la requalification, les actifs incorporels difficiles à évaluer et les mesures spéciales éventuelles. 


\section{Bibliographie}

OCDE (2013), Plan d'action concernant l'érosion de la base d'imposition et le transfert de bénéfices, Éditions OCDE.

http://dx.doi.org/10.1787/9789264203242-fr

OCDE (2010), Principes de l'OCDE applicables en matière de prix de transfert à l'intention des entreprises multinationales et des administrations fiscales 2010, Éditions OCDE.

http://dx.doi.org/10.1787/tpg-2010-fr 



\title{
Modifications apportées aux chapitres I et II des Principes applicables en matière de prix de transfert
}

\author{
Les dispositions suivantes sont insérées dans le chapitre I des Principes \\ applicables en matière de prix de transfert, immédiatement après le \\ paragraphe 1.79 .
}

\section{D.6. Économies de localisation et caractéristiques du marché local}

1.80 Les paragraphes $1.55,1.57$ et 6.31 indiquent que les caractéristiques du marché géographique sur lequel se déroulent les activités industrielles ou commerciales considérées peuvent influer sur la comparabilité et les prix de pleine concurrence. L'évaluation des différences entre marchés géographiques et la détermination des ajustements de comparabilité à effectuer en conséquence peuvent soulever des problèmes épineux. Ceux-ci peuvent se poser dans le contexte de l'examen d'économies imputables à l'exercice d'activités sur un marché donné. Ces économies sont parfois désignées sous le nom d'économies de localisation. Dans d'autres situations, des problèmes de comparabilité peuvent se poser dans le contexte de l'examen d'avantages ou de désavantages liés au marché local qui peuvent n'avoir aucun rapport direct avec les économies de localisation.

\section{D.6.1. Économies de localisation}

1.81 Les paragraphes 9.148 à 9.153 portent sur le traitement des économies de localisation dans le contexte d'une réorganisation d'entreprise. Les principes exposés dans ces paragraphes s'appliquent en général à toutes les situations où il existe des économies de localisation, et non uniquement en cas de réorganisation d'entreprise.

1.82 Suivant les instructions énoncées aux paragraphes 9.148 à 9.153, pour déterminer comment répartir des économies de localisation entre deux entreprises associées ou plus, il est nécessaire de prendre en compte (i) l'existence éventuelle d'économies de localisation ; (ii) le montant de ces éventuelles économies de localisation; (iii) la mesure dans laquelle ces économies de localisation sont soit conservées par un ou plusieurs membres 
du groupe multinational considéré, soit répercutées sur des fournisseurs ou des clients indépendants ; et (iv) lorsque des économies de localisation ne sont pas intégralement répercutées sur des fournisseurs ou des clients indépendants, la façon dont des entreprises indépendantes exerçant leurs activités dans des circonstances similaires répartiraient d'éventuelles économies de localisation nettes ayant été conservées.

1.83 Lorsque l'analyse fonctionnelle montre qu'il existe des économies de localisation qui ne sont pas répercutées sur les clients ni les fournisseurs, et que des transactions et des entités comparables peuvent être identifiées sur le marché local, ces comparables sur le marché local fourniront l'indication la plus fiable quant à la façon dont les économies de localisation nettes devraient être réparties entre deux entreprises associées ou plus. Par conséquent, lorsqu'on dispose de comparables fiables sur le marché local et qu'ils peuvent être utilisés pour calculer des prix des pleine concurrence, des ajustements de comparabilité spécifiques correspondant à des économies de localisation ne devraient pas être nécessaires.

$1.84 \quad$ Faute de comparables fiables sur le marché local, il convient de déterminer l'existence et la répartition d'éventuelles économies de localisation entre les membres d'un groupe multinational, ainsi que les ajustements de comparabilité qui pourraient être nécessaires pour tenir compte de ces économies de localisation, à partir d'une analyse de l'ensemble des faits et circonstances pertinents, notamment des fonctions exercées, des risques assumés et des actifs utilisés par les entreprises associées concernées, suivant les modalités décrites aux paragraphes 9.148 à 9.153 .

\section{D.6.2. Autres caractéristiques du marché local}

1.85 Les caractéristiques du marché local où se déroulent les activités industrielles ou commerciales considérées peuvent influer sur le prix de pleine concurrence s'agissant de transactions entre entreprises associées. Si certaines caractéristiques peuvent se traduire par des économies de localisation, d'autres peuvent soulever des problèmes de comparabilité sans rapport direct avec ces économies. Ainsi, l'analyse de comparabilité, notamment fonctionnelle, réalisée en lien avec un cas particulier peut indiquer que les caractéristiques pertinentes du marché géographique sur lequel les produits sont fabriqués ou vendus, le pouvoir d'achat et les préférences des ménages en termes de produits sur ce marché, le fait que ce marché se développe ou se contracte, l'intensité de la concurrence sur ce marché et d'autres facteurs similaires influent sur les prix et les marges pouvant être réalisés sur le marché considéré. De même, l'analyse de comparabilité, notamment fonctionnelle, réalisée en lien avec un cas 
particulier peut indiquer que la disponibilité relative d'infrastructures locales dans le pays considéré, la disponibilité relative d'un réservoir de maind'œuvre qualifiée, la proximité de marchés rentables et des caractéristiques similaires sur un marché géographique où se déroulent les activités industrielles ou commerciales considérées créent des avantages ou des désavantages liés au marché qui doivent être pris en considération. Des ajustements de comparabilité appropriés doivent être effectués pour tenir compte de ces facteurs dès lors qu'il est possible d'identifier des ajustements fiables permettant d'améliorer la comparabilité.

1.86 Pour déterminer si des ajustements de comparabilité sont nécessaires concernant ce type de caractéristiques d'un marché local, l'approche la plus fiable consistera à s'appuyer sur des données concernant des transactions comparables sur le marché libre ayant lieu sur ce marché géographique entre des entreprises indépendantes qui exercent des fonctions similaires, assument des risques similaires et utilisent des actifs similaires. Ces transactions sont réalisées dans les mêmes conditions de marché que la transaction contrôlée et, en conséquence, dès lors que des transactions comparables peuvent être identifiées sur le marché local, aucun ajustement spécifique ne devrait être nécessaire pour tenir compte des caractéristiques du marché local.

1.87 Dans les situations où il est impossible d'identifier sur le marché local des comparables raisonnablement fiables, les ajustements de comparabilité appropriés pour tenir compte des caractéristiques du marché local doivent être déterminés sur la base de l'ensemble des faits et circonstances pertinents. Comme pour les économies de localisation, dès lors qu'il est impossible d'identifier des comparables fiables sur le marché local, il est nécessaire de prendre en compte (i) l'existence éventuelle d'un avantage ou d'un désavantage lié au marché, (ii) le montant de toute augmentation ou diminution du chiffre d'affaires, des coûts ou des bénéfices, par rapport à des comparables identifiés sur d'autres marchés, imputable à l'avantage ou désavantage lié au marché local, (iii) la mesure dans laquelle les avantages ou les charges liés aux caractéristiques du marché local sont répercutés sur des fournisseurs ou des clients indépendants, et (iv) lorsqu'il existe des avantages ou des charges liés aux caractéristiques du marché local qui ne sont pas intégralement répercutés sur des fournisseurs ou des clients indépendants, la façon dont des entreprises indépendantes exerçant leurs activités dans des circonstances similaires répartiraient ces avantages ou charges nets entre elles.

1.88 La nécessité de procéder à des ajustements de comparabilité liés aux caractéristiques du marché local lorsqu'il est impossible d'identifier sur 
le marché local des comparables raisonnablement fiables peut se présenter dans différents contextes. Dans certaines circonstances, des avantages ou des désavantages liés au marché peuvent influer sur le prix de pleine concurrence de biens transférés ou de services fournis entre des entreprises associées.

1.89 Dans d'autres circonstances, une réorganisation d'entreprise ou le transfert d'actifs incorporels entre des entreprises associées peut permettre à une partie à la transaction considérée de bénéficier d'avantages liés au marché local, ou imposer à cette partie le fardeau de désavantages liés au marché local d'une façon qui n'aurait pas été possible en l'absence de cette réorganisation d'entreprise ou de ce transfert d'actifs incorporels. Dans ces circonstances, l'existence anticipée d'avantages ou de désavantages liés au marché local peut influer sur le prix de pleine concurrence acquitté en relation avec la réorganisation d'entreprise ou le transfert d'actifs incorporels considéré.

1.90 Lors de la réalisation d'une analyse de prix de transfert, il importe de faire la distinction entre les caractéristiques du marché local, qui ne sont pas des actifs incorporels, et les éventuels droits contractuels, permis ou savoir-faire nécessaires pour exploiter ce marché, qui peuvent être des actifs incorporels. Suivant les circonstances, ces types d'actifs incorporels peuvent avoir une valeur substantielle, qui doit être prise en compte dans le cadre d'une analyse des prix de transfert suivant les modalités décrites au chapitre VI, notamment les instructions sur la rémunération des entités qui exercent des fonctions, utilisent des actifs et assument des risques liés à la mise au point d'actifs incorporels qui figurent dans la section B du chapitre VI. Dans certaines circonstances, des droits contractuels et des permis peuvent limiter l'accès des concurrents à un marché donné et peuvent par conséquent influer sur la façon dont les retombées économiques des caractéristiques du marché local sont réparties entre les parties à une transaction donnée. Dans d'autres circonstances, les droits contractuels ou les permis nécessaires pour entrer sur un marché peuvent être accessibles à de nombreux ou à tous les entrants potentiels sur ce marché avec des restrictions limitées.

1.91 Ainsi, un pays peut exiger la délivrance d'un permis réglementaire à titre de préalable à l'exercice d'activités de gestion de placements dans ledit pays, et peut limiter le nombre d'entreprises à capitaux étrangers auxquelles ce type de permis est octroyé. L'analyse de comparabilité, notamment fonctionnelle, peut indiquer que pour obtenir un tel permis, il faut démontrer aux autorités compétentes que le fournisseur de services dispose d'une expérience et d'un capital suffisants pour pouvoir 
exercer les activités en question de manière sérieuse. Le marché auquel s'applique ce type de permis peut également présenter des caractéristiques uniques. Il peut s'agir, par exemple, d'un marché où la structure des dispositifs de retraite et d'assurance se traduit par d'amples réserves de liquidités, un besoin de diversification internationale des placements et, par voie de conséquence, une forte demande de services de gestion de placements de qualité et de connaissance des marchés de capitaux étrangers de nature à rendre ces prestations de services extrêmement lucratives. L'analyse de comparabilité peut également indiquer que ces caractéristiques du marché local peuvent influer sur le prix pouvant être facturé pour certains types de services de gestion de placements, et sur les marges pouvant être réalisées sur ces prestations de services. Dans ces circonstances, l'actif incorporel considéré (à savoir le permis réglementaire nécessaire pour fournir des services de gestion de placements) peut permettre à la partie ou aux parties détenant le permis d'obtenir une plus grande part des avantages qui découlent des activités exercées sur le marché local, notamment des avantages inhérents aux caractéristiques uniques de ce marché, que celle qui serait obtenue en l'absence de l'obligation de détention du permis. Néanmoins, pour évaluer l'impact de ce permis réglementaire, il peut être important dans un cas particulier d'examiner les contributions du membre local du groupe considéré sur le marché local ainsi que des autres membres du groupe en dehors du marché local à l'apport des moyens nécessaires pour obtenir le permis, comme indiqué à la section B du chapitre VI.

1.92 Dans des circonstances différentes, l'analyse de comparabilité, notamment fonctionnelle, peut indiquer qu'un permis d'exercer une activité économique accordé par les pouvoirs publics constitue un préalable à la fourniture d'un service particulier sur un marché géographique donné. Néanmoins, il peut s'avérer que ces permis sont aisément accessibles à tout demandeur remplissant les conditions requises, et qu'elles n'ont pas pour effet de restreindre le nombre de concurrents présents sur le marché. Dans ces circonstances, l'obligation d'obtention d'un permis peut ne pas constituer un obstacle important à l'entrée, et la détention d'un tel permis peut ne pas avoir d'impact perceptible sur la façon dont les avantages découlant de l'exercice d'activités sur le marché local sont répartis entre des entreprises indépendantes.

\section{D.7. Collectif de travail}

1.93 Certaines entreprises réussissent à constituer un ensemble d'employés caractérisé par des qualifications et une expérience uniques. L'existence d'un tel groupe d'employés peut influer sur le prix de pleine concurrence des services fournis par ce groupe d'employés, ou sur 
l'efficacité avec laquelle l'entreprise considérée fournit des services ou produits des biens. Ces facteurs doivent normalement être pris en compte dans le cadre d'une analyse de comparabilité axée sur les prix de transfert. Lorsqu'il est possible de cerner les avantages ou les inconvénients que représente un collectif de travail unique par rapport au personnel d'entreprises se livrant à des transactions potentiellement comparables, des ajustements de comparabilité peuvent être effectués pour tenir compte de l'impact de ce collectif de travail sur les prix de pleine concurrence de biens ou services.

1.94 Lors de certaines opérations de réorganisation d'entreprise et transactions similaires, il peut arriver qu'un collectif de travail soit transféré d'une entreprise associée à une autre dans le cadre de l'opération considérée. Dans ces circonstances, il est tout à fait possible que le transfert de ce collectif de travail, ainsi que d'autres actifs de l'entreprise en question, permette au cessionnaire d'économiser le temps et les ressources financières nécessaires à l'embauche et à la formation d'une nouvelle main-d'œuvre. Suivant les méthodes d'établissement des prix de transfert utilisées pour évaluer la transaction dans son ensemble, il peut être approprié dans ce cas de figure de prendre en compte ces économies de temps et de ressources financières sous la forme d'ajustements de comparabilité apportés au prix de pleine concurrence qui serait appliqué en leur absence aux actifs transférés. Dans d'autres situations, le transfert du collectif de travail peut limiter la marge de manœuvre dont dispose le cessionnaire pour organiser les activités industrielles ou commerciales considérées et peut créer des éléments de passif éventuel en cas de licenciements. Dans ce cas, il peut être approprié que la rémunération versée au titre de la réorganisation reflète les éventuels restrictions et éléments de passif futurs.

1.95 Le paragraphe qui précède n'implique aucunement que chaque transfert ou détachement d'employé entre membres d'un groupe multinational doit faire l'objet d'une rémunération distincte en règle générale. Dans de nombreux cas, le transfert d'un employé entre des entreprises associées ne donnera pas nécessairement lieu à une rémunération. Lorsque des employés sont détachés (ce qui signifie qu'ils demeurent salariés du cédant mais travaillent pour le cessionnaire), la rémunération de pleine concurrence appropriée des services fournis par ces employés détachés sera dans de nombreux cas le seul paiement requis.

1.96 Il convient cependant de noter que, dans certaines situations, le transfert ou le détachement d'un ou plusieurs employés peut, en fonction des faits et circonstances, se traduire par le transfert d'un savoir-faire précieux ou d'autres actifs incorporels de valeur d'une entreprise associée à une autre. 
Ainsi, un employé de la société $\mathrm{A}$ détaché auprès de la société $\mathrm{B}$ peut avoir connaissance d'une formule secrète appartenant à la société $\mathrm{A}$, et peut la mettre à la disposition de la société $\mathrm{B}$ pour qu'elle en fasse usage dans le cadre de ses activités commerciales. De même, des employés de la société A détachés auprès de la société $B$ pour contribuer au démarrage d'une usine peuvent mettre le savoir-faire productif de la société $\mathrm{A}$ à la disposition de la société B pour qu'elle en fasse usage dans le cadre de ses activités commerciales. Lorsque la fourniture d'un tel savoir-faire ou d'autres actifs incorporels résulte du transfert ou du détachement d'employés, elle devrait faire l'objet d'une analyse distincte en vertu des dispositions du chapitre VI, et un prix approprié devrait être payé pour le droit d'utiliser les actifs incorporels en question.

1.97 Par ailleurs, il convient également de noter que l'accès à un collectif de travail doté d'une expérience et de qualifications particulières peut, dans certaines circonstances, accroître la valeur des actifs incorporels ou autres transférés, même si les employés constituant ce personnel ne sont pas transférés. L'exemple 24 qui figure dans l'annexe au chapitre VI illustre un scénario dans lequel l'interaction entre les actifs incorporels et l'accès à un collectif de travail peuvent être important dans le cadre d'une analyse de prix de transfert.

\section{D.8. Synergies de groupe multinational}

1.98 Des problèmes de comparabilité, et la nécessité d'ajustements de comparabilité, peuvent également découler de l'existence de synergies de groupe multinational. Dans certaines circonstances, un groupe multinational et les entreprises associées qui le constituent peuvent tirer parti d'interactions ou de synergies entre les membres de ce groupe qui n'existeraient généralement pas entre des entreprises indépendantes se trouvant dans une situation similaire. Ces synergies de groupe peuvent notamment découler, par exemple, d'une puissance d'achat cumulée ou d'économies d'échelle, de la conjonction et de l'intégration des systèmes informatiques et de communication, d'un système de gestion intégré, de l'élimination de doubles emplois, d'une capacité d'emprunt accrue, ainsi que de nombreux autres facteurs similaires. De telles synergies de groupe sont souvent favorables à l'ensemble du groupe considéré et peuvent donc se traduire par une augmentation du montant global des bénéfices engrangés par les membres du groupe, selon que les économies attendues sont effectivement réalisées ou non et suivant les conditions de concurrence. Dans d'autres circonstances, de telles synergies peuvent être négatives, notamment lorsque l'ampleur et le champ des activités du groupe créent des obstacles bureaucratiques auxquels ne sont pas confrontés des entreprises plus petites et plus réactives, ou 
lorsqu'une partie du groupe est contrainte de fonctionner avec des systèmes informatiques ou de communication qui ne sont pas optimaux au regard de ses activités en raison de normes définies pour l'ensemble du groupe multinational.

1.99 Selon le paragraphe 7.13 de ces Principes, il ne faut pas considérer qu'une entreprise associée bénéficie d'un service intra-groupe, ni lui imposer un paiement, lorsqu'elle obtient des avantages accessoires qui sont uniquement imputables au fait qu'elle fait partie d'un groupe multinational plus vaste. Dans ce contexte, le terme «accessoire» désigne des avantages découlant uniquement de l'affiliation à un groupe, obtenus en l'absence de toute transaction ou action délibérée et concertée débouchant sur ces avantages. Le terme «accessoire» ne fait aucunement référence à l'ampleur de ces avantages et n'implique en aucun cas que ces avantages doivent être modestes ou relativement peu importants. En accord avec cette vision générale des avantages accessoires découlant de l'appartenance à un groupe, lorsque des avantages ou des inconvénients synergiques découlent uniquement de l'appartenance à un groupe multinational, sans la moindre action délibérée et concertée de membres de ce groupe et en l'absence de prestation de services ou d'exercice d'autres fonctions par des membres de groupe, il n'est pas nécessaire que ces avantages synergiques résultant de l'appartenance au groupe considéré donnent lieu à une rémunération distincte, ni qu'ils fassent l'objet d'une répartition spécifique entre les membres du groupe multinational en question.

1.100 Dans certaines circonstances, néanmoins, des avantages et inconvénients synergiques résultant de l'appartenance à un groupe peuvent découler d'actions délibérées et concertées de la part de ce groupe multinational, et peuvent lui conférer un avantage ou un désavantage structurel important, clairement identifiable sur le marché considéré, par rapport aux participants qui n'appartiennent pas à un groupe multinational et qui se livrent à des transactions comparables. Seule une analyse de comparabilité, notamment fonctionnelle, approfondie peut permettre de déterminer si un tel avantage ou désavantage structurel existe, quelle peut être la nature et la source de l'avantage ou de l'inconvénient synergique considéré, et si cet avantage ou cet inconvénient synergique résulte d'actions délibérées et concertées de la part du groupe concerné ${ }^{1}$.

1.101 Ainsi, si un groupe prend délibérément des mesures pour centraliser les achats au sein d'une seule société du groupe afin d'obtenir des rabais liés au volume fourni, et si cette société revend les articles qu'elle achète aux autres membres du groupe, on est en présence d'une action délibérée et concertée de la part du groupe destinée à tirer parti de sa 
puissance d'achat. De même, si directeur d'achat central en poste à la société mère ou dans un centre de gestion régional fournit un service en négociant avec un fournisseur un rabais pour l'ensemble du groupe subordonné à un niveau d'achats minimum à l'échelle du groupe, et si des membres du groupe effectuent ensuite des achats auprès de ce fournisseur et obtiennent ce rabais, on est en présence d'une action délibérée et concertée de la part du groupe, nonobstant l'absence d'opérations spécifiques d'achat et de vente entre les membres du groupe considéré. En revanche, lorsqu'un fournisseur offre unilatéralement à un membre du groupe un prix favorable dans l'espoir d'élargir le champ de ses relations commerciales à d'autres membres du groupe, il n'y a pas d'action délibérée et concertée de la part du groupe.

1.102 Lorsque des synergies d'entreprise découlant d'actions délibérées et concertées de la part d'un groupe sont effectivement la source pour un membre de ce groupe multinational d'avantages ou d'inconvénients importants rares chez les entreprises indépendantes comparables, il est nécessaire de déterminer (i) la nature de l'avantage ou du désavantage considéré, (ii) le montant que représente cet avantage ou inconvénient et (iii) la façon dont cet avantage ou inconvénient doit être réparti entre les membres du groupe multinational.

1.103 S'il existe d'importantes synergies de groupe pouvant être attribuées à des actions délibérées et concertées de la part du groupe considéré, les avantages découlant de ces synergies doivent généralement être partagés par les membres du groupe à proportion de leur contribution à la création de la synergie considérée. Ainsi, lorsque des membres du groupe engagent des actions délibérées et concertées pour regrouper des activités d'achat en vue de tirer parti des économies d'échelle résultant de gros volumes d'achats, les avantages découlant de ces synergies liées à des achats massifs, s'il en existe après rémunération appropriée de la partie coordonnant les activités d'achat, doivent généralement être partagés par les membres du groupe à proportion de leur volume d'achats.

1.104 Des ajustements de comparabilité peuvent être nécessaires pour tenir compte des synergies de groupe.

\section{Exemple 1}

$1.105 \quad \mathrm{P}$ est la société mère d'un groupe multinational exerçant des activités de services financiers. Grâce à la solidité du bilan consolidé du groupe, $\mathrm{P}$ se voit régulièrement attribuer la notation financière AAA. S est un membre d'un groupe multinational qui fournit le même type de services financiers que d'autres membres du groupe et le fait à grande échelle sur un 
marché important. Considérée isolément, toutefois, la solidité du bilan de $\mathrm{S}$ justifierait une notation de Baa seulement. Néanmoins, du fait de l'appartenance de $\mathrm{S}$ au groupe $\mathrm{P}$, des grands prêteurs indépendants sont disposés à lui prêter de l'argent à des taux d'intérêt qui seraient appliqués à des emprunteurs indépendants notés $\mathrm{A}$, c'est-à-dire un taux d'intérêt inférieur à celui qui serait appliqué à $S$ s'il s'agissait d'une entité indépendante ayant le même bilan, mais un taux d'intérêt plus élevé que celui dont bénéficierait la société mère du groupe multinational.

1.106 Supposons que $\mathrm{S}$ emprunte 50 millions EUR à un prêteur indépendant au taux d'intérêt du marché appliqué aux emprunteurs notés A. Supposons par ailleurs que $\mathrm{S}$ emprunte simultanément 50 millions EUR à $\mathrm{T}$, une autre filiale de $\mathrm{P}$, présentant des caractéristiques similaires à celles du prêteur indépendant, aux mêmes conditions et au même taux d'intérêt que ceux appliqués par le prêteur indépendant (à savoir un taux d'intérêt fondé sur l'existence d'une notation A). Supposons en outre que le prêteur indépendant, lors de la fixation de ces conditions, avait connaissance des autres emprunts de $\mathrm{S}$, notamment du prêt accordé simultanément à $\mathrm{S}$ par $\mathrm{T}$.

1.107 Dans ces circonstances, le taux d'intérêt appliqué au prêt accordé par $\mathrm{T}$ à $\mathrm{S}$ est un taux de pleine concurrence parce que (i) c'est le même taux qui est appliqué à $\mathrm{S}$ par un prêteur indépendant dans le cadre d'une transaction comparable; et (ii) aucun paiement ou ajustement de comparabilité n'est requis pour l'avantage synergique de groupe qui permet à $\mathrm{S}$ d'emprunter auprès d'une entreprise indépendante à un taux d'intérêt plus faible qu'elle ne le pourrait si elle n'était pas membre du groupe considéré, parce que l'avantage synergique représenté par cette capacité d'emprunt trouve uniquement son origine dans l'appartenance de $\mathrm{S}$ à ce groupe, et n'est aucunement imputable à une action délibérée et concertée de la part de membres du groupe multinational en question.

\section{Exemple $2^{2}$}

1.108 La situation de $\mathrm{S}$ en termes de notation et de capacité d'emprunt est identique à celle de l'exemple précédent. $\mathrm{S}$ emprunte 50 millions EUR à la banque $\mathrm{A}$. L'analyse fonctionnelle indique que la banque $\mathrm{A}$ prêterait à $\mathrm{S}$ à un taux d'intérêt applicable aux emprunteurs notés A sans aucune garantie en bonne et due forme. Néanmoins, la société mère accepte de garantir le prêt de la banque $\mathrm{A}$ afin d'inciter celle-ci à prêter au taux d'intérêt dont bénéficieraient des emprunteurs notés AAA. Dans ces circonstances, S doit être tenue de payer une commission de garantie à la société mère au titre de cette garantie expresse. Le calcul d'une commission de garantie de pleine concurrence doit se fonder sur l'avantage conféré par un relèvement de la 
notation de $\mathrm{S}$ de $\mathrm{A}$ à $\mathrm{AAA}$, et non sur l'avantage conféré par un relèvement de cette notation de Baa à AAA. Le rehaussement de la notation de $\mathrm{S}$ de Baa à $\mathrm{A}$ est en effet imputable à la synergie de groupe découlant exclusivement d'une association passive au groupe, qui n'a pas à être rémunérée en vertu des dispositions de la présente section. Le relèvement de la notation de $\mathrm{S}$ de A à AAA, en revanche, est imputable à une action délibérée et concertée, à savoir la fourniture d'une garantie par la société mère, et doit donc donner lieu à une rémunération.

\section{Exemple 3}

1.109 Supposons que la société A se voie confier le rôle de directeur d'achats central pour l'ensemble du groupe considéré. Elle achète des produits à des fournisseurs indépendants et les revend aux entreprises associées. La société $\mathrm{A}$, uniquement grâce au pouvoir de négociation conféré par la puissance d'achat de l'ensemble du groupe, est en mesure de négocier avec un fournisseur pour faire baisser le prix de certains articles de 200 USD à 110 USD. Dans ces circonstances, le prix de pleine concurrence pour la revente de ces articles par la société A aux autres membres du groupe n'est pas égal ou proche de 200 USD. Ce prix de pleine concurrence correspond au montant permettant de rémunérer la société A pour ses services de coordination des activités d'achat. Si l'analyse de comparabilité, notamment fonctionnelle, indique dans ce cas que, dans le cadre de transactions comparables sur le marché libre portant sur un volume d'achats comparable, des services de coordination comparables donnent lieu au versement d'une commission correspondant aux coûts assumés par la société A augmentés d'une marge représentant une rémunération totale de 6 USD par article, le prix de revente de ces articles par la société A aux autres entreprises du groupe sera approximativement de 116 USD. Dans ces circonstances, chaque membre du groupe obtiendra un avantage imputable à la puissance d'achat du groupe de l'ordre de 84 USD par article. En outre, la société A gagnera 6 USD par article acheté par des membres du groupe au titre de ses prestations de services.

\section{Exemple 4}

1.110 Posons l'hypothèse d'une situation similaire à celle décrite dans l'exemple 3, hormis le fait qu'au lieu d'acheter effectivement puis de revendre les articles considérés, la société A négocie un rabais au nom du groupe et les membres du groupe achètent ensuite directement ces articles au fournisseur indépendant. Dans ces circonstances, supposons que l'analyse de comparabilité indique que la société A est en droit de percevoir une commission de 5 USD par article au titre des services de coordination 
fournis aux autres membres du groupe (le niveau plus faible de la commission retenu comme hypothèse dans l'exemple 4, par rapport à l'exemple 3 , peut correspondre à un moindre niveau de risque assumé par le fournisseur de service compte tenu du fait qu'il ne devient pas propriétaire des articles et ne détient aucun stock). Les membres du groupe achetant des articles conservent le bénéfice de la remise accordée au groupe pour leurs propres achats, après paiement d'une commission.

\section{Exemple 5}

1.111 Supposons qu'un groupe multinational domicilié dans le pays A ait des filiales manufacturières dans le pays $\mathrm{B}$ et le pays $\mathrm{C}$. Le pays B se caractérise par un taux d'imposition de 30 pour cent et le pays $C$ par un taux d'imposition de 10 pour cent. Le groupe possède également un centre de services partagés dans le pays $\mathrm{D}$. Supposons que les filiales manufacturières situées dans les pays $\mathrm{B}$ et $\mathrm{C}$ ont chacune besoin de 5000 articles produits par un fournisseur indépendant, destinés à servir de consommation intermédiaire dans leur processus de production. Supposons par ailleurs que le centre de services partagés situé dans le pays $\mathrm{D}$ est rémunéré de manière systématique pour l'ensemble de ses activités par les autres membres du groupe, dont les filiales manufacturières situées dans les pays $\mathrm{B}$ et $\mathrm{C}$, selon la méthode du coût majoré, et que cela se traduit, dans le contexte de cet exemple, par une rémunération de pleine concurrence correspondant au volume et à la nature des services qu'il fournit.

1.112 Le fournisseur indépendant vend ses articles 10 USD pièce et applique une politique consistant à accorder une remise de 5 pour cent si la quantité d'articles achetés est supérieure à 7500 articles. Un employé chargé des achats au centre de services partagés dans le pays $\mathrm{D}$ prend contact avec ce fournisseur indépendant et obtient confirmation du fait que, si les filiales manufacturières des pays $\mathrm{B}$ et $\mathrm{C}$ achètent simultanément 5000 articles chacun, soit un achat total de 10000 articles à l'échelle du groupe, la remise sera appliquée à l'ensemble des achats du groupe. Le fournisseur indépendant confirme qu'il vendra au total 10000 articles au groupe multinational considéré pour un prix total de $95000 \mathrm{USD}$, ce qui représente une remise de 5 pour cent par rapport au prix auquel l'une ou l'autre des deux filiales manufacturières pourrait acheter seule ces marchandises à ce fournisseur.

1.113 Cet employé chargé des achats au centre de services partagés passe ensuite commande des articles requis et demande que le fournisseur facture à la filiale manufacturière située dans le pays B 5000 articles au prix total de 50000 USD, et qu'il facture à la filiale manufacturière située dans le 
pays C 5000 articles au prix total de 45000 USD. Le fournisseur accède à cette demande, qui se traduira pour lui par le paiement du prix convenu, à savoir 95000 USD, pour l'ensemble des 10000 articles livrés.

1.114 Dans ces circonstances, le pays $\mathrm{B}$ est en droit de procéder à un ajustement de prix de transfert consistant à réduire de 2500 USD les dépenses de la filiale manufacturière située dans le pays $\mathrm{B}$. Cet ajustement de prix de transfert est approprié, car l'accord conclu en matière de prix fausse la répartition de l'avantage découlant de la synergie de groupe associée à l'acquisition d'articles en grande quantité. Cet ajustement est approprié nonobstant le fait que la filiale manufacturière située dans le pays $\mathrm{B}$ ne pourrait, seule, acheter les articles considérés à un prix inférieur aux 50000 USD qui lui sont facturés. L'action délibérée et concertée de la part du groupe ayant permis l'obtention de la remise est la base sur laquelle se fonde l'affectation d'une partie de cette remise à la filiale manufacturière située dans le pays $\mathrm{B}$, nonobstant l'absence de transaction explicite entre les filiales manufacturières situées dans les pays $B$ et $C$. 
Le paragraphe 2.9 ci-après est reproduit tel qu'il figure dans la version de 2010 des Principes de l'OCDE applicables en matière de prix de transfert. Cette version du paragraphe 2.9 sera révisée et mise à jour dans le cadre des travaux de $\mathbf{2 0 1 5}$ sur les prix de transfert concernant le BEPS. Les modifications apportées prendront en compte les instructions concernant l'application des techniques d'évaluation décrites dans la section D du chapitre VI et au chapitre IX. Il s'agira également de déterminer s'il faut modifier le paragraphe 2.9 pour tenir compte d'autres instructions sur les méthodes d'établissement des prix de transfert et/ou des mesures spéciales qui pourraient être adoptées en lien avec les travaux de 2015 sur le BEPS.

2.9 [Par ailleurs, les groupes multinationaux sont entièrement libres de recourir à des méthodes autres que celles qui sont exposées dans ces Principes (ci-après dénommées «autres méthodes »), dès lors que les prix fixés satisfont au principe de pleine concurrence, conformément à ces Principes. Toutefois, ces autres méthodes ne doivent pas se substituer à celles reconnues par l'OCDE lorsque ces dernières sont plus appropriées aux faits et circonstances du cas d'espèce. Lorsque d'autres méthodes sont utilisées, leur sélection devrait être étayée par une explication des raisons pour lesquelles les méthodes reconnues par l'OCDE ont été considérées comme moins appropriées ou non applicables au cas d'espèce, ainsi que de la raison pour laquelle on a considéré qu'une autre méthode donnait une meilleure solution. Le contribuable devrait conserver une documentation concernant la façon dont ses prix de transfert ont été établis et être prêt à la communiquer. À propos de la documentation, voir le chapitre V.]

Les dispositions suivantes sont insérées à la suite du paragraphe 2.9 et les paragraphes du chapitre II consécutifs au paragraphe 2.9 sont renumérotés en conséquence.

2.10 L'application d'une règle empirique générale ne constitue pas un substitut adéquat à une analyse de comparabilité, notamment fonctionnelle, complète réalisée suivant les principes énoncés aux chapitres I à III. En conséquence, une règle empirique ne peut être utilisée pour démontrer qu'un prix ou une imputation de revenus est conforme au principe de pleine concurrence. 


\section{Notes}

1. Compte tenu des différences entre législations locales, certains pays considèrent qu'une action délibérée et concertée constitue toujours une transaction, d'autres non. Néanmoins, le consensus qui prévaut est que, dans chacun de ces deux cas de figure, il y a action délibérée et concertée dès lors qu'une entreprise associée exerce des fonctions, utilise des actifs ou assume des risques au bénéfice d'une ou plusieurs autres entreprises associées de telle sorte qu'une rémunération de pleine concurrence s'impose. Voir notamment l'exemple 5 ci-après.

2. L'action 4 du Plan d'action concernant l'érosion de la base d'imposition et le transfert de bénéfices (BEPS) prévoit des travaux complémentaires sur les aspects des transactions financières touchant aux prix de transfert. On ne saurait considérer que l'exemple 2 offre des instructions exhaustives concernant les commissions de garantie dans le contexte des prix de transfert. Dans le cadre du projet BEPS sera examinée de manière plus poussée la question de la détermination des conditions de pleine concurrence pour les garanties, en particulier dans les situations où des garanties accordées entre entreprises associées peuvent se traduire par des phénomènes d'érosion de la base d'imposition et de transfert de bénéfices. 



\title{
Modifications apportées au chapitre VI des Principes applicables en matière de prix de transfert
}

\author{
Les dispositions actuelles du chapitre VI des Principes applicables en \\ matière de prix de transfert sont supprimées dans leur intégralité, et \\ remplacées par celles qui suivent.
}

\section{Chapitre VI}

\section{Considérations particulières applicables aux actifs incorporels}

6.1 Aux termes de l'article 9 du Modèle de Convention fiscale de l'OCDE, lorsque les conditions convenues ou imposées pour l'utilisation ou le transfert d'actifs incorporels entre deux entreprises associées ne sont pas les mêmes que celles qui auraient été convenues entre des entreprises indépendantes, les bénéfices qui, sans ces conditions, auraient été réalisés par l'une des entreprises mais n'ont pu l'être du fait de ces conditions, peuvent être inclus dans les bénéfices de cette entreprise et imposés en conséquence.

6.2 L'objet du présent chapitre VI est de fournir des instructions spécifiques en vue de la détermination des conditions de pleine concurrence pour les transactions qui portent sur l'utilisation ou le transfert d'actifs incorporels. L'article 9 du Modèle de Convention fiscale de l'OCDE a trait aux conditions applicables aux transactions entre entreprises associées, et ne vise pas à classer ces opérations dans telle ou telle catégorie. Par conséquent, la considération essentielle est de déterminer si une transaction se traduit par un transfert de valeur économique d'une entreprise associée à 
une autre, si cet avantage découle de biens corporels, d'actifs incorporels, de services ou d'autres éléments ou activités. Un élément ou une activité peut avoir une valeur économique même s'il n'est pas expressément mentionné au chapitre VI. Dès lors qu'un élément ou une activité a une valeur économique, il doit être pris en compte pour déterminer les prix de pleine concurrence, qu'il constitue ou non un actif incorporel au sens du paragraphe 6.6.

6.3 Les prescriptions énoncées aux chapitres I à III de ces Principes s'appliquent aussi bien aux transactions portant sur des actifs incorporels qu'aux autres transactions. Selon ces prescriptions, comme pour d'autres questions relatives aux prix de transfert, l'analyse des cas d'utilisation ou de transfert d'actifs incorporels doit débuter par une analyse de comparabilité approfondie, notamment une analyse fonctionnelle. Cette analyse fonctionnelle doit permettre de cerner les fonctions exercées, les actifs utilisés et les risques assumés par chaque membre du groupe multinational concerné. Dans les cas d'utilisation ou de transfert d'actifs incorporels, il est particulièrement important de fonder l'analyse de comparabilité, notamment fonctionnelle, sur une compréhension des activités du groupe multinational considéré à l'échelle mondiale, et de la façon dont les actifs incorporels sont utilisés par ce groupe multinational pour ajouter ou créer de la valeur tout au long de la chaîne d'approvisionnement. Si nécessaire, il faut se demander lors de l'analyse effectuée, dans le cadre des paragraphes 1.64 à 1.69, si des parties indépendantes auraient conclu l'accord considéré et, si oui, quelles en auraient été les modalités.

6.4 Afin de déterminer les conditions de pleine concurrence relatives à l'utilisation ou au transfert d'actifs incorporels, il importe de prendre en compte dans le cadre de l'analyse de comparabilité, notamment fonctionnelle : (i) l'identification des actifs incorporels spécifiques; (ii) la propriété légale des actifs incorporels ; (iii) les contributions des membres du groupe multinational considéré à leur mise au point, leur amélioration, leur entretien, leur protection et leur exploitation; et (iv) la nature des transactions contrôlées portant sur des actifs incorporels, y compris la façon dont ces transactions contribuent à la création de valeur. Sur cette base, il est ensuite nécessaire d'examiner la rémunération qui serait versée entre parties indépendantes lors de transactions portant sur des actifs incorporels. Ces questions sont traitées dans les sections suivantes. 


\section{A. Identifier les actifs incorporels}

\section{A.1. Généralités}

6.5 Une définition du terme « incorporel» trop étroite ou trop large peut poser problème dans le cadre d'une analyse de prix de transfert. Si l'on applique une définition trop étroite du terme «incorporel», les contribuables ou les pouvoirs publics peuvent faire valoir que certains éléments n'entrent pas dans le champ de cette définition et peuvent donc être transférés ou utilisés sans donner lieu à une rémunération distincte, même si cette utilisation ou ce transfert ferait l'objet d'une rémunération en cas de transaction entre des entreprises indépendantes. À l'inverse, si l'on retient une définition trop large, les contribuables ou les pouvoirs publics peuvent faire valoir que l'utilisation ou le transfert d'un élément dans le cadre de transactions entre entreprises associés doit donner lieu à une rémunération dans des circonstances où cette rémunération n'existerait pas dans le cadre de transactions entre des entreprises indépendantes.

6.6 Dans ces Principes, par conséquent, le terme «incorporel» désigne une chose qui n'est pas un actif corporel ni un actif financier ${ }^{1}$, qui peut être possédée ou contrôlée aux fins d'utilisation dans le cadre d'activités commerciales, et dont l'utilisation ou le transfert serait rémunéré s'il avait lieu dans le cadre d'une transaction entre parties indépendantes dans des circonstances comparables. Dans un cas d'espèce ayant trait à des actifs incorporels, toute analyse des prix de transfert doit être axée non pas sur des définitions comptables ou juridiques, mais sur la détermination des conditions dont seraient convenues des parties indépendantes pour une transaction comparable.

6.7 Les actifs incorporels qu'il importe de prendre en compte aux fins de la détermination des prix de transfert ne sont pas toujours considérés comme des actifs incorporels dans une optique comptable. Ainsi, les coûts associés à la mise au point d'actifs incorporels en interne, prenant par exemple la forme de dépenses de recherche-développement et de publicité, sont parfois passés en charge et non comptabilisés en immobilisations, de sorte que les actifs incorporels résultant de ces dépenses n'apparaissent pas toujours au bilan. Ces actifs incorporels peuvent néanmoins être utilisés pour créer une valeur économique importante, et leur prise en considération peut être nécessaire aux fins de la détermination de prix de transfert. En outre, le surcroît de valeur pouvant découler de la nature complémentaire d'un ensemble d'actifs incorporels exploités ensemble ne transparait pas toujours dans le bilan. En conséquence, pour déterminer si un élément doit ou non être considéré comme un actif incorporel aux fins de l'établissement 
de prix de transfert en vertu de l'article 9 du Modèle de convention fiscale de l'OCDE, on peut s'appuyer sur sa qualification comptable, mais celle-ci ne doit pas être le seul fondement de cette détermination. En outre, la conclusion selon laquelle un élément doit être considéré comme un actif incorporel aux fins de l'établissement de prix de transfert ne détermine pas, ni ne découle de, sa qualification générale d'un point de vue fiscal - en tant que charge ou actif amortissable, par exemple.

6.8 L'existence et la portée d'une protection légale, contractuelle ou autre peut influer sur la valeur d'un élément et sur les revenus qui doivent lui être attribués. L'existence d'une telle protection ne constitue cependant pas une condition nécessaire pour qu'un élément soit qualifié d'actif incorporel aux fins de l'établissement de prix de transfert. De même, si certains actifs incorporels peuvent être identifiés séparément et transférés individuellement, d'autres peuvent n'être transférés qu'avec d'autres actifs industriels ou commerciaux. Par conséquent, il n'est pas nécessaire qu'un élément soit transférable isolément pour qu'il puisse être considéré comme un actif incorporel aux fins de l'établissement de prix de transfert.

6.9 Il est important de distinguer les actifs incorporels des conditions de marché ou de la situation du marché local. Les caractéristiques d'un marché local, telles que le niveau de revenu disponible des ménages sur ce marché ou la taille ou la compétitivité relative de ce marché, ne peuvent être possédées ni contrôlées. Dans certaines circonstances, elles peuvent influer sur la détermination du prix de pleine concurrence pour une transaction donnée et doivent être prises en compte dans le cadre d'une analyse de comparabilité, mais il ne s'agit pas d'actifs incorporels aux fins du chapitre VI. Voir la section D.6 du chapitre I.

6.10 L'identification d'un élément en tant qu'actif incorporel est un processus distinct du processus de détermination du prix correspondant à l'utilisation ou au transfert de cet élément compte tenu des faits et circonstances du cas d'espèce. Suivant le secteur d'activité et les autres faits spécifiques au cas d'espèce, l'exploitation d'actifs incorporels peut représenter une part importante ou modeste de la création de valeur du groupe multinational considéré. Il convient de souligner qu'une rémunération distincte des paiements requis pour les biens ou services n'est pas justifiée en toutes circonstances pour tous les actifs incorporels, et que ces actifs ne débouchent pas tous sur un surcroît de revenus en toutes circonstances. Supposons par exemple qu'une entreprise fournit un service en utilisant un savoir-faire qui n'est pas unique, ce qui implique que d'autres fournisseurs de services comparables disposent d'un savoir-faire comparable. Dans ce cas, même si ce savoir-faire constitue un actif 
incorporel, il peut être estimé que, compte tenu des faits et circonstances, ce savoir-faire ne justifie pas l'attribution d'un surcroît de revenus à l'entreprise, en sus des revenus normaux obtenus par des prestataires indépendants comparables fournissant des services similaires et utilisant un savoir-faire comparable qui n'est pas unique. Voir le paragraphe 1.39. Voir également le paragraphe 6.17 pour une définition des actifs incorporels « uniques ».

6.11 Une grande prudence s'impose pour déterminer si un actif incorporel existe, et s'il a été utilisé ou transféré. Ainsi, les dépenses de recherche-développement n'aboutissent pas toutes à la production ou à l'amélioration d'un actif incorporel, et toutes les activités de commercialisation n'entraînent pas nécessairement la création ou l'amélioration d'un actif incorporel.

6.12 Dans le cadre d'une analyse de prix de transfert ayant trait à des actifs incorporels, il importe d'identifier précisément les actifs incorporels pertinents. L'analyse fonctionnelle doit permettre d'identifier les actifs incorporels pertinents, la façon dont ils contribuent à la création de valeur dans le cadre des transactions examinées, et la façon dont ils interagissent avec d'autres actifs incorporels, des actifs corporels et des activités industrielles ou commerciales pour créer de la valeur. Il peut être justifié d'agréger des actifs incorporels afin de déterminer des conditions de pleine concurrence pour l'utilisation ou le transfert d'actifs incorporels dans certains cas, mais il ne suffit pas d'indiquer que des actifs incorporels vaguement désignés ou indifférenciés ont un effet sur des prix de pleine concurrence ou d'autres conditions. Une analyse fonctionnelle approfondie, y compris une analyse de l'importance que revêtent les actifs incorporels pertinents identifiés dans les activités du groupe multinational à l'échelle mondiale, doit étayer la détermination des conditions de pleine concurrence.

\section{A.2. Application de ce chapitre à d'autres fins fiscales}

6.13 Les instructions figurant dans le présent chapitre sont exclusivement destinées au traitement des questions de prix de transfert. Elles ne sont pas destinées à être appliquées à d'autres fins fiscales. Ainsi, le commentaire de l'article 12 du Modèle de Convention fiscale de l'OCDE contient une analyse précise de la définition du terme «redevances» contenue dans ledit article (paragraphes 8 à 19). La définition des « redevances » qui figure à l'article 12 n'est pas destinée à fournir quelque instruction que ce soit en vue de déterminer si, et si oui à quel prix, l'utilisation ou le transfert d'actifs incorporels doit donner lieu à une rémunération entre parties indépendantes. Elle n'est donc d'aucune utilité pour l'établissement de prix de transfert. En outre, la façon dont une transaction est qualifiée aux fins de la détermination de prix de transfert est 
sans rapport avec la question de savoir si un paiement particulier constitue une redevance ou s'il peut faire l'objet d'une retenue à la source en vertu de l'article 12. Le concept d'actifs incorporels aux fins de l'établissement des prix de transfert et la définition des redevances aux fins de l'article $12 \mathrm{du}$ Modèle de convention fiscale de l'OCDE sont deux notions différentes, qui ne doivent pas nécessairement concorder. Il se peut qu'un paiement entre des entreprises associées soit considéré comme ne constituant pas une redevance aux fins de l'article 12, tout en étant considéré aux fins de l'établissement de prix de transfert comme un paiement auquel peuvent s'appliquer les principes du présent chapitre. On pourrait citer à titre d'exemples certains paiements liés à une survaleur ou à une valeur d'exploitation. Il peut également arriver qu'un paiement légitimement considéré comme une redevance en vertu de l'article 12 d'une convention applicable ne constitue pas une rémunération correspondant à des actifs incorporels aux fins de ce chapitre. On pourrait citer à titre d'exemple des paiements effectués au titre de services techniques. De même, les instructions figurant dans le présent chapitre ne sont pas destinées à être appliquées dans une optique douanière.

6.14 Les instructions figurant dans ce chapitre ne s'appliquent pas non plus à la prise en compte de revenus, la comptabilisation en immobilisation des coûts de mise au point d'actifs incorporels, l'amortissement, ou d'autres questions similaires. Ainsi, un pays peut choisir de ne pas prélever d'impôt sur le transfert de catégories particulières d'actifs incorporels dans certaines circonstances. De même, un pays peut ne pas autoriser l'amortissement du coût de certains éléments acquis qui seraient considérés comme des actifs incorporels suivant les définitions qui figurent dans ce chapitre et dont le transfert peut être assujetti à l'impôt au moment où il a lieu dans le pays du cédant. Il est entendu que des incohérences entre les législations des différents pays concernant ces questions peuvent parfois entraîner une double imposition ou une double exonération.

\section{A.3. Catégories d'actifs incorporels}

6.15 Lors de l'examen des aspects des actifs incorporels touchant aux prix de transfert, il arrive parfois que différentes catégories d'actifs incorporels soient décrites et diverses qualifications appliquées. Des distinctions sont parfois établies entre les actifs incorporels manufacturiers et les actifs incorporels de commercialisation, entre les actifs incorporels " mous» et les actifs incorporels «durs", entre les actifs incorporels courants et spécifiques, ainsi qu'entre d'autres classes et catégories d'actifs incorporels. L'approche présentée dans ce chapitre pour déterminer les prix de pleine concurrence dans les cas se rapportant à des actifs incorporels n'est pas articulée autour de ces catégories. En conséquence, il n'est aucunement tenté dans le cadre de ces Principes de délimiter avec précision différentes 
classes ou catégories d'actifs incorporels, ni de prescrire des résultats axés sur ces catégories.

6.16 Il est cependant couramment fait référence à certaines catégories d'actifs incorporels lors de l'examen des questions de prix de transfert. Pour faciliter ces examens, les définitions de deux termes couramment utilisés, à savoir "actifs incorporels de commercialisation» et "actifs incorporels manufacturiers ", ont été incluses dans le Glossaire et sont mentionnées de temps à autre dans le cadre de ces Principes. Il convient de souligner que les références générales aux actifs incorporels de commercialisation ou manufacturiers ne dispensent pas les contribuables ni les administrations fiscales de leur obligation, dans le cadre d'une analyse des prix de transfert, d'identifier précisément les actifs incorporels pertinents, et que l'utilisation de ces termes n'indique aucunement qu'une approche différente doit être appliquée pour déterminer les conditions du principe de pleine concurrence quant aux transactions portant sur des actifs incorporels de commercialisation ou des actifs incorporels manufacturiers.

\section{Le Glossaire inclus dans ces Principes est modifié, la définition du terme « bien incorporel de commercialisation » étant supprimée et remplacée par celle qui suit :}

« Actif incorporel de commercialisation »

« Actif incorporel (au sens du paragraphe 6.6) lié à des activités de commercialisation, qui contribue à l'exploitation commerciale d'un bien ou d'un service, et/ou a une valeur promotionnelle importante pour le bien ou service concerné. Suivant le contexte, les actifs incorporels de commercialisation peuvent être, par exemple, des marques de fabrique, des noms commerciaux, des listes de clients, des relations avec la clientèle, ainsi que des données exclusives sur des marchés ou des clients qui sont utilisés pour ou contribuent à la commercialisation et la vente de biens ou services à des clients. »

6.17 Dans certains cas, ces Principes font référence à des actifs incorporels « uniques et de valeur». Les actifs incorporels « uniques et de valeur " sont ceux (i) qui ne sont pas comparables aux actifs incorporels utilisés par ou dont disposent les parties à des transactions potentiellement comparables, et (ii) dont l'utilisation par une entreprise dans le cadre de ses activités (telles que la production d'articles manufacturier, la fourniture de services, des activités de commercialisation, de vente ou de gestion) est 
censée déboucher sur des avantages économiques futurs supérieurs à ceux qui auraient été escomptés en l'absence de ces actifs incorporels.

\section{A.4. Illustrations}

6.18 Cette section offre des exemples d'éléments qui sont souvent examinés lors des analyses de prix de transfert ayant trait à des actifs incorporels. Ces exemples sont destinés à clarifier les dispositions de la section A.1, mais cette liste ne saurait se substituer à une analyse approfondie. Ces exemples n'ont pas vocation à être exhaustifs ni à fournir une liste complète des éléments pouvant constituer ou non des actifs incorporels. De nombreux éléments ne figurant pas dans cette liste d'exemples peuvent être des actifs incorporels aux fins de la détermination de prix de transfert. Les exemples figurant dans cette section doivent être adaptés à l'environnement législatif et réglementaire spécifique de chaque pays. En outre, il faut examiner et évaluer les exemples qui figurent dans cette section dans le contexte de l'analyse de comparabilité (notamment fonctionnelle) de la transaction contrôlée en vue de mieux comprendre comment des actifs incorporels spécifiques et des éléments non considérés comme des actifs incorporels contribuent à la création de valeur dans le contexte des activités du groupe multinational à l'échelle mondiale. Il convient de souligner qu'une référence générale à un élément figurant dans cette liste d'exemples ne dispense pas les contribuables ni les administrations fiscales de leur obligation, dans le cadre d'une analyse des prix de transfert, d'identifier précisément les actifs incorporels pertinents sur la base des instructions figurant dans la section A.1.

\section{(i)}

\section{Brevets}

6.19 Un brevet est un instrument juridique qui confère à son détenteur le droit exclusif d'utiliser une invention donnée pendant une durée limitée dans une zone géographique spécifique. Un brevet peut porter sur un objet physique ou un procédé. Les inventions brevetables sont souvent le résultat d'activités risquées et coûteuses de recherche-développement. Dans certaines circonstances, toutefois, des dépenses limitées de recherche-développement peuvent déboucher sur des inventions brevetables de grande valeur. L'auteur d'un brevet peut tenter de récupérer ses coûts de mise au point (et de dégager un revenu) en vendant les produits couverts par le brevet, en concluant avec des tiers des accords de licence leur permettant d'utiliser l'invention brevetée, ou en vendant purement et simplement le brevet considéré. L'exclusivité accordée par un brevet peut, dans certaines circonstances, permettre à son détenteur de tirer un surcroît de revenus de l'utilisation de son invention. Dans d'autres cas, une invention brevetée peut conférer à son détenteur des avantages en termes de coûts dont ne disposent pas ses 
concurrents. Dans d'autres situations encore, il arrive que les brevets ne confèrent aucun avantage commercial important. Les brevets sont des actifs incorporels au sens de la section A.1.

\section{(ii)}

\section{Savoir-faire et secrets industriels ou commerciaux}

6.20 Le savoir-faire et les secrets industriels ou commerciaux sont des informations ou des connaissances exclusives qui facilitent ou améliorent une activité commerciale, mais qui ne sont pas enregistrées en vue de leur protection comme c'est le cas pour un brevet ou une marque de fabrique. Le savoir-faire et les secrets industriels ou commerciaux consistent généralement en des informations non divulguées de nature industrielle, commerciale ou scientifique ayant trait à une expérience acquise, qui trouvent une application pratique dans l'exploitation d'une entreprise. Le savoir-faire et les secrets industriels ou commerciaux peuvent avoir trait à des activités manufacturières, de commercialisation, de recherchedéveloppement, ou à toute autre activité commerciale. La valeur du savoirfaire et des secrets industriels ou commerciaux dépend souvent de la capacité de l'entreprise considérée de préserver leur confidentialité. Dans certains secteurs, la divulgation des informations nécessaires à l'obtention d'une protection par brevet pourraient aider les concurrents à mettre au point d'autres solutions. En conséquence, une entreprise peut, pour des raisons commerciales, choisir de ne pas faire enregistrer un savoir-faire brevetable, qui peut néanmoins contribuer de manière importante à la réussite de l'entreprise. La nature confidentielle du savoir-faire et des secrets industriels ou commerciaux peut être protégée dans une certaine mesure par (i) les dispositions législatives sur la concurrence déloyale ou similaires, (ii) les contrats de travail et (iii) les obstacles économique et technologiques à la concurrence. Le savoir-faire et les secrets industriels ou commerciaux sont des actifs incorporels au sens de la section A.1.

\section{(iii) Marques de fabrique, noms commerciaux et marques} commerciales

6.21 Une marque de fabrique est un nom, un symbole, un logo ou une illustration spécifique que son propriétaire peut utiliser pour distinguer ses biens et services de ceux d'autres entités. Les droits exclusifs attachés aux marques de fabrique sont souvent confirmés par le biais d'un système d'enregistrement. Le titulaire d'une marque de fabrique peut empêcher des tiers de l'utiliser d'une façon qui sèmerait la confusion sur le marché. L'enregistrement d'une marque de fabrique peut demeurer valable indéfiniment si cette marque est constamment utilisée et si cet enregistrement est dûment renouvelé. Une marque de fabrique peut être créée pour des biens ou des services, et peut s'appliquer à un bien ou service 
spécifique ou à une ligne de biens ou services. C'est peut-être sur le marché de la consommation finale que les marques de fabrique sont les plus familières, mais elles peuvent exister à tous les stades de commercialisation. Les marques de fabrique sont des actifs incorporels au sens de la section A.1.

6.22 Un nom commercial (qui est souvent, mais pas toujours, le nom d'une entreprise) peut avoir la même force de pénétration du marché qu'une marque de fabrique et peut, de fait, être enregistré sous une forme spécifique comme une marque de fabrique. Les noms commerciaux de certaines entreprises multinationales peuvent être aisément reconnus, et peuvent être utilisés pour la commercialisation de différents biens et services. Les noms commerciaux sont des actifs incorporels au sens de la section A.1.

6.23 Les termes «marque commerciale », « marque de fabrique » et «nom commercial» sont parfois utilisés indifféremment l'un pour l'autre. Dans d'autres contextes, une marque commerciale désigne une marque de fabrique ou un nom commercial investi d'une importance sociale et commerciale. Une marque commerciale peut, en fait, représenter une combinaison d'actifs incorporels et/ou d'autres éléments, qui peuvent être, entre autres, des marques de fabriques, des noms commerciaux, des relations avec la clientèle, des éléments de notoriété et une survaleur. Il peut être parfois difficile, voire impossible de dissocier ou transférer séparément les différents éléments constitutifs de la valeur d'une marque commerciale. Une marque commerciale peut consister en un seul actif incorporel, ou un ensemble d'actifs incorporels, au sens de la section A.1.

\section{(iv) Droits conférés par les contrats et concessions}

6.24 Les permis et concessions publiques peuvent être importants pour une activité donnée et correspondre à un large éventail de relations commerciales. Ils peuvent recouvrir, entre autres, l'octroi par une administration de droits d'exploitation de ressources naturelles ou de biens publics spécifiques (tel que l'attribution d'une bande de fréquences du spectre radioélectrique), ou du droit d'exercer des activités industrielles ou commerciales spécifiques. Les permis et concessions publiques sont des actifs incorporels au sens de la section A.1. Néanmoins, les permis et concessions publiques doivent être distingués des obligations d'immatriculation des sociétés qui constituent un préalable à l'exercice d'activités dans une juridiction particulière. Ces obligations ne sont pas des actifs incorporels au sens de la section A.1.

6.25 Les droits conférés par les contrats peuvent également être importants pour une activité donnée et correspondre à un large éventail de 
relations commerciales. Ils peuvent recouvrir, entre autres, les contrats conclus avec des fournisseurs et des clients essentiels, ainsi que les accords conclus concernant la fourniture de services par un ou plusieurs employés. Les droits conférés par les contrats sont des actifs incorporels au sens de la section A.1.

\section{(v) Licences et droits limités similaires sur des actifs incorporels}

6.26 Des droits limités sur des actifs incorporels sont couramment transférés au moyen d'une licence ou d'autres dispositions contractuelles similaires, qu'elles soient écrites, orales ou implicites. Ces droits concédés sous licence peuvent être limités en termes de domaine d'utilisation, de conditions d'utilisation, de zone géographique ou sur d'autres plans. Ces droits limités sur des actifs incorporels constituent eux-mêmes des actifs incorporels au sens de la section A.1.

\section{(vi) Survaleur et valeur d'exploitation}

6.27 Suivant le contexte, le terme survaleur peut être utilisé pour faire référence à différents concepts. Dans certains contextes d'évaluation des entreprises et d'évaluation comptable, la survaleur correspond à la différence entre la valeur globale d'une entreprise en exploitation et la somme des valeurs de tous ses actifs corporels et incorporels identifiables séparément. Par ailleurs, la survaleur est parfois décrite comme une représentation des avantages économiques futurs associés à des actifs industriels ou commerciaux qui ne sont pas identifiés individuellement ni comptabilisés séparément. Dans d'autres contextes encore, la survaleur fait référence aux futures transactions escomptées avec les clients existants. Le terme «valeur d'exploitation » est parfois défini comme la valeur de l'ensemble des actifs d'une entreprise en exploitation, qui excède la somme des valeurs de ces différents actifs considérés séparément. Il est généralement admis que la survaleur et la valeur d'exploitation ne peuvent être dissociés ou transférés séparément des autres actifs industriels ou commerciaux. Voir les paragraphes 9.93 à 9.95 pour un examen de la question connexe du transfert de l'ensemble des éléments d'une activité dans le contexte d'une réorganisation d'entreprise.

6.28 Aux fins de ce chapitre, il n'est pas nécessaire d'élaborer une définition précise de la survaleur ni de la valeur d'exploitation dans l'optique de la détermination des prix de transfert, ni d'établir dans quelles circonstances une survaleur ou une valeur d'exploitation peut constituer ou non un actif incorporel. Il importe cependant de reconnaître qu'une part importante et monétairement significative de la rémunération versée entre 
des entreprises indépendantes lorsque certains ou tous les actifs d'une entreprise en exploitation sont transférés peut représenter une rémunération correspondant à un élément évoqué dans l'une ou l'autre des différentes descriptions de la survaleur ou de la valeur d'exploitation. Lorsque des transactions similaires se déroulent entre des entreprises associées, cette valeur doit être prise en compte dans le cadre de la détermination d'un prix de pleine de concurrence pour la transaction considérée. Lorsque la valeur de notoriété parfois désignée par le terme survaleur est transférée à une entreprise associée, ou partagée avec elle, dans le contexte du transfert ou de la concession sous licence d'une marque de fabrique ou d'autres actifs incorporels, il faut prendre en compte cette valeur de notoriété pour déterminer le niveau adéquat de la rémunération correspondante. Si les caractéristiques d'une entreprise, telles que la réputation de produire des biens ou de fournir des services de haute qualité, lui permettent de facturer des prix plus élevés qu'une entité n'ayant pas une telle réputation, et si ces caractéristiques peuvent être considérées comme constitutives d'une survaleur ou d'une valeur d'exploitation suivant l'une ou l'autre des définitions de ces termes, il faut prendre en compte ces caractéristiques pour déterminer les prix de pleine concurrence correspondant à la vente de biens ou à la fourniture de services entre des entreprises associées, qu'elles soient ou non considérées comme des éléments de survaleur. En d'autres termes, il ne suffit pas qu'une contribution de valeur d'une partie à une autre soit qualifiée d'élément constitutif d'une survaleur ou d'une valeur d'exploitation pour qu'il n'y ait pas lieu de rémunérer cette contribution. Voir le paragraphe 6.2 .

6.29 Le fait que la survaleur et la valeur d'acquisition doivent être prises en compte pour la détermination du prix des transactions ne signifie aucunement que les mesures résiduelles de la survaleur calculées à des fins spécifiques d'évaluation comptable ou d'évaluation d'entreprise sont nécessairement des mesures appropriées du prix qui serait payé pour les activités ou droits concédés sous licence, ainsi que la survaleur ou valeur d'exploitation qui leur est associée, par des parties indépendantes. Les mesures de la survaleur et de la valeur d'exploitation établies dans le cadre d'une évaluation comptable ou d'une évaluation d'entreprise ne correspondent pas, en règle générale, au prix de pleine concurrence d'une survaleur ou d'une valeur d'exploitation transférée dans le cadre d'une analyse de prix de transfert. Suivant les faits et les circonstances, néanmoins, les évaluations comptables et les informations sur lesquelles elles reposent peuvent fournir un point de départ utile pour réaliser une analyse de prix de transfert. Compte tenu de l'absence d'une définition unique et précise de la survaleur, il est essentiel que les contribuables et les administrations fiscales décrivent les actifs incorporels spécifiquement concernés par une analyse 
des prix de transfert, et déterminent si ces actifs incorporels donneraient lieu à une rémunération entre des entreprises indépendantes dans des circonstances comparables.

\section{(vii) Synergies de groupe}

6.30 Dans certaines circonstances, les synergies de groupe contribuent au niveau des revenus engrangés par un groupe multinational. Ces synergies de groupe peuvent prendre de nombreuses formes différentes, telles qu'une gestion optimisée, une élimination des doubles emplois coûteux, des systèmes intégrés, une puissance d'achat, une capacité d'emprunt, etc. Ces caractéristiques peuvent influer sur la détermination des conditions de pleine concurrence pour des transactions contrôlées, et doivent être prises en compte en tant que facteurs de comparabilité aux fins de l'établissement de prix de transfert. Dans la mesure où ils ne sont ni possédés ni contrôlés par une entreprise, il ne s'agit pas d'actifs incorporels au sens de la section A.1. Voir les paragraphes 1.98 à 1.114 pour un examen du traitement réservé aux synergies de groupe dans l'optique de la détermination de prix de transfert.

\section{(viii) Caractéristiques spécifiques des marchés}

6.31 Les caractéristiques spécifiques d'un marché donné peuvent influer sur les conditions du principe de pleine concurrence des transactions sur ce marché. Ainsi, le fort pouvoir d'achat des ménages sur un marché donné peut avoir une incidence sur les prix facturés pour certains biens de consommation de luxe. De même, des coûts de main-d'œuvre faibles, la proximité de certains marchés, des conditions météorologiques favorables et d'autres facteurs du même ordre peuvent influer sur les prix pratiqués pour des biens et services spécifiques sur un marché donné. Ces caractéristiques spécifiques d'un marché ne peuvent cependant être possédées ni contrôlées, et ne constituent donc pas des actifs incorporels au sens de la section A.1, et elles doivent être prises en compte dans le cadre d'une analyse de prix de transfert par le biais de l'analyse de comparabilité requise. Voir les paragraphes 1.80 à 1.92 et les paragraphes 9.148 à 9.153 sur les instructions concernant le traitement des caractéristiques spécifiques des marchés dans l'optique de la détermination de prix de transfert.

\section{[B. Propriété des actifs incorporels et transactions portant sur la mise au point, l'amélioration, l'entretien, la protection et l'exploitation d'actifs incorporels}

6.32 Dans les dossiers de prix de transfert portant sur des actifs incorporels, la détermination de l'entité (ou des entités) d'un groupe multinational qui est in fine en droit de bénéficier des revenus tirés par le 
groupe de l'exploitation des actifs incorporels considérés est cruciale ${ }^{2}$. Une question connexe est de savoir quelle entité (ou quelles entités) du groupe doit in fine assumer les coûts, les investissements et autres charges associés à la mise au point, à l'amélioration, à l'entretien, à la protection et à l'exploitation des actifs incorporels considérés. Bien que le propriétaire légal d'un actif incorporel puisse recevoir les revenus provenant de l'exploitation de cet actif, d'autres membres du groupe multinational dont fait partie ce propriétaire légal peuvent avoir exercé des fonctions, utilisé des actifs ${ }^{3}$ ou assumé des risques qui devraient contribuer à la valeur de l'actif incorporel en question. Les membres du groupe multinational qui exercent ces fonctions, utilisent ces actifs et assument ces risques doivent voir leur contribution rémunérée en vertu du principe de pleine concurrence. La présente section $\mathrm{B}$ confirme qu'in fine, la répartition des revenus tirés par le groupe multinational considéré de l'exploitation d'actifs incorporels, de même que la répartition des coûts et autres charges liés à ces actifs incorporels entre les membres de ce groupe multinational, repose sur la rémunération des membres dudit groupe qui exercent des fonctions, utilisent des actifs ou assument des risques dans le cadre de la mise au point, de l'amélioration, de l'entretien, de la protection et de l'exploitation de ces actifs incorporels suivant les principes décrits aux chapitres I à III.

6.33 Il peut être extrêmement difficile d'appliquer les dispositions des chapitre I à III pour traiter ces questions, pour différentes raisons. Suivant les faits du cas d'espèce portant sur des actifs incorporels, les facteurs suivants, entre autres, peuvent soulever des problèmes :

(i) un manque de comparabilité entre les transactions portant sur des actifs incorporels effectuées entre des entreprises associées et les transactions de ce type pouvant être identifiées entre des entreprises indépendantes ;

(ii) un manque de comparabilité entre les actifs incorporels en question ;

(iii) la possession et/ou l'utilisation de différents actifs incorporels par différentes entreprises associées appartenant au groupe multinational considéré ;

(iv) la difficulté d'isoler l'effet induit par tel ou tel actif incorporel sur les revenus du groupe multinational considéré ;

(v) le fait que différents membres d'un groupe multinational puissent exercer des activités liées à la mise au point, l'amélioration, 
l'entretien, la protection et l'exploitation d'un actif incorporel, souvent d'une façon et avec un niveau d'intégration que l'on n'observe pas entre des entreprises indépendantes ;

(vi) les fait que les contributions de différents membres du groupe multinational considéré à la création de valeur incorporelle puissent avoir lieu au cours d'années différentes de celles pendant lesquelles sont réalisés les éventuels gains associés ; et

(vii) le fait que les structures du contribuable puissent être fondées sur des dispositions contractuelles conclues entre des entreprises associées qui dissocient la propriété, la prise en charge du risque et/ou le financement des investissements en actifs incorporels de l'exercice de fonctions importantes, du contrôle des risques et des décisions liées aux investissements d'une façon que l'on n'observe pas dans le cadre de transactions entre des entreprises indépendante, et qui peut contribuer à des phénomènes d'érosion de la base d'imposition et de transfert de bénéfices.

Nonobstant ces problèmes potentiels, l'application du principe de pleine concurrence et des dispositions des chapitres I à III dans un cadre établi peut, dans de nombreux cas, permettre une attribution appropriée des revenus tirés par le groupe multinational considéré de l'exploitation d'actifs incorporels.

6.34 Le cadre d'analyse des transactions portant sur des actifs incorporels repose sur les étapes suivantes :

(i) identifier le propriétaire légal des actifs incorporels considérés sur la base des conditions des accords juridiques pertinents, notamment des immatriculations, des accords de licence et autres contrats pertinents, ainsi que d'autres indices de propriété légale ;

(ii) identifier les parties qui exercent des fonctions (en particulier les fonctions importantes décrites au paragraphe 6.56), utilisent des actifs et assument des risques liés à la mise au point, à l'amélioration, à l'entretien, à la protection et à l'exploitation des actifs incorporels considérés, au moyen d'une analyse fonctionnelle ;

(iii) confirmer l'existence d'une cohérence entre le comportement des parties et les conditions des accords juridiques pertinents en ce qui 
concerne la propriété des actifs incorporels considérés, au moyen d'une analyse fonctionnelle approfondie ;

(iv) identifier les transactions contrôlées liées à la mise au point, à l'amélioration, à l'entretien, à la protection et à l'exploitation des actifs incorporels considérés compte tenu de la propriété légale de ces actifs établie par les immatriculations et contrats pertinents, ainsi que du comportement des parties, notamment de leurs contributions respectives en termes de fonctions exercées, d'actifs utilisés, de risques assumés et d'autres facteurs contribuant à la création de valeur;

(v) si possible, déterminer les prix de pleine concurrence de ces transactions sur la base des contributions de chaque partie en termes de fonctions exercées, d'actifs utilisés et de risques assumés ; et

(vi) dans les circonstances décrites aux paragraphes 1.64 à 1.69 , requalifier les transactions si nécessaire pour refléter les conditions du principe de pleine concurrence.

\section{B.1. Propriété des actifs incorporels et dispositions contractuelles relatives aux actifs incorporels}

6.35 Les droits reconnus par la loi et les dispositions contractuelles constituent le point de départ de toute analyse de prix de transfert relative à des transactions portant sur des actifs incorporels. Les conditions d'une transaction peuvent être déduites de contrats écrits, de documents publics tels que les enregistrements de brevets ou de marques de fabrique, ou de la correspondance et/ou d'autres communications entre les parties considérées. Les contrats peuvent décrire les rôles, responsabilités et droits des entreprises associés en matière d'actifs incorporels. Ils peuvent indiquer quelle entité (ou quelles entités) apporte des fonds, réalise des activités de recherche-développement, entretient et protège les actifs incorporels, et exerce les fonctions nécessaires à l'exploitation des actifs incorporels, telles que la fabrication, la commercialisation et la distribution. Ils peuvent indiquer comment les recettes et les dépenses du groupe multinational associées aux actifs incorporels doivent être réparties, et peuvent spécifier la forme et le montant des paiements devant être reçus par tous les membres du groupe pour leurs contributions. Les prix et autres conditions précisés dans ces contrats peuvent être conformes ou non au principe de pleine concurrence. 
6.36 Lorsqu'il n'existe pas de dispositions écrites, lorsque les dispositions contractuelles sont ambiguës ou incomplètes, ou lorsque la nature factuelle de la transaction telle qu'elle ressort du comportement des parties n'est pas conforme aux contrats écrits, il convient de déduire les conditions d'une transaction du comportement des parties ainsi que des principes économiques qui régissent habituellement les relations entre des entreprises indépendantes. Il est donc souhaitable que les entreprises associées conservent une trace écrite de leurs décisions et de leurs intentions concernant l'attribution des droits importants sur des actifs incorporels. Ces décisions et intentions, notamment les accords écrits, doivent généralement être en place lorsque ou avant que des entreprises associées procèdent à des transactions débouchant sur la mise au point, l'amélioration, l'entretien, la protection ou l'exploitation d'actifs incorporels.

6.37 Le droit d'utiliser certains types d'actifs incorporels peut être protégé par des systèmes d'enregistrement et des lois spécifiques concernant la propriété intellectuelle. Les brevets, les marques de fabrique et les droits d'auteur sont des exemples d'actifs incorporels de ce type. En règle générale, le titulaire légal de ces actifs incorporels dispose du droit exclusif sur le plan juridique et commercial d'utiliser ces actifs, ainsi que du droit d'empêcher des tiers de les utiliser ou de porter quelque atteinte que ce soit aux droits sur ces actifs. Ces droits du titulaire légal peuvent être conférés pour une zone géographique spécifique et/ou pour une période spécifique.

6.38 Il existe également des actifs incorporels qui ne peuvent être protégés dans le cadre de systèmes particulier d'enregistrement de la propriété intellectuelle, mais qui sont protégés contre toute imitation ou appropriation non autorisée par les règles de droit sur la concurrence déloyale ou d'autres dispositions juridiques applicables, ou par contrat. La présentation commerciale, les secrets industriels ou commerciaux et le savoir-faire peuvent entrer dans cette catégorie d'actifs incorporels.

6.39 La portée et la nature de la protection offerte par le droit applicable peut varier d'un pays à l'autre, de même que les conditions suivant lesquelles cette protection est assurée. Ces divergences peuvent être dues soit à des différences de fond entre pays concernant le droit relatif à la propriété intellectuelle, soit à des différences pratiques concernant la mise en application locale de ces lois. Ainsi, le bénéfice d'une protection juridique peut être subordonné pour certains actifs incorporels à certaines conditions, telles qu'une utilisation commerciale persistante de l'actif incorporel considéré ou le renouvellement en temps voulu de son enregistrement. Cela signifie que dans certaines circonstances ou juridictions, la protection dont 
fait l'objet un actif incorporel peut être extrêmement limitée en droit ou en pratique.

6.40 Le propriétaire légal, déterminé suivant les principes énoncés aux paragraphes 1.52 et 1.53 , sera considéré comme le propriétaire de l'actif incorporel considéré aux fins de l'établissement des prix de transfert. Si aucun propriétaire légal n'est identifié en vertu du droit ou des contrats applicables, le membre du groupe multinational qui, compte tenu des faits et circonstances, contrôle les décisions concernant l'exploitation de l'actif incorporel considéré et peut en pratique restreindre son utilisation par des tiers sera considéré comme le propriétaire légal de cet actif aux fins de la détermination des prix de transfert.

6.41 Dans l'optique de l'identification du propriétaire légal d'actifs incorporels, un actif incorporel et toute licence relative à celui-ci sont considérés comme différents aux fins de la détermination des prix de transfert, chacun ayant un propriétaire différent. Voir le paragraphe 6.26. Ainsi, la société A, qui le propriétaire légal d'une marque de fabrique, peut octroyer une licence exclusive à la société $\mathrm{B}$ pour la fabrication, la commercialisation et la vente de biens faisant appel à cette marque de fabrique. Un actif incorporel, la marque de fabrique, est la propriété légale de la société A. Un autre actif incorporel, la licence d'utilisation de la marque de fabrique en relation avec la fabrication, la commercialisation et la distribution de produits de marque, est la propriété légale de la société B. Suivant les faits et circonstances, les activités de commercialisation entreprises par la société B en vertu de sa licence peuvent avoir une incidence sur la valeur de l'actif incorporel sous-jacent dont la société A est légalement propriétaire, la valeur de la licence de la société $\mathrm{B}$, ou les deux.

6.42 La détermination de la propriété légale des actifs considérés constitue une première étape importante de l'analyse, mais cette détermination est un processus distinct de la question de la rémunération suivant le principe de pleine concurrence. Aux fins de la détermination des prix de transfert, la propriété légale d'un actif incorporel ne confère, en soi, aucun droit de conserver in fine les revenus tirés par le groupe multinational considéré de l'exploitation de cet actif, même si ces revenus peuvent initialement être perçus par son propriétaire légal en raison de son droit légal ou contractuel d'exploiter l'actif incorporel en question. Les revenus qui sont in fine conservés par le propriétaire légal ou qui lui sont attribués dépendent des fonctions qu'il exerce, des actifs qu'il utilise et des risques qu'il assume, ainsi que des contributions apportées par les autres membres du groupe multinational considéré compte tenu des fonctions qu'ils exercent, des actifs qu'ils utilisent et des risques qu'ils assument. Ainsi, dans le cas d'un actif 
incorporel mis au point en interne, si le propriétaire légal n'exerce aucune fonction, n'utilise aucun actif et n'assume aucun risque pertinents, et s'il intervient uniquement en tant qu'entité détentrice de titre, le propriétaire légal n'aura droit in fine à aucune part des revenus tirés par le groupe multinational de l'exploitation de l'actif incorporel considéré, en dehors de l'éventuelle rémunération de pleine concurrence correspondant à la détention du titre.

6.43 La propriété légale et les relations contractuelles servent simplement de points de référence pour identifier et analyser les transactions contrôlées portant sur l'actif incorporel considéré, et pour déterminer la rémunération appropriée des membres du groupe contrôlé concernant ces transactions. La détermination de la propriété légale, conjuguée à l'identification et à la rémunération des fonctions exercées, des actifs utilisés et des risques assumés par l'ensemble des membres contributeurs qui sont pertinents, offre le cadre d'analyse qui permet de déterminer les prix de pleine concurrence et les autres conditions des transactions portant sur des actifs incorporels. Comme pour tout autre type de transaction, l'analyse doit prendre en compte l'ensemble des faits et circonstances pertinents dans le cas d'espèce, et la détermination des prix doit refléter les autres solutions réalistes envisageables par les membres concernés du groupe. Les principes énoncés dans ce paragraphe sont illustrés par les exemples 1 à 7 qui figurent dans l'annexe au chapitre VI.

6.44 Dans la mesure où les membres d'un groupe multinational n'ont aucune certitude au moment où ils prennent des décisions concernant des actifs incorporels sur leurs résultats effectifs ni sur les conséquences éventuelles qu'auront au fil du temps les risques associés à la mise au point ou à l'acquisition de ces actifs incorporels, il importe de distinguer (a) la rémunération anticipée (ou ex ante), qui désigne les revenus futurs sur lesquels table un membre du groupe multinational au moment d'une transaction; et (b) la rémunération effective (ou ex post), qui désigne les revenus effectivement perçus par un membre du groupe multinational grâce à l'exploitation de l'actif incorporel considéré.

6.45 La rémunération devant être versée aux membres du groupe multinational considéré qui contribuent à la mise au point, à l'amélioration, à l'entretien, à la protection et à l'exploitation des actifs incorporels est généralement déterminée sur une base ex ante. Autrement dit, elle est établie au moment où la transaction est conclue et avant que les conséquences éventuelles des risques associés à l'actif incorporel considéré ne se matérialisent. Cette rémunération peut être fixe ou conditionnelle. 
L'attribution des bénéfices (ou pertes) effectifs ex post dépendra des faits et circonstances du cas d'espèce.

6.46 Une question importante est de savoir comment déterminer la rémunération de pleine concurrence qui revient aux membres d'un groupe en contrepartie des fonctions qu'ils exercent, des actifs qu'ils utilisent et des risques qu'ils assument dans le cadre constitué par les arrangements contractuels du contribuable, la propriété légale des actifs incorporels et le comportement des parties. La section B.2. porte sur l'application du principe de pleine concurrence aux situations relatives à des actifs incorporels. Sauf indication contraire, lorsqu'il est fait mention de revenus de pleine concurrence ou d'une rémunération de pleine concurrence dans la section B.2., il s'agit de revenus anticipés (ex ante) dans le premier cas et d'une rémunération anticipée (ex ante) dans le second cas.

\section{B.2. $\quad$ Fonctions, actifs et risques liés à des actifs incorporels}

6.47 Comme indiqué plus haut, déterminer qu'un membre particulier d'un groupe multinational est le propriétaire légal d'actifs incorporels n'implique pas, en soi, que ce membre est en droit in fine de conserver ou de se voir attribuer les recettes qu'il peut engranger au départ du fait de son droit commercial d'exploitation de ces actifs incorporels, et cela ne signifie pas non plus nécessairement que ce propriétaire légal peut prétendre à un revenu après avoir rémunéré les autres membres du groupe multinational considéré pour les contributions qu'ils ont apportées en exerçant des fonctions, en utilisant des actifs et en assumant des risques. Autrement dit, après avoir rémunéré de manière appropriée les autres membres du groupe multinational au regard des fonctions qu'ils ont exercées, des actifs qu'ils ont utilisés et des risques qu'ils ont assumés, le propriétaire légal de l'actif incorporel considéré peut avoir des revenus liés audit actif positifs, négatifs ou nuls suivant les faits du cas d'espèce.

6.48 Lors de la détermination des prix de pleine concurrence concernant des transactions entre entreprises associées, les contributions des membres du groupe multinational liées à la création de valeur incorporelle doivent être prises en considération et faire l'objet d'une rémunération appropriée. En vertu du principe de pleine concurrence et des prescriptions énoncées aux chapitres I à III, tous les membres du groupe multinational considéré doivent recevoir une rémunération appropriée s'ils exercent des fonctions, utilisent des actifs ou assument des risques en relation avec la mise au point, l'amélioration, l'entretien, la protection ou l'exploitation d'actifs incorporels. Il est donc nécessaire de déterminer, au moyen d'une analyse fonctionnelle, quel membre (ou membres) exerce des fonctions de mise au point, d'amélioration, d'entretien, de protection et d'exploitation des 
actifs incorporels ou exerce un contrôle sur ces fonctions, quel membre (ou membres) apporte les financements et autres actifs nécessaires, et quel membre (ou membres) contrôle et assume les différents risques associés à l'actif incorporel considéré. Naturellement, dans chacun de ces domaines, il peut s'agir ou non du propriétaire légal de l'actif incorporel considéré. Comme indiqué au paragraphe 6.130, il importe également lors de la détermination de la rémunération de pleine concurrence des fonctions exercées, des actifs utilisés et des risques assumés de prendre en considération les facteurs de comparabilité pouvant contribuer à la création de valeur ou à la production de revenus tirés par le groupe multinational de l'exploitation des actifs incorporels considérés, pour déterminer les prix des transactions concernées.

6.49 L'importance relative de ces contributions à la création de valeur incorporelle par les membres du groupe sous la forme de fonctions exercées, d'actifs utilisés ou de risques assumés variera en fonction des circonstances. Supposons, par exemple, qu'un actif incorporel dont la mise au point est achevée et qui est actuellement exploitable soit acheté à une tierce partie par un membre du groupe multinational, et exploité dans le cadre de fonctions de fabrication et de distribution exercées par d'autres membres de ce groupe, tout en étant activement géré et contrôlé par l'entité acquéreuse de cet actif incorporel. On suppose que cet actif incorporel ne nécessite aucune activité de mise au point, exige peu ou pas d'activités d'entretien ou de protection, et a une utilité sans doute limitée en dehors du domaine d'exploitation ciblé au moment de l'acquisition. Il n'y pas de risque de mise au point associé à cet actif incorporel, même si son exploitation peut comporter d'autres risques. Les fonctions essentielles exercées par l'acquéreur sont celles nécessaires pour sélectionner l'actif incorporel le plus approprié sur le marché, pour analyser ses avantages potentiels en cas d'utilisation par le groupe multinational considéré, et pour décider de l'acheter ou non. L'actif essentiel utilisé réside dans les fonds nécessaires à l'achat de cet actif incorporel. Si l'acquéreur est capable d'exercer et exerce effectivement toutes les fonctions essentielles décrites, on peut raisonnablement en conclure que, après avoir procédé à une rémunération de pleine concurrence des fonctions de fabrication et de distribution exercées par d'autres entreprises associées, le propriétaire serait en droit de conserver ou de se voir attribuer le bénéfice ou la perte éventuelle résultant de l'exploitation de l'actif incorporel à la suite de son acquisition. L'application des chapitres I à III peut être relativement évidente dans un scénario aussi simple, mais l'analyse peut être plus difficile dans des situations où :

(i) des actifs incorporels sont mis au point en interne par un groupe multinational, en particulier lorsque ces actifs incorporels sont 
transférés entre des entreprises associées alors qu'ils sont encore en cours de développement ;

(ii) des actifs incorporels achetés ou mis au point en interne servent de plateforme pour d'autres activités de développement ; ou

(iii) d'autres aspects, tels que la commercialisation ou la fabrication, sont particulièrement importants pour la création de valeur.

Les instructions d'application générale qui figurent ci-après sont particulièrement utiles pour, et essentiellement axées sur, ces cas d'espèce plus délicats.

\section{Exercice et contrôle des fonctions}

6.50 Suivant les principes énoncés aux chapitres I à III, chaque membre d'un groupe multinational doit recevoir une rémunération de pleine concurrence pour les fonctions qu'il exerce. Dans les cas d'espèce portant sur des actifs incorporels, cela recouvre les fonctions liées à la mise au point, à l'amélioration, à l'entretien, à la protection et à l'exploitation d'actifs incorporels. L'identité du membre (ou des membres) du groupe exerçant des fonctions liées à la mise au point, à l'amélioration, à l'entretien, à la protection et à l'exploitation de ces actifs incorporels. L'identité du membre (ou des membres) du groupe multinational considéré exerçant des fonctions liées à la mise au point, à l'amélioration, à l'entretien, à la protection et à l'exploitation d'actifs incorporels est donc un élément essentiel à prendre en compte pour déterminer les prix des transactions contrôlées, et pour établir quelle entité (ou entités) sera in fine en droit de bénéficier des revenus tirés par le groupe de l'exploitation des actifs incorporels en question.

6.51 La nécessité de veiller à ce que tous les membres du groupe multinational considéré soient rémunérés de manière appropriée pour les fonctions qu'ils exercent implique que pour être en droit de conserver in fine tous les revenus tirés de l'exploitation d'actifs incorporels, le propriétaire légal de ces actifs doit exercer toutes les fonctions, apporter tous les actifs utilisés et assumer tous les risques liés à la mise au point, à l'amélioration, à l'entretien, à la protection et à l'exploitation des actifs incorporels en question. Cela ne signifie pas cependant que les entreprises associées constituant un groupe multinational doivent structurer leurs activités concernant la mise au point, l'amélioration, l'entretien, la protection ou l'exploitation d'actifs incorporels d'une façon particulière. Il n'est pas essentiel que le propriétaire légal exerce physiquement toutes les fonctions liées à la mise au point, à l'amélioration, à l'entretien, à la protection et à l'exploitation d'un actif incorporel par le biais de ses propres employés pour 
être en droit in fine de conserver ou de se voir attribuer une part des revenus tirés par le groupe multinational considéré de l'exploitation de cet actif incorporel. Dans le cadre de transactions entre des entreprises indépendantes, certaines fonctions sont parfois externalisées à d'autres entités. Un membre d'un groupe multinational qui est le propriétaire légal d'actifs incorporels peut externaliser de manière similaire des fonctions liées à la mise au point, à l'amélioration, à l'entretien, à la protection et à l'exploitation d'actifs incorporels à des entreprises indépendantes ou associées.

6.52 Lorsque des entreprises associées autres que le propriétaire légal d'un actif incorporel exercent des fonctions pertinentes dont on anticipe qu'elles contribueront à la valeur de cet actif, elles doivent obtenir une rémunération de pleine concurrence pour les fonctions qu'elles exercent suivant les principes énoncés dans les chapitres I à III. Pour établir la rémunération de pleine concurrence correspondant à ces contributions fonctionnelles, il faut déterminer s'il existe des transactions comparables sur le marché libre, quelle est l'importance des fonctions exercées pour la création de valeur incorporelle, et quelles sont les options réalistes à la disposition des parties. Les considérations spécifiques décrites aux paragraphes 6.53 à 6.58 doivent également être prises en compte.

6.53 En cas de transaction d'externalisation entre des entreprises indépendantes, on constate généralement qu'une entité exerçant des fonctions au nom du propriétaire légal d'un actif incorporel qui ont trait au développement, à l'amélioration, à l'entretien, à la protection ou à l'exploitation de cet actif opère sous la direction ou le contrôle de ce propriétaire légal. Compte tenu de la nature des relations existant entre des entreprises associées qui sont membres d'un groupe multinational, il peut néanmoins arriver que des fonctions externalisées exercées par des entreprises associées soient contrôlées par une entité autre que le propriétaire légal de l'actif incorporel considéré. Dans ce cas, le propriétaire légal de cet actif incorporel doit également verser une rémunération de pleine concurrence à l'entité qui exerce les fonctions de contrôle liées à la mise au point, à l'amélioration, à l'entretien, à la protection et à l'exploitation de l'actif en question. Pour déterminer quel est le membre du groupe multinational qui contrôle effectivement l'exercice des fonctions considérées, il convient d'appliquer des principes analogues à ceux énoncés aux paragraphes 9.22 à 9.28. L'évaluation de la capacité d'une entité donnée d'exercer un contrôle et des fonctions de contrôle constituera un élément important de l'analyse. 
6.54 Si le propriétaire légal ne contrôle pas et n'exerce pas les fonctions liées à la mise au point, à l'amélioration, à l'entretien, à la protection et à l'exploitation de l'actif incorporel considéré, il n'aura droit à aucun avantage courant attribuable aux fonctions externalisées. Sauf si des transactions ou structures sont non prises en compte ou requalifiées de manière appropriée en vertu des paragraphes 1.64 à 1.69, un propriétaire légal n'exerçant aucune fonction pertinente peut néanmoins avoir droit à une part des revenus tirés par le groupe multinational considéré de l'exploitation des actifs incorporels concernés, à titre de rémunération des actifs qu'il utilise ou des risques qu'il assume, le cas échéant. Voir les paragraphes 6.59 à 6.65. Il n'aura cependant droit à aucune part des revenus liés à l'exercice ou au contrôle des fonctions considérées. En fonction des faits du cas d'espèce, la rémunération de pleine concurrence devant être versée par le propriétaire légal aux autres entreprises associées qui exécutent ou contrôlent des fonctions liées à la mise au point, à l'amélioration, à l'entretien, à la protection ou à l'exploitation des actifs incorporels considérés peut correspondre à n'importe quelle proportion des revenus totaux tirés de l'exploitation de ces actifs incorporels.

6.55 La valeur relative des contributions à la mise au point, à l'amélioration, à l'entretien, à la protection et à l'exploitation d'actifs incorporels varie en fonction des faits du cas d'espèce. Le membre (ou les membres) du groupe multinational considéré dont la contribution est la plus importante dans un cas donné doit recevoir une rémunération d'un niveau plus élevé. Ainsi, si une entreprise se contente de financer des activités de recherche-développement, ses revenus anticipés doivent être plus faibles que si elle finançait ces activités tout en les contrôlant. Toutes choses égales par ailleurs, ces revenus anticipés doivent être encore plus élevés si l'entité concernée finance, contrôle et exerce physiquement les activités de recherche-développement en question.

6.56 Lors de la détermination de la rémunération de pleine concurrence des contributions fonctionnelles des divers membres du groupe multinational considéré, certaines fonctions importantes auront un statut particulier. La nature de ces fonctions importantes dépendra des faits et circonstances de chaque cas d'espèce. Pour les actifs incorporels mis au point en interne, ou pour les actifs incorporels mis au point en interne ou achetés qui servent de plateforme pour d'autres activités de mise au point, ces fonctions particulièrement importantes peuvent être, entre autres, la conception et le contrôle des programmes de recherche et de commercialisation, la fixation des orientations et des priorités en matière de projets créatifs, notamment de l'axe des activités de recherche fondamentale, le contrôle des décisions stratégiques concernant les programmes de mise au 
point d'actifs incorporels, ainsi que la gestion et le contrôle des budgets. Pour tout actif incorporel (qu'il soit mis au point en interne ou acheté), les autres fonctions importantes peuvent également correspondre à la prise de décisions clés concernant la défense et la protection des actifs incorporels, ainsi que le contrôle de qualité courant relatif aux fonctions exercées par des entreprises indépendantes ou associées pouvant avoir un effet sensible sur la valeur de l'actif incorporel considéré. Ces fonctions importantes contribuent généralement de manière significative à la création de valeur incorporelle et, si ces fonctions importantes sont externalisées par le propriétaire légal dans le cadre de transactions entre entreprises associées, l'exercice de ces fonctions doit être rémunéré au moyen d'une part appropriée des revenus tirés par le groupe multinational considéré de l'exploitation des actifs incorporels.

6.57 Dans la mesure où il peut être difficile de trouver des transactions comparables portant sur l'externalisation de ces fonctions importantes, il peut être nécessaire d'utiliser des méthodes de détermination des prix de transfert qui ne sont pas directement fondées sur des comparables, notamment des méthodes de partage des bénéfices et des techniques d'évaluation, afin de rémunérer de manière appropriée l'exercice de ces fonctions importantes. Lorsque le propriétaire légal externalise l'essentiel ou la totalité de ces fonctions importantes à d'autres membres du groupe multinational considéré, il est très incertain que ce propriétaire légal puisse prétendre à une part importante des revenus tirés de l'exploitation des actifs incorporels concernés, après avoir rémunéré les autres membres du groupe pour leurs fonctions. Dans certaines circonstances, il peut également être établi que des entreprises indépendantes n'auraient pas externalisé ces fonctions importantes dans une optique commerciale rationnelle, et que la structure effective adoptée fait obstacle à la détermination d'un prix de transfert approprié, de sorte qu'il est nécessaire de pas tenir compte de cette structure adoptée conformément aux principes énoncés au paragraphe 1.65. Les exemples 17 et 18 qui figurent dans l'annexe au chapitre VI illustrent les principes énoncés dans ce paragraphe.

6.58 Dans la mesure où les fonctions importantes décrites au paragraphe 6.56 contribuent souvent à la gestion des fonctions exercées, actifs utilisés et risques assumés qui sont essentiels pour la réussite de la mise au point, de l'amélioration, de l'entretien, de la protection ou de l'exploitation d'actifs incorporels, et sont par conséquent cruciales pour la création de valeur incorporelle, il est nécessaire d'évaluer avec soin les transactions entre les parties qui exercent ces fonctions importantes et les autres entreprises associées. Une méthode unilatérale de détermination des prix de transfert sera nettement moins fiable si la partie (ou les parties) 
exerçant une part significative de ces fonctions importantes est la partie testée. Voir l'exemple 7.

\section{Utilisation d'actifs}

6.59 Les membres du groupe multinational qui utilisent des actifs dans le cadre du développement, de l'amélioration, de l'entretien, de la protection ou de l'exploitation d'un actif incorporel doivent recevoir en conséquence une rémunération appropriée. Il peut s'agir, sans que cette liste soit limitative, d'actifs incorporels utilisés dans le cadre d'activités de recherche, de mise au point ou de commercialisation (tels que du savoir-faire, des relations avec la clientèle, etc.), d'actifs corporels ou de financements. Un membre d'un groupe multinational peut financer tout ou partie des fonctions de mise au point, d'amélioration, d'entretien et de protection d'un actif incorporel, tandis qu'un ou plusieurs autres membres exercent toutes les fonctions considérées. Lors de l'évaluation du rendement anticipé approprié de ce financement dans de telles circonstances, il convient de prendre en compte le fait que, dans le cadre de transactions de pleine concurrence, une partie qui apporte des fonds mais ne contrôle pas les risques, ni n'exerce aucune autre fonction liée à l'activité financée, ne bénéficie généralement pas d'un rendement anticipé équivalent à celui d'un investisseur dont la situation est similaire mais qui exerce et contrôle également des fonctions importantes et assume et contrôle des risques importants associés à l'activité financée. La nature et le montant de la rémunération attribuable à une entité qui assume uniquement des coûts liés à des actifs incorporels doit être déterminée sur la base de tous les faits pertinents, et doit correspondre à la rémunération qui serait versée dans le cadre de montages financiers similaires entre des entités indépendantes sous réserve que de tels montages puissent être identifiés.

6.60 Le financement et la prise de risques sont intrinsèquement liés au sens où le financement coïncide souvent avec la prise de certains risques (ainsi, une partie qui apporte des fonds court souvent le risque de les perdre). Néanmoins, ils peuvent et doivent être analysés séparément, car il n'existe pas d'ensemble standard de risques assumés lors du financement de la mise au point, de l'amélioration, de l'entretien, de la protection ou de l'exploitation d'un actif incorporel. En fait, le risque assumé varie en fonction des dispositions contractuelles, du comportement et de la solvabilité des membres du groupe concernés, ainsi que de la nature du contrôle qu'ils exercent sur les risques assumés, entre autres facteurs. Le moment auquel intervient le financement au cours du processus de mise au point d'un actif incorporel peut également influer sur le niveau de risque. Ainsi, le fait qu'un investissement soit réalisé à un stade précoce, à miparcours du processus de mise au point ou à un stade avancé a un impact sur 
le niveau du risque d'investissement sous-jacent. La nature et l'ampleur du risque assumé doivent donc être déterminées sur la base de tous les faits et circonstances des relations existant entre les membres du groupe multinational concernés. Il ne peut être simplement supposé ou affirmé que la partie qui finance la mise au point d'un actif incorporel assume tous les risques associés à cette mise au point.

6.61 Si l'on pose l'hypothèse qu'il ne prend en charge aucun autre risque, un bailleur de fonds assumant un risque de financement est généralement en droit de bénéficier à ce titre d'un taux de rendement anticipé corrigé des risques, mais pas davantage. Les principales questions qui se posent dans chaque cas sont les suivantes: (i) quel est le risque financier assumé par l'entité qui apporte des fonds, (ii) a-t-elle la capacité financière d'assumer ce risque, (iii) comment et par qui ce risque financier est-il contrôlé (au sens des paragraphes 9.22 à 9.28), (iv) quelles sont les options de financement réalistes à la disposition des parties, et (v) quelle est la rémunération de pleine concurrence anticipée au titre de la prise en charge du risque financier en question?

\section{Prise en charge de risques}

6.62 Pour déterminer la répartition des risques liés aux actifs incorporels entre les membres d'un groupe multinational, il convient d'appliquer les principes énoncés aux paragraphes 9.10 à 9.46. Il faut notamment prendre en compte les dispositions du paragraphe 9.18 concernant l'importance des données mettant en évidence une répartition des risques similaire dans des transactions comparables sur le marché libre, les considérations sur le contrôle des risques figurant aux paragraphes 9.23 à 9.28 , les considérations concernant la capacité financière d'assumer le risque exposées aux paragraphes 9.29 à 9.32, ainsi que les dispositions du paragraphe 9.38 pour évaluer l'incidence des risques liés à la mise au point, à l'amélioration, à l'entretien, à la protection et à l'exploitation d'actifs incorporels.

6.63 L'identité du membre (ou des membres) du groupe multinational considéré qui assume et contrôle les risques liés à la mise au point, à l'amélioration, à l'entretien, à la protection et à l'exploitation d'actifs incorporels constitue également un élément important à prendre en compte pour déterminer les prix de transactions contrôlées et établir quelle entité (ou entités) sera en droit de bénéficier d'une partie des revenus tirés de l'exploitation de ces actifs incorporels. Le propriétaire légal peut assumer et contrôler ces risques. Si toutefois ce sont d'autres membres du groupe qui assument ou contrôlent les risques en question, ces membres doivent être rémunérés pour leur contribution, y compris les risques qu'ils prennent en 
charge. Cela peut signifier, par exemple, que ces parties se voient attribuer les revenus anticipés restants après que le propriétaire légal a été rémunéré pour la valeur anticipée des fonctions exercées, des actifs utilisés et des risques assumés (le cas échéant) par lui-même, et que les contribution des autres membres du groupe multinational ont également été rémunérées de manière similaire.

6.64 Les types de risques particuliers pouvant avoir une importance dans le cadre d'une analyse fonctionnelle relative à des transactions portant sur des actifs incorporels sont (i) les risques liés à la mise au point d'actifs incorporels, notamment le risque que des activités coûteuses de recherchedéveloppement ou de commercialisation ne débouchent sur un échec; (ii) le risque d'obsolescence des produits, notamment la possibilité que les progrès technologiques réalisés par des concurrents aient un effet préjudiciable sur la valeur des actifs incorporels considérés ; (iii) le risque d'atteinte aux droits sur des actifs incorporels, notamment le risque que la défense des droits sur les actifs incorporels considérés ou la défense contre des allégations d'atteintes émanant de tierces parties ne s'avère chronophage, coûteuse et/ou infructueuse ; et (iv) les risques découlant de la responsabilité du fait des produits et risques similaires liés aux biens et services fondés sur les actifs incorporels considérés. L'existence et le niveau de ces risques dépend des faits et circonstances de chaque cas d'espèce, ainsi que de la nature de l'actif incorporel concerné.

6.65 Il est particulièrement important de s'assurer que le membre (ou les membres) du groupe multinational considéré qui prétend, en vertu de dispositions contractuelles, à des revenus au titre de la prise en charge de risques assume effectivement la responsabilité des coûts à supporter en cas de matérialisation des risques considérés. Lorsqu'une partie à une transaction se voit attribuer des risques par contrat et exerce les fonctions de contrôle de ces risques, tandis que l'autre partie supporte les coûts découlant de ces risques, un ajustement de prix de transfert peut être nécessaire pour tenir compte du partage effectif des risques et répartir de manière appropriée les coûts correspondants. L'exemple 8 qui figure à l'annexe du chapitre VI illustre ce principe.

\section{(d) Revenus ex post imprévus}

6.66 Il est assez courant que des événements imprévus débouchent sur une augmentation ou une diminution des revenus tirés de l'exploitation d'un actif incorporel, de sorte que sa rentabilité effective (ex post) diffère de sa rentabilité anticipée (ex ante). Ainsi, il peut arriver qu'un produit compétitif soit retiré du marché, qu'une catastrophe naturelle ait lieu sur un marché essentiel, qu'un actif essentiel dysfonctionne pour des raisons imprévues, ou 
qu'une percée technologique de la part d'un concurrent ait pour effet de rendre obsolètes ou moins désirables les produits fondés sur l'actif incorporel considéré. Il peut également arriver que des prévisions financières, sur lesquelles reposent les calculs des revenus ex ante et les dispositions de rémunération, se révèlent tout simplement inexactes. Dans ces circonstances, la question se pose de savoir si, et si oui comment, les bénéfices ou pertes liés à ces événements imprévus doivent être répartis entre les membres d'un groupe multinational ayant contribué à la mise au point, à l'amélioration, à l'entretien, à la protection et à l'exploitation de l'actif incorporel considéré.

6.67 Pour répondre à cette question, il faut procéder à une analyse minutieuse visant à déterminer si les revenus sont en fait attribuables aux événements imprévus, et quelle entité (ou entités) du groupe multinational assume en fait les risques de ces événements imprévus. L'entité (ou les entités) qui a droit aux avantages ou est tenue d'assumer les charges découlant de ces événements imprévus peut être ou non le propriétaire légal de l'actif incorporel considéré, et peut être ou non l'entité qui finance le développement, l'amélioration, l'entretien, la protection ou l'exploitation de cet actif. Les conditions contractuelles constituent le point de départ de cette analyse. Néanmoins, une analyse minutieuse de deux autres points est également nécessaire. En premier lieu, il faut se demander si la rémunération ex ante versée aux membres du groupe multinational pour les fonctions qu'ils exercent, les actifs qu'ils utilisent et les risques qu'ils assument est conforme au comportement des parties et au principe de pleine concurrence. Il convient également de vérifier, par exemple, si le groupe a en fait minoré les bénéfices anticipés, entraînant le versement de paiements insuffisants (déterminés sur une base ex ante) à certains membres du groupe au titre de leur contribution. En second lieu, il faut se demander quelle entité (ou quelles entités) du groupe multinational considéré assume le risque de ces événements imprévus. À cet égard, il convient d'analyser la situation afin de déterminer quelle entité (ou quelles entités) exercent des fonctions liées au risque d'événements imprévus. Il faut notamment prendre en considération les fonctions liées au contrôle et à la gestion des risques concernés, notamment l'élaboration et la mise en œuvre des stratégies d'atténuation des risques. Ces fonctions recouvrent celles liées à la surveillance et à la gestion (si possible) des circonstances débouchant sur de tels événements imprévus, la gestion et le contrôle de fonctions et actifs spécifiques si les risques sont liés à ces fonctions et actifs spécifiques, ainsi que le contrôle et la gestion des conséquences des événements imprévus sur le plan financier et en termes de notoriété. L'évaluation de la capacité financière de diverses entités d'assumer les risques liés aux événements imprévus est également importante. Dans certaines situations, il peut être 
établi que, compte tenu des faits du cas d'espèce, la répartition contractuelle des risques ne correspond pas au comportement des parties, ou que les dispositions prises par le contribuable ne doivent pas être prises en compte ou être requalifiées conformément aux principes énoncés aux paragraphes 1.64 à 1.69. Il convient d'appliquer les principes exposés dans les chapitres I à III, dans le chapitre IX et dans le présent chapitre pour résoudre ces problèmes.

\section{Interactions avec la poursuite des travaux sur l'érosion de la base d'imposition et le transfert de bénéfices}

En théorie, si la rémunération ex ante de tous les membres d'un groupe multinational au titre de leur contribution est véritablement déterminée suivant le principe de pleine concurrence, et si les dispositions du paragraphe 6.67 sont appliquées, les bénéfices imprévus devraient tendre à suivre un " trajet aléatoire ": autrement dit, on observerait parfois des bénéfices imprévus, parfois des écarts négatifs imprévus par rapport aux bénéfices anticipés, voire des pertes, et la répartition contractuelle de ces bénéfices ou pertes imprévus entre les membres du groupe multinational considéré, quels qu'ils soient, ne correspondrait pas à un avantage anticipé systématiquement corrigé des risques. Malheureusement, dans certaines circonstances, les revenus effectifs peuvent être systématiquement supérieurs aux revenus anticipés d'une façon laissant à penser que les contribuables minorent systématiquement ces revenus anticipés, de sorte que les revenus effectivement perçus par le propriétaire légal des actifs incorporels considérés sont systématiquement supérieurs à ceux qui étaient anticipés. Il est clair que dans certaines situations, les contribuables sont incités à surévaluer le risque anticipé et à minorer la rémunération appropriée devant être versée par le propriétaire légal aux autres membres du groupe multinational considéré. Les contribuables ont également la possibilité pratique de manipuler ainsi les prix en exploitant les asymétries d'information qui existent entre le contribuable et les administrations fiscales, et le passage du temps entre le moment où les prix ont été initialement déterminés et celui où les vérifications ont lieu. $\mathrm{La}$ section D.3 fournit des instructions concernant les situations, telles que celles évoquées précédemment, dans lesquelles l'évaluation peut être très incertaine au moment de la transaction. L'expérience a montré dans les pays G20 et de l'OCDE que ces situations et scénarios sont tout particulièrement susceptibles de se traduire par des phénomènes d'érosion de la base d'imposition et de transfert de bénéfices. 
(e) Résumé des sections B.1 et B.2

6.68 En substance, si le propriétaire légal d'un actif incorporel :

- $\quad$ exerce et contrôle toutes les fonctions (y compris les fonctions importantes décrites au paragraphe 6.56) liées à la mise au point, à l'amélioration, à l'entretien, à la protection et à l'exploitation de cet actif incorporel ;

- fournit tous les actifs, y compris les fonds, nécessaires à la mise au point, à l'amélioration, à l'entretien, à la protection et à l'exploitation de l'actif incorporel considéré ; et

- assume et contrôle tous les risques liés à la mise au point, à l'amélioration, à l'entretien, à la protection et à l'exploitation de l'actif incorporel en question,

il est en droit de recevoir la totalité des revenus anticipés, ex ante, qui devraient être tirés par le groupe multinational concerné de l'exploitation de cet actif incorporel. Pour autant qu'un ou plusieurs membres de ce groupe multinational autres que le propriétaire légal exercent des fonctions, utilisent des actifs ou assument des risques liés à la mise au point, à l'amélioration, à l'entretien, à la protection ou à l'exploitation de l'actif incorporel considéré, ces entreprises associées doivent obtenir une partie des revenus anticipés susceptibles d'être tirés de l'exploitation de cet actif incorporel, en recevant une rémunération de pleine concurrence au titre des fonctions qu'ils ont exercées, des actifs qu'ils ont utilisés et des risques qu'ils ont assumés. Cette rémunération peut, suivant les faits et circonstances, constituer la totalité ou une part importante des revenus escomptés de l'exploitation de l'actif incorporel considéré.

6.69 Le droit d'un membre du groupe multinational concerné aux bénéfices (ou pertes, ex post) liés à des événements imprévus dépendra des conditions des contrats pertinents ainsi que des fonctions exercées, des actifs utilisés et des risques assumés en relation avec ces événements imprévus.]

\section{B.3. Identifier et déterminer les prix et autres conditions des transactions contrôlées}

6.70 La réalisation de l'analyse de comparabilité décrite dans les chapitres I à III, complétée par le présent chapitre, devrait faciliter une évaluation claire de la propriété légale des actifs incorporels ainsi que des fonctions, actifs et risques associés à ces actifs, et une identification précise des transactions dont les prix et autres conditions doivent être déterminés. 
En règle générale, les transactions identifiées par le groupe multinational considéré dans les documents d'immatriculation et contrats pertinents sont celles dont les prix et autres conditions doivent être déterminés suivant le principe de pleine concurrence. Néanmoins, l'analyse de comparabilité peut révéler que des transactions s'ajoutant aux transactions décrites dans les documents d'immatriculation et contrats, ou différentes de ces transactions, ont en fait eu lieu. Conformément au paragraphe 1.53, les transactions (et leurs véritables conditions) devant être analysées sont celles dont il est établi qu'elles ont eu lieu sur la base du comportement effectif des parties et des autres faits pertinents.

6.71 Il convient de déterminer les prix de pleine concurrence et les autres conditions des transactions suivant les instructions qui figurent aux chapitres I à III, en tenant compte des contributions à la valeur incorporelle anticipée des fonctions exercées, des actifs utilisés et des risques assumés au moment où ces fonctions sont exercées, ces actifs utilisés et ces risques assumés, comme indiqué dans la section $\mathrm{B}$. La section $\mathrm{D}$ fournit des instructions supplémentaires sur les méthodes d'établissement des prix de transfert et autres dispositions applicables pour déterminer les prix de pleine concurrence et autres conditions des transactions portant sur des actifs incorporels.

\section{B.4. Application des principes précédents à des scénarios spécifiques}

6.72 Les principes énoncés dans cette section B doivent être appliqués dans diverses situations liées au développement, à l'amélioration, à l'entretien, à la protection et à l'exploitation d'actifs incorporels. Une considération essentielle dans chaque cas est que les entreprises associées contribuant à la mise au point, à l'amélioration, à l'entretien, à la protection ou à l'exploitation d'actifs incorporels dont un autre membre du groupe multinational considéré est le propriétaire légal doivent recevoir une rémunération de pleine concurrence au titre des fonctions qu'ils exercent, des risques qu'ils assument et des actifs qu'ils utilisent. Pour déterminer si les entreprises associées qui exercent des fonctions ou assument des risques liés à la mise au point, à l'amélioration, à l'entretien, à la protection et à l'exploitation d'actifs incorporels ont reçu en conséquence une rémunération de pleine concurrence, il est nécessaire d'examiner (i) l'ampleur et la nature des activités entreprises et (ii) le montant et la forme de la rémunération payée. Pour déterminer si la rémunération fournie dans le cadre de la transaction contrôlée est conforme au principe de pleine concurrence, il faut se référer à l'ampleur et à la nature des activités d'entités indépendantes comparables exerçant des fonctions similaires, à la rémunération reçue par des entités indépendantes comparables exerçant des fonctions similaires, et à la création anticipée de valeur incorporelle par des entités indépendantes 
comparables exerçant des fonctions similaires. Cette section décrit l'application de ces principes dans des scénarios courants.

(a) Développement et amélioration d'actifs incorporels de commercialisation

6.73 Une situation courante dans laquelle ces principes doivent être appliqués se présente lorsqu'une entreprise associée avec le propriétaire légal d'une marque de fabrique exerce des fonctions de commercialisation ou de vente dont bénéficie le propriétaire légal de la marque en question, par exemple dans le cadre d'un accord de commercialisation ou d'un accord de distribution/commercialisation. Dans ce cas de figure, il est nécessaire de déterminer comment l'entité chargée de la commercialisation ou de la distribution doit être rémunérée pour ses activités. Une question importante est de savoir si l'entité chargée de la commercialisation/distribution doit être rémunérée uniquement pour avoir fourni des services de promotion et de distribution, ou si elle doit également être rémunérée pour avoir accru la valeur de la marque de fabrique considérée et d'autres actifs incorporels de commercialisation grâce aux fonctions qu'elle a exercées, aux actifs qu'elle a utilisés et aux risques qu'elle a assumés.

6.74 L'analyse de ce point passe par une évaluation (i) des obligations et droits découlant des enregistrements légaux et des accords conclus entre les parties; (ii) des fonctions exercées, des actifs utilisés et des risques assumés par les parties; (iii) de la création de valeur incorporelle anticipée qui devrait résulter des activités de l'entité chargée de la commercialisation/distribution; et (iv) de la rémunération fournie pour les fonctions exercées par l'entité chargée de la commercialisation/distribution (compte tenu des actifs utilisés et des risques assumés). La situation est relativement claire lorsqu'un distributeur intervient uniquement en tant qu'agent, le propriétaire de la marque de fabrique et des autres actifs incorporels de commercialisation considérés lui remboursant ses dépenses promotionnelles tout en dirigeant et contrôlant ses activités. Dans ce cas, le distributeur a généralement droit uniquement à une rémunération correspondant à ses activités d'agent. Il n'assume pas ni ne contrôle les risques associés à la poursuite de la valorisation de la marque de fabrique et des autres actifs incorporels de commercialisation considérés, et il n'aura donc droit à aucune rémunération complémentaire à cet égard.

6.75 Lorsque le distributeur supporte effectivement le coût de ses activités de commercialisation (par exemple en l'absence de toute disposition prévoyant le remboursement des dépenses en cause par le propriétaire légal), l'analyse doit viser à déterminer dans quelle mesure le 
distributeur peut bénéficier d'une fraction des avantages potentiels découlant des fonctions exercées, des actifs utilisés et des risques assumés actuellement ou dans l'avenir par ce distributeur. En règle générale, dans le cadre de transactions de pleine concurrence, la capacité d'une partie qui n'est pas le propriétaire légal d'une marque de fabrique et d'autres actifs incorporels de commercialisation de bénéficier des avantages découlant d'activités de commercialisation qui accroissent la valeur de ces actifs incorporels sera principalement fonction de la nature des droits de cette partie. Par exemple, un distributeur pourra bénéficier des avantages découlant des fonctions exercées, des actifs utilisés et des risques assumés par lui pour renforcer la valeur d'une marque de fabrique et d'autres actifs incorporels de commercialisation, en termes de chiffre d'affaires et de part de marché, s'il a conclu un contrat à long terme de distribution exclusive du produit de marque considéré. Dans une telle situation, il est possible que les efforts déployés par le distributeur aient accru la valeur de ses propres actifs incorporels, à savoir ses droits de distribution. Il convient alors de déterminer la part des avantages considérés revenant au distributeur sur la base de ce qu'un distributeur indépendant obtiendrait dans des circonstances comparables. Dans certains cas, un distributeur peut exercer des fonctions, utiliser des actifs ou assumer des risques allant au-delà de ce qu'un distributeur indépendant ayant des droits similaires pourrait supporter ou réaliser pour ses propres activités de distribution, et qui créent une valeur supérieure à celle créée par d'autres entités de commercialisation/distribution en situation similaire. En pareil cas, un distributeur indépendant demandera généralement une rémunération supplémentaire au propriétaire de la marque de fabrique ou des autres actifs incorporels considérés. Une telle rémunération peut prendre la forme d'une hausse des bénéfices tirés des activités de distribution (résultant d'une diminution du prix d'achat du produit considéré), d'une réduction du taux de la redevance, ou d'une part des bénéfices liés à l'augmentation de la valeur de marque de fabrique et des autres actifs incorporels considérés, l'objectif étant de rémunérer le distributeur au titre de ses fonctions, actifs et risques, et de la création de valeur anticipée. Les exemples 9 à 14 figurant dans l'annexe au chapitre VI illustrent de façon plus détaillée l'application de la présente section B dans le contexte des accords de commercialisation et de distribution.

(b) Dispositions relatives à la recherche-développement et à l'amélioration des procédés

6.76 Les principes énoncés dans les paragraphes précédents s'appliquent également aux situations dans lesquelles des fonctions de recherche-développement sont exercées par un membre d'un groupe multinational en vertu de dispositions contractuelles conclues avec une 
entreprise associée qui est le propriétaire légal des actifs incorporels pouvant résulter de ces activités. La rémunération appropriée des services de recherche dépendra de tous les faits et circonstances du cas d'espèce, notamment du fait de savoir si l'équipe de recherche possède une expérience et des qualifications sans équivalent au regard des activités de recherche considérées, si elle assume des risques (compte tenu, par exemple, du lieu où est réalisée la recherche fondamentale), si elle utilise ses propres actifs incorporels, ou si elle est contrôlée et gérée par une autre partie. Une rémunération égale au remboursement des dépenses augmenté d'une marge modeste ne correspondra pas à la valeur anticipée ni au prix de pleine concurrence des contributions de cette équipe de recherche dans tous les cas de figure.

6.77 Les principes énoncés dans la présente section s'appliquent également aux situations dans lesquelles un membre d'un groupe multinational fournit des services de fabrication susceptibles de déboucher sur des améliorations de procédé ou de produit au nom d'une entreprise associée qui assumera la propriété légale de ces améliorations de procédé ou de produit. Les exemples 15 à 18 qui figurent dans l'annexe au chapitre VI illustrent de manière plus détaillée l'application de la présente section $\mathrm{B}$ dans le contexte des dispositions relatives à la recherche-développement.

6.78 Des questions se posent souvent concernant la rémunération de pleine concurrence à verser au titre de l'utilisation des noms de groupe, noms commerciaux et autres actifs incorporels similaires. Il convient de trancher ces questions sur la base des principes énoncés dans la présente section $\mathrm{B}$ et des facteurs commerciaux et juridiques en jeu. De manière générale, aucun paiement ne sera reconnu aux fins de la détermination de prix de transfert au titre de la simple prise en compte de l'appartenance à un groupe multinational, ou d'une utilisation du nom du groupe considéré uniquement destinée à refléter l'appartenance à ce groupe. Voir le paragraphe 7.13 .

6.79 Dès lors qu'un membre du groupe multinational considéré est le propriétaire d'une marque de fabrique ou d'un autre actif incorporel au nom du groupe, et que l'utilisation de ce nom confère un avantage financier à des membres du groupe autres que celui qui est légalement propriétaire de l'actif incorporel considéré, il est raisonnable d'en conclure que cette utilisation aurait donné lieu à un paiement dans le cadre d'une transaction de pleine concurrence. De même, un tel paiement peut être approprié dès lors qu'un membre du groupe possède des éléments de survaleur concernant une activité représentée par une marque de fabrique non déposée, que 
l'utilisation de cette marque de fabrique par une autre partie constituerait une représentation trompeuse, et que l'utilisation de cette marque de fabrique procure un avantage financier évident à un membre du groupe autre que celui qui possède les éléments de survaleur et la marque de fabrique non déposés en question.

6.80 Pour déterminer le montant du paiement relatif à un nom de groupe, il importe de prendre en considération le montant de l'avantage financier que représente l'utilisation de ce nom pour son utilisateur, les coûts et avantages associés à d'autres options, et les contributions relatives à la valeur de ce nom du propriétaire légal, d'une part, et de l'entité utilisant ce nom, d'autre part, sous la forme de fonctions exercées, d'actifs utilisés et de risques assumés. Il convient d'examiner avec soin les fonctions exercées, les actifs utilisés et les risques assumés par l'utilisateur du nom considéré qui concourent à créer ou accroître la valeur de ce nom dans sa juridiction. Les facteurs qui joueraient un rôle important dans le cadre d'une concession sous licence de ce nom à une entreprise indépendante dans des circonstances comparables, en application des principes énoncées dans les chapitres I à III, doivent être pris en compte.

6.81 Dès lors qu'une entreprise existante et florissante est acquise par une autre entreprise, et que la première commence à utiliser un nom, une marque de fabrique ou un autre élément de marque faisant référence à la seconde, il ne faut pas partir du principe que cette utilisation doit donner lieu à un paiement. Si l'on peut raisonnablement s'attendre à ce que l'entreprise achetée tire un avantage financier de l'utilisation de la marque de l'entreprise acquéreuse, le montant d'un éventuel paiement doit être déterminé en fonction du niveau de cet avantage anticipé.

6.82 Il peut également arriver que l'entreprise acquéreuse mette à profit la position occupée par l'entreprise achetée pour développer ses propres activités sur le territoire où opère cette dernière, en l'amenant à utiliser sa marque. Dans ce cas, il convient de se demander si l'acquéreur doit effectuer un paiement à l'entreprise achetée, ou la rémunérer de quelque autre manière que ce soit, pour les fonctions exercées, les risques assumés et les actifs utilisés (y compris sa position sur le marché) en rapport avec l'utilisation accrue du nom de l'acquéreur. 


\section{Transactions portant sur l'utilisation ou le transfert d'actifs incorporels}

6.83 Aux fins de la détermination des prix de transfert, il est non seulement nécessaire d'identifier avec précision les actifs incorporels concernés dans chaque cas d'espèce, et d'identifier le propriétaire de ces actifs, mais il faut aussi identifier et qualifier correctement, au début de toute analyse de prix de transfert portant sur des actifs incorporels, les transactions contrôlées qui ont spécifiquement trait à ces actifs. Il convient d'appliquer les principes du chapitre I pour identifier et qualifier les transactions se rapportant à l'utilisation ou au transfert d'actifs incorporels. Outre les instructions relatives aux dispositions contractuelles (paragraphe 1.53) et aux réorganisations d'entreprises (chapitre IX, en particulier la partie II), la section $\mathrm{C}$ décrit des scénarios typiques qui peuvent être utiles pour déterminer si une transaction fait intervenir des actifs incorporels ou des droits sur des actifs incorporels. Voir l'exemple 20. La qualification d'une transaction aux fins de la détermination de prix de transfert est sans conséquence pour les éléments devant être déterminés en vertu de l'article 12 du Modèle de Convention fiscale de l'OCDE. Voir par exemple les paragraphes 8 à 19 du Commentaire de l'article 12 du Modèle de Convention fiscale de l'OCDE.

6.84 Il existe deux grandes catégories de transactions pour lesquelles l'identification et l'examen des actifs incorporels seront utiles aux fins de la détermination de prix de transfert. Il s'agit (i) des transactions portant sur le transfert d'actifs incorporels ou de droits sur des actifs incorporels et (ii) des transactions portant sur l'utilisation d'actifs incorporels en relation avec la vente de biens ou la fourniture de services.

\section{C.1. Transactions portant sur le transfert d'actifs incorporels ou de droits sur des actifs incorporels}

\section{(i) Transfert d'actifs incorporels ou de droits sur des actifs incorporels}

6.85 Les droits sur des actifs incorporels eux-mêmes peuvent être transférés dans le cadre de transactions contrôlées. Ces transactions peuvent porter sur le transfert de tous les droits sur l'actif incorporel considéré (par exemple, une vente de cet actif incorporel ou la concession d'une licence exclusive d'utilisation perpétuelle de l'actif en question) ou de certains droits uniquement (par exemple, une concession de licence ou un transfert similaire de droits limités d'utilisation d'un actif incorporel, pouvant faire l'objet de restrictions géographiques, d'une limitation de durée ou de 
restrictions relatives au droit d'utiliser cet actif, de l'exploiter, de le reproduire, de le transférer à nouveau ou de poursuivre sa mise au point). Il convient d'appliquer les principes énoncés aux chapitres I à III aux transactions portant sur le transfert d'actifs incorporels ou de droits sur des actifs incorporels. Des instructions complémentaires concernant la détermination des conditions de pleine concurrence pour ce type de transactions figurent également dans les sections D.1, D.2 et D.3 du présent chapitre.

6.86 Dans le cadre des transactions portant sur le transfert d'actifs incorporels ou de droits sur des actifs incorporels, il est essentiel d'identifier avec précision la nature des actifs incorporels ou des droits sur des actifs incorporels qui sont transférés entre des entreprises associées. Lorsque les droits transférés font l'objet de limitations, il est également essentiel de déterminer la nature de ces limitations et toute l'étendue des droits transférés. Il convient de noter à cet égard que les qualifications retenues pour les transactions ne sont aucunement déterminantes pour l'analyse des prix de transfert. Ainsi, dans le cas d'un transfert du droit exclusif d'exploitation d'un brevet dans le pays X, la décision du contribuable de qualifier cette transaction soit de vente de la totalité des droits de brevet dans le pays $\mathrm{X}$, soit de concession de licence exclusive d'utilisation perpétuelle d'une partie des droits de licence mondiaux, n'a pas d'incidence sur la détermination du prix de pleine concurrence si, dans l'un ou l'autre cas, la transaction dont le prix est établi est un transfert de droits exclusifs d'exploitation du brevet dans le pays $\mathrm{X}$ au cours de sa durée d'utilité résiduelle. Par conséquent, l'analyse fonctionnelle doit permettre de déterminer avec précision la nature des droits transférés sur des actifs incorporels.

6.87 Les restrictions imposées dans le cadre d'accords de licence ou d'accords similaires concernant l'utilisation d'un actif incorporel pour la mise au point de nouveaux actifs incorporels ou de nouveaux produits faisant appel à cet actif incorporel revêtent souvent une importance considérable dans une analyse de prix de transfert. Il importe donc, pour déterminer la nature d'un transfert de droits sur des actifs incorporels, de se demander si le cessionnaire reçoit le droit d'utiliser l'actif incorporel transféré aux fins de nouvelles activités de recherche-développement. Dans le cadre de transactions entre des entreprises indépendantes, il arrive qu'en vertu des dispositions adoptées, le cédant/donneur de licence conserve l'intégralité des droits sur les éventuelles améliorations de l'actif incorporel concédé sous licence qui pourraient avoir lieu au cours de la durée de la licence. On observe également des transactions entre entreprises indépendantes dans le cadre desquelles le cessionnaire/preneur de licence conserve les droits sur 
les éventuelles améliorations qu'il est susceptible de réaliser, soit pour la durée de la licence, soit de manière perpétuelle. La nature des éventuelles limitations concernant la poursuite de la mise au point des actifs incorporels transférés, ou la capacité du cessionnaire et du cédant de tirer un avantage économique de telles améliorations, peut avoir une incidence sur la valeur des droits transférés et la comparabilité de deux transactions portant par ailleurs sur des actifs incorporels identiques ou étroitement comparables. Ces limitations doivent être évaluées à la fois à la lumière des dispositions écrites des accords conclus et du comportement effectif des parties concernées.

6.88 Il convient d'appliquer les dispositions des paragraphes 1.52 à 1.54 ainsi que celles des paragraphes 1.64 à 1.69 pour déterminer la nature exacte d'une transaction portant sur un transfert d'actifs incorporels ou de droits sur des actifs incorporels, pour déterminer la nature des actifs incorporels éventuellement transférés, et pour identifier les éventuelles limitations imposées par les conditions du transfert concernant l'utilisation des actifs incorporels en question. Ainsi, en vertu des paragraphes 1.52 à 1.54, même s'il est spécifié par écrit qu'une licence est non exclusive ou d'une durée limitée, l'administration fiscale ne devra pas nécessairement respecter cette spécification si celle-ci ne concorde pas avec le comportement des parties. L'exemple 19 qui figure à l'annexe du chapitre VI illustre les dispositions de ce paragraphe.

\section{(ii) Transferts de combinaisons d'actifs incorporels}

6.89 Des actifs incorporels (y compris des droits limités portant sur des actifs incorporels) peuvent être transférés séparément ou en combinaison avec d'autres actifs incorporels. L'examen des transactions portant sur le transfert d'une combinaison d'actifs incorporels soulève souvent deux questions liées.

6.90 La première porte sur la nature et les conséquences économiques des interactions entre différents actifs incorporels. Il peut arriver que certains actifs incorporels aient davantage de valeur en combinaison avec d'autres actifs incorporels que séparément. Il importe donc de déterminer la nature des interactions juridiques et économiques entre les actifs incorporels qui sont transférés conjointement.

6.91 Ainsi, un produit pharmaceutique est souvent associé à trois types d'actifs incorporels, voire davantage. Le principe actif peut être protégé par un ou plusieurs brevets. Ce produit a également fait l'objet de procédures d'essai, et un organisme public de réglementation a pu délivrer une autorisation de mise sur le marché du produit en question sur un marché 
géographique donné et pour des indications approuvées spécifiques, sur la base des essais réalisés. Le produit peut être commercialisé sous une marque de fabrique particulière. Combinés, ces actifs incorporels peuvent avoir une valeur extrêmement élevée. Isolément, un de ces actifs ou plusieurs peuvent avoir une valeur nettement plus faible. Ainsi, la marque de fabrique sans le brevet et l'autorisation réglementaire de mise sur le marché peut avoir une valeur limitée, étant donné que le produit considéré ne pourrait être vendu sans l'autorisation de mise sur le marché, et que des produits génériques concurrents ne pourraient être exclus du marché en l'absence du brevet. De même, la valeur du brevet peut être nettement plus élevée après l'obtention d'une autorisation réglementaire de mise sur le marché qu'en l'absence de cette autorisation. Les interactions entre ces actifs de catégories différentes, ainsi que la répartition des rôles entre les parties s'agissant de l'exécution des fonctions, de la prise en charge des risques et du financement des coûts associés à la protection des actifs incorporels considérés, constituent donc des éléments très importants à prendre en compte dans une analyse de prix de transfert concernant un transfert de ces actifs incorporels. Lorsque différentes entreprises associées détiennent des droits sur les actifs incorporels utilisés, il importe d'examiner leurs contributions relatives en termes de création de valeur.

6.92 La seconde question, liée à la première, tient à l'importance de veiller à ce que tous les actifs incorporels transférés dans le cadre d'une transaction particulière aient été identifiés. Il peut arriver, par exemple, que des actifs incorporels soient tellement interdépendants qu'il est fondamentalement impossible de transférer l'un sans transférer l'autre. De fait, il est fréquent que le transfert d'un actif incorporel passe nécessairement par celui d'autres actifs incorporels. Dans ce cas, il importe d'identifier tous les actifs incorporels mis à la disposition du cessionnaire en conséquence d'un transfert d'actifs incorporels, en appliquant les principes énoncés aux paragraphes 1.52 à 1.54. Ainsi, le transfert de droits d'utilisation d'une marque de fabrique dans le cadre d'un accord de licence s'accompagne généralement de la concession sous licence de la valeur de notoriété, parfois qualifiée de survaleur, associée à cette marque de fabrique, sachant que cette survaleur a été créée par le cédant. Tout droit de licence exigé devra être déterminé à la fois sur la base de la marque de fabrique et de la valeur de notoriété qui lui est associée. L'exemple 21 qui figure à l'annexe du chapitre VI illustre les principes énoncés dans ce paragraphe.

6.93 Il importe d'identifier les situations dans lesquelles des contribuables ou des administrations fiscales peuvent tenter de dissocier artificiellement des actifs incorporels qui, pour des raisons de fond, ne seraient pas dissociées par des parties indépendantes dans des circonstances 
comparables. Ainsi, il convient d'identifier les tentatives de dissocier artificiellement une marque de fabrique ou un nom commercial de la survaleur ou de la valeur de notoriété qui est de fait associée à cette marque de fabrique ou à ce nom commercial, et de procéder à une analyse critique de ces tentatives. L'exemple 22 qui figure à l'annexe du chapitre VI illustre les principes énoncés dans ce paragraphe.

6.94 Il convient de reconnaître que le processus d'identification de tous les actifs incorporels transférés dans le cadre d'une transaction particulière consiste à identifier, en se fondant sur les accords écrits pertinents et le comportement effectif des parties, les transactions effectives ayant eu lieu, en appliquant les principes énoncés aux paragraphes 1.52 à 1.54 .

(iii) Transferts d'actifs incorporels ou de droits sur des actifs incorporels combinés à d'autres transactions commerciales

6.95 Dans certaines situations, des actifs incorporels ou des droits sur des actifs incorporels peuvent être transférés avec des actifs industriels ou commerciaux, ou avec des services. Il importe alors de déterminer si des actifs incorporels ont effectivement été transférés en relation avec la transaction considérée. Il importe également que tous les actifs incorporels transférés en relation avec une transaction particulière soient identifiés et pris en compte lors de l'analyse des prix de transfert. Les exemples 24 à 26 qui figurent dans l'annexe au chapitre VI illustrent les principes énoncés dans ce paragraphe.

6.96 Dans certaines situations, il peut s'avérer à la fois possible et approprié de dissocier les transactions relatives à des biens corporels ou des services des transferts d'actifs incorporels ou de droits sur des actifs incorporels aux fins de la réalisation d'une analyse des prix de transfert. Dans ces situations, il convient de décomposer le prix d'un contrat global pour confirmer que chaque élément de la transaction est conforme au principe de pleine concurrence. Dans d'autres situations, des transactions peuvent être si étroitement liées qu'il sera difficile de dissocier les transactions relatives à des biens corporels ou des services des transferts d'actifs incorporels ou de droits sur des actifs incorporels. La fiabilité des comparables disponibles constituera un facteur important à prendre en compte pour déterminer si des transactions doivent être combinées ou dissociées. Il importe en particulier de déterminer si les comparables disponibles permettent une évaluation précise des interactions entre les transactions considérées. 
6.97 L'existence d'un accord de franchise constitue une situation dans laquelle des transactions portant sur le transfert d'actifs incorporels ou de droits sur des actifs incorporels peuvent être combinées avec d'autres transactions. En vertu d'un accord de franchise, un membre d'un groupe multinational peut accepter de fournir une combinaison de services et d'actifs incorporels à une entreprise associée en contrepartie d'une redevance unique. Si les services et les actifs incorporels fournis dans le cadre d'un tel accord sont suffisamment spécifiques pour qu'il soit impossible d'identifier des comparables fiables pour l'ensemble constitué par les services et les actifs incorporels en question, il peut s'avérer nécessaire de dissocier les différents éléments de cet ensemble de services et d'actifs incorporels, pour les examiner séparément aux fins de la détermination des prix de transfert. Il convient cependant de garder à l'esprit que les interactions entre divers actifs incorporels et services peuvent accroitre leurs valeurs respectives.

6.98 Dans d'autres situations, la fourniture d'un service et le transfert d'un ou plusieurs actifs incorporels peuvent être si étroitement interdépendants qu'il est difficile de dissocier les transactions aux fins d'analyse des prix de transfert. Ainsi, certains transferts de droits sur des logiciels peuvent être combinés avec l'engagement du cédant de fournir des services de maintenance permanente des logiciels, qui peuvent inclure des mises à jour périodiques. Dans les situations où des services et des transferts d'actifs incorporels sont interdépendants, il peut être nécessaire de déterminer les prix de pleine concurrence sur une base globale.

6.99 Il convient de souligner que la qualification retenue pour la transaction considérée, à savoir fourniture de biens ou services, transfert d'actifs incorporels ou combinaison des deux, n'impose pas nécessairement l'utilisation d'une méthode particulière de détermination des prix de transfert. Ainsi, l'application de la méthode du coût majoré ne conviendra pas à toutes les transactions relatives à des services, et les transactions relatives à des actifs incorporels ne nécessitent pas toutes des évaluations complexes ou l'application de la méthode du partage des bénéfices. Les faits de chaque cas d'espèce, et les résultats de l'analyse fonctionnelle requise, détermineront la façon dont les transactions sont combinées, qualifiées et analysées aux fins de la détermination des prix de transfert, ainsi que la sélection de la méthode d'établissement des prix de transfert la plus appropriée au cas d'espèce. L'objectif visé in fine est de déterminer les prix et autres conditions qui seraient fixés entre des entreprises indépendantes dans le cadre de transactions comparables.

6.100 En outre, il convient également de souligner que pour déterminer si des transactions doivent être combinées ou dissociées aux fins d'analyse, il 
faut généralement cerner la transaction effectivement intervenue, sur la base des accords écrits ayant été conclus et du comportement effectif des parties concernées. Cela n'implique pas une requalification des transactions au sens des paragraphes 1.64 à 1.69. L'identification de la transaction effectivement intervenue constitue une condition nécessaire pour déterminer la méthode d'établissement des prix de transfert la plus appropriée au cas d'espèce.

\section{C.2. Transactions portant sur l'utilisation d'actifs incorporels en relation avec la vente de biens ou la fourniture de services}

6.101 Des actifs incorporels peuvent être utilisés en relation avec des transactions contrôlées dans des situations où il n'y a pas de transfert de l'actif incorporel considéré ni des droits sur cet actif incorporel. Ainsi, des actifs incorporels peuvent être utilisés par une des parties à une transaction contrôlée, ou les deux, en relation avec la fabrication de biens vendus à une entreprise associée, en relation avec la commercialisation de biens achetés à une entreprise associée, ou en relation avec la prestation de services au nom d'une entreprise associée. La nature d'une telle transaction doit être clairement spécifiée, et tout actif incorporel pertinent utilisé par l'une ou l'autre des parties en relation avec cette transaction contrôlée doit être identifié et pris en compte dans le cadre de l'analyse de comparabilité (notamment fonctionnelle), de la sélection et de l'application de la méthode de détermination des prix de transfert la plus appropriée à la transaction considérée, et du choix de la partie testée. Voir les paragraphes 1.39, 1.42, $1.44,2.110$ et 3.18. Des instructions supplémentaires concernant la détermination des conditions de pleine concurrence pour les transactions portant sur l'utilisation d'actifs incorporels en relation avec la vente de biens ou la prestation de services figurent dans les sections D.1 et D.4 du présent chapitre.

6.102 L'exemple suivant illustre la nécessité d'examiner l'utilisation d'actifs incorporels par une partie à une transaction contrôlée portant sur une vente de biens. Supposons qu'un constructeur automobile utilise des brevets exclusifs de valeur pour fabriquer des voitures, qu'il vend ensuite à des distributeurs associés. Supposons que ces brevets contribuent de manière importante à la valeur des véhicules automobiles en question. Ces brevets et leur contribution en termes de valeur doivent être pris en compte dans le cadre de l'analyse de comparabilité de la transaction consistant en la vente des voitures par le constructeur automobile à ses distributeurs associés, de la sélection de la méthode de détermination des prix de transfert la plus appropriée à cette transaction, et de la sélection de la partie testée. Les distributeurs associés qui achètent les voitures n'acquièrent cependant aucun droit sur les brevets du constructeur. Dans ce cas, les brevets sont utilisés 
dans le cadre de l'activité de fabrication et peuvent influer sur la valeur des véhicules automobiles, mais les brevets eux-mêmes ne sont pas transférés.

6.103 Un autre exemple permet d'illustrer l'utilisation d'actifs incorporels en relation avec une transaction contrôlée. Supposons qu'une société d'exploration ait acquis ou élaboré des données et analyses géologiques de valeur, ainsi qu'un savoir-faire et un logiciel d'exploration complexe. Supposons en outre qu'elle utilise ces actifs incorporels pour fournir des services d'exploration à une entreprise associée. Ces actifs incorporels doivent être pris en compte dans le cadre de l'analyse de comparabilité des transactions relatives à ces services entre la société d'exploration et l'entreprise associée, de la sélection de la méthode de détermination des prix de transfert la plus appropriée à la transaction considérée, et de la sélection de la partie testée. Si l'on suppose que l'entreprise associée à la société d'exploration n'acquiert aucun droit sur les actifs incorporels de ladite société, ces actifs incorporels sont utilisés pour la fourniture de services et peuvent influer sur la valeur de ces services, mais ils ne sont pas transférés. 


\section{Instructions supplémentaires pour déterminer les conditions de pleine concurrence dans le cas de transactions portant sur des actifs incorporels}

6.104 Après avoir identifié les transactions portant sur des actifs incorporels, en identifiant expressément les actifs concernés, et avoir déterminé la ou les entités légalement propriétaires de ces actifs, ainsi que les entités apportant une contribution à la valeur de ceux-ci, il devrait être possible de déterminer les conditions de pleine concurrence pour les transactions considérées. Les prescriptions énoncées aux chapitres I à III des présents Principes devraient être appliquées pour déterminer les conditions de pleine concurrence dans le cas de transactions portant sur des actifs incorporels. En particulier, le processus en neuf étapes décrit au paragraphe 3.4 peut s'avérer utile pour déterminer les conditions de pleine concurrence dans le cas de telles transactions. Afin de conduire une analyse de comparabilité conformément aux principes du chapitre III et au processus décrit au paragraphe 3.4 , il est essentiel de prendre en compte les principes exposés dans les sections $\mathrm{A}, \mathrm{B}$ et $\mathrm{C}$ du présent chapitre VI.

6.105 Les principes exposés dans les chapitres I à III peuvent toutefois se révéler difficiles à appliquer lorsque les transactions contrôlées portent sur des actifs incorporels. Ces actifs peuvent présenter un caractère spécifique compliquant la recherche de comparables et, parfois, la détermination de la valeur au moment de la transaction. En outre, dans certains cas, des entreprises associées, pour des raisons commerciales tout à fait légitimes tenant aux liens existant entre elles, peuvent réaliser des transactions portant sur des actifs incorporels selon des modalités que des entreprises indépendantes n'envisageraient pas (voir paragraphe 1.11). L'utilisation ou le transfert d'actifs incorporels peut soulever des problèmes complexes concernant la comparabilité, le choix des méthodes de prix de transfert et la détermination des conditions de pleine concurrence pour les transactions. La présente section D fournit des instructions supplémentaires concernant l'application des principes exposés dans les chapitres I - III pour déterminer les conditions de pleine concurrence dans le cas de transactions portant sur des actifs incorporels.

6.106 La section D.1 présente des instructions supplémentaires générales concernant toutes les transactions portant sur des actifs incorporels. La section D.2 expose des instructions supplémentaires particulières destinées aux transactions portant sur le transfert d'actifs incorporels ou de droits sur des actifs incorporels. La section D. 3 rassemble des instructions supplémentaires concernant le transfert d'actifs incorporels ou de droits sur des actifs incorporels dont la valeur est très incertaine au 
moment de la transaction. Enfin, la section D.4 présente des instructions supplémentaires relatives aux transactions portant sur l'utilisation d'actifs incorporels en lien avec la vente de biens ou services sans transfert de droits sur ces actifs.

\section{D.1. Principes généraux applicables aux transactions portant sur des actifs incorporels}

6.107 Les paragraphes 1.33-1.63 et le chapitre III présentent des principes à prendre en compte ainsi que le processus recommandé pour réaliser une analyse de comparabilité. Les prescriptions énoncées dans ces sections des Principes s'appliquent à toutes les transactions contrôlées portant sur des actifs incorporels.

6.108 La mise en application des prescriptions énoncées dans les Principes concernant le contenu d'une analyse de comparabilité et le processus correspondant dans le cas d'une transaction portant sur des actifs incorporels suppose la prise en compte, lors de l'analyse des prix de transfert, des options réalistes qui s'offrent à chacune des parties à la transaction. Les principes à suivre pour tenir compte de ces options réalistes sont exposés aux paragraphes 9.59-9.64.

6.109 La prise en compte des options réalistes qui s'offrent aux parties à la transaction conduit à examiner le point de vue de chaque partie à la transaction. Une analyse de comparabilité centrée uniquement sur l'une des parties à la transaction ne fournit pas, en règle générale, les éléments requis aux fins de l'évaluation d'une transaction portant sur des actifs incorporels (y compris lorsqu'une méthode unilatérale de fixation de prix de transfert est finalement retenue).

6.110 S'il importe de tenir compte, lors de l'analyse de comparabilité, $\mathrm{du}$ point de vue des deux parties à la transaction, les circonstances économiques propres à l'une des parties ne devraient pas être utilisées pour imposer une conclusion contraire aux options réalistes offertes à l'autre partie. Par exemple, on ne devrait pas attendre d'un cédant qu'il accepte de vendre tout ou partie de ses droits sur un actif incorporel à un prix moins avantageux pour lui que ses autres options réalistes (y compris la non-cession de ces droits), simplement parce que l'entreprise associée cessionnaire manque des ressources requises pour l'exploitation efficace des droits ainsi cédés. De même, on ne devrait pas attendre d'un cessionnaire qu'il accepte d'acquérir des droits sur un ou plusieurs actifs incorporels à un prix auquel il serait impossible pour lui d'envisager une exploitation rentable par son entreprise des droits ainsi acquis. Cette situation serait en 
effet moins favorable pour le cessionnaire que l'option réaliste qui s'offre à lui de renoncer à acquérir les droits.

6.111 Il sera souvent possible d'identifier, pour une transaction portant sur des actifs incorporels, un prix conforme aux options réalistes offertes à chacune des parties. L'existence d'un tel prix répond au principe selon lequel les groupes d'entreprises multinationales cherchent à optimiser l'allocation de leurs ressources. Lorsque le prix minimum acceptable par le cédant, compte tenu des options réalistes qui lui sont offertes, est supérieur au prix maximum acceptable par le cessionnaire, compte tenu des options réalistes dont ce dernier dispose, il peut être nécessaire d'examiner s'il convient soit de renoncer à la transaction au titre du deuxième cas mentionné au paragraphe 1.65, soit d'appliquer les principes énoncés aux paragraphes 9.34-9.38 ou au paragraphe 9.122, soit de modifier autrement les modalités de la transaction. De même, lorsqu'il est avancé que l'utilisation actuelle d'un actif incorporel ou qu'une option réaliste envisagée (telle qu'une autre utilisation de l'actif concerné) ne permet pas d'optimiser l'allocation des ressources, il pourrait être nécessaire d'examiner si ces affirmations sont compatibles avec les circonstances réelles du cas d'espèce. Ces discussions soulignent l'importance de prendre en compte l'ensemble des faits et circonstances entourant les transactions en vue de déterminer si celles-ci sont comparables.

\section{D.2. Instructions supplémentaires relatives aux transferts d'actifs incorporels ou de droits sur des actifs incorporels}

6.112 Cette section fournit des instructions supplémentaires en réponse aux difficultés propres aux transferts d'actifs incorporels ou de droits sur des actifs incorporels entre entreprises associées. Ces transactions recouvrent notamment les ventes d'actifs incorporels ainsi que les transactions économiquement équivalentes à une vente. Ces transactions peuvent aussi porter sur la concession de droits sur un ou plusieurs actifs incorporels ou sur une opération similaire. La finalité de cette section n'est pas de fournir des instructions exhaustives en matière de fixation des prix de transfert pour les actifs incorporels. Cette section complète les dispositions applicables par ailleurs énoncées aux chapitres I à III et les instructions figurant dans les sections A, B, C et D.1 du présent chapitre, dans le cas de transferts d'actifs incorporels ou de droits sur des actifs incorporels, en exposant des instructions adaptées aux difficultés particulières fréquemment soulevées par ces transferts. 
(i) Comparabilité des actifs incorporels ou des droits sur les actifs incorporels

6.113 Lors de la mise en application des dispositions des chapitres I à III dans le cas d'une transaction portant sur le transfert d'actifs incorporels ou de droits sur des actifs incorporels, il convient de reconnaître que de nombreux actifs incorporels présentent des caractéristiques spécifiques en vertu desquelles les revenus et bénéfices futurs pourront différer grandement. Il est donc essentiel de tenir compte des caractéristiques spécifiques des actifs incorporels lors de la réalisation d'une analyse de comparabilité portant sur un transfert de tels actifs. Cela est particulièrement important si la méthode de prix de transfert retenue comme étant la plus appropriée est la méthode du prix comparable ou une autre méthode utilisant des comparables. Dans le cas du transfert d'un actif incorporel ou de droits sur un actif incorporel fournissant à l'entreprise un avantage compétitif unique sur le marché, les transactions ou actifs incorporels réputés comparables doivent être analysés attentivement. Il est essentiel de s'assurer que les comparables identifiés offrent dans les faits un potentiel de bénéfices similaire.

6.114 Les paragraphes suivants exposent certaines des caractéristiques propres aux actifs incorporels qui peuvent être pertinentes en vue d'une analyse de comparabilité portant sur des transferts d'actifs incorporels ou de droits sur de tels actifs. La liste proposée ci-après n'est pas exhaustive et, selon le cas d'espèce, la prise en compte de facteurs supplémentaires ou différents pourra s'avérer essentielle pour réaliser une analyse de comparabilité.

\section{Exclusivité}

6.115 La nature exclusive ou non exclusive des droits sur les actifs incorporels qui font l'objet d'une transaction portant sur le transfert d'actifs incorporels ou de droits sur des actifs incorporels peut être un élément de comparabilité important. Dans certains cas, le propriétaire légal d'un actif incorporel est en droit d'exclure des tiers de l'utilisation de cet actif. Par exemple, un brevet est assorti d'un droit exclusif, valable plusieurs années, d'utiliser l'invention brevetée. Si une partie qui contrôle des droits sur des actifs incorporels peut exclure d'autres entreprises du marché, ou leur interdire l'utilisation d'actifs incorporels qui procurent un avantage commercial, elle peut disposer d'un niveau élevé de pouvoir ou d'influence sur le marché. Une partie qui détient des droits non exclusifs sur des actifs incorporels n'est pas en mesure d'exclure tous les concurrents et ne dispose pas, en règle générale, d'un tel niveau de pouvoir ou d'influence sur le marché. Il importe donc de tenir compte, aux fins d'une analyse de 
comparabilité, de la nature exclusive ou non exclusive des droits sur les actifs incorporels.

\section{Portée et durée de la protection juridique}

6.116 La portée et durée de la protection juridique des actifs incorporels concernés par un transfert peuvent être des éléments de comparabilité importants. Les protections juridiques associées à certains actifs incorporels peuvent empêcher les concurrents d'une entreprise d'entrer sur un marché donné. Dans le cas d'autres catégories d'actifs incorporels, comme le savoir-faire ou les secrets industriels ou commerciaux, les protections juridiques peuvent être d'une nature différente et se révéler moins efficaces ou moins durables. Si les actifs incorporels ont une durée d'utilité réduite, la durée des protections juridiques peut être un élément pertinent, la durée des droits sur les actifs incorporels ayant une incidence sur les attentes des parties à la transaction en matière de bénéfices générés par l'exploitation des actifs concernés. Par exemple, deux brevets par ailleurs comparables n'ont pas la même valeur si l'un expire après douze mois tandis que l'autre demeure valable dix ans.

6.117 La couverture géographique des actifs incorporels ou des droits sur des actifs incorporels est un élément de comparabilité important. Selon la nature des produits, des actifs incorporels et des marchés concernés, la concession de droits mondiaux sur des actifs incorporels peut avoir une valeur supérieure à une concession de droits limités à un ou plusieurs pays.

\section{Durée d'utilité}

6.118 De nombreux actifs incorporels ont une durée d'utilité réduite. Comme indiqué plus haut, la durée d'utilité d'un actif incorporel peut dépendre de la nature et de la durée des protections juridiques qui lui sont associées. Parmi les autres facteurs qui influencent la durée d'utilité de certains actifs incorporels figurent le rythme des évolutions technologiques dans le secteur concerné et l'apparition de produits nouveaux et potentiellement améliorés. Dans certains cas, la durée d'utilité d'un actif incorporel peut être prolongée.

6.119 Lors de la réalisation d'une analyse de comparabilité, il conviendra donc de tenir compte de la durée d'utilité prévisionnelle des actifs incorporels concernés. En règle générale, et toutes choses étant égales par ailleurs, les actifs incorporels devant procurer des avantages commerciaux sur une période plus longue se verront attribuer une valeur 
plus élevée que d'autres actifs incorporels similaires mais procurant ces avantages sur une période plus courte. En outre, l'évaluation de la durée d'utilité d'un actif incorporel doit également tenir compte de l'utilisation qui est faite de cet actif. La durée d'utilité d'un actif incorporel qui sous-tend des activités continues de recherche- développement peut ainsi s'étendre au-delà de la vie commerciale de la première génération de produits liés à cet actif incorporel.

\section{Avancement du développement}

6.120 L'avancement du développement de certains actifs incorporels peut être un critère pertinent aux fins d'une analyse de comparabilité. Il est fréquent qu'un actif incorporel soit transféré dans le cadre d'une transaction contrôlée avant même que la viabilité commerciale des produits qui lui sont liés ait été démontrée. L'industrie pharmaceutique est un exemple souvent cité, puisqu'un composé chimique peut être breveté et les brevets correspondants (ou les droits d'utilisation de ces brevets) peuvent être transférés dans le cadre d'une transaction contrôlée bien avant que les activités de recherche-développement et les essais aient démontré que ce composé chimique permet un traitement sûr et efficace d'une pathologie donnée.

6.121 En règle générale, les actifs incorporels liés à des produits dont la viabilité commerciale est établie se verront attribuer une valeur plus élevée que d'autres actifs incorporels par ailleurs comparables mais liés à des produits dont la viabilité commerciale reste à démontrer. Lors d'une analyse de comparabilité portant sur des actifs incorporels en cours de mise au point, il convient d'évaluer la probabilité que les phases ultérieures de leur mise au point permettent de générer des retombées commerciales importantes dans le futur. Dans certains cas, il est utile de consulter les données sectorielles concernant les risques associés aux phases ultérieures de mise au point des actifs. En tout état de cause, les circonstances propres au cas d'espèce doivent être prises en compte.

Droits relatifs aux améliorations, révisions et mises à jour

6.122 Les droits des parties sur les améliorations, révisions et mises à jour relatives aux actifs incorporels constituent souvent un élément important aux fins d'une analyse de comparabilité portant sur ces actifs. Les produits protégés par des actifs incorporels peuvent, dans certains secteurs, devenir relativement vite obsolètes ou non compétitifs en l'absence d'efforts continus de mise au point et d'amélioration des actifs incorporels concernés. Par conséquent, un accès aux mises à jour et aux améliorations peut permettre de transformer des avantages de court terme tirés des actifs 
incorporels en avantages sur un plus long terme. En vue d'une analyse de comparabilité, il importe donc de préciser si une concession de droits sur des actifs incorporels inclut ou non un accès aux améliorations, révisions et mises à jour relatives aux actifs concernés.

6.123 Une question très similaire, souvent importante en vue d'une analyse de comparabilité, consiste à savoir si le cessionnaire d'actifs incorporels obtient le droit d'utiliser ces actifs dans le cadre d'activités de recherche destinées à mettre au point des actifs nouveaux et améliorés. Par exemple, le droit d'utiliser une plate-forme logicielle existante comme point de départ pour développer de nouveaux produits logiciels peut raccourcir les délais de mise au point et permettre à une entreprise d'être la première à commercialiser un nouveau produit ou une nouvelle application, plutôt que de rejoindre un marché déjà occupé par des produits concurrents. Il importe donc, en vue d'une analyse de comparabilité portant sur des actifs incorporels, de tenir compte des droits des parties à utiliser ces actifs pour mettre au point des versions nouvelles et améliorées de leurs produits.

\section{Avantages escomptés}

6.124 Les différentes questions de comparabilité examinées ci-après influent sur les anticipations des parties à la transaction quant aux avantages que procurera l'utilisation des actifs incorporels considérés. Lorsque les avantages escomptés de l'utilisation de deux actifs incorporels diffèrent largement, il est difficile de considérer ces deux actifs comme suffisamment comparables aux fins d'une analyse de prix de transfert à défaut d'ajustements de comparabilité fiables. En particulier, la rentabilité réelle et potentielle des produits, existants ou à venir, qui sont liés à un actif incorporel doit entrer en ligne de compte. Il est peu probable que des actifs incorporels liés à des produits ou services offrant une rentabilité élevée puissent être comparés à d'autres actifs incorporels liés à des produits ou services dont la rentabilité est simplement égale à la moyenne du secteur. Tous les facteurs ayant une influence sensible sur les avantages que les parties à une transaction contrôlée pensent retirer d'un actif incorporel doivent être pris en compte lors de la réalisation d'une analyse de comparabilité.

\section{(ii) Comparaison des risques dans les cas de transferts d'actifs incorporels ou de droits sur des actifs incorporels}

6.125 En vue d'une analyse de comparabilité portant sur le transfert d'actifs incorporels ou de droits sur des actifs incorporels, il convient de tenir compte des risques liés à la probabilité d'obtenir des avantages économiques issus des actifs incorporels, et d'analyser la répartition des 
risques entre les parties selon le cadre défini à la section B.2. de la partie I $\mathrm{du}$ chapitre IX. Les catégories de risques décrites ci-après devraient, notamment, entrer en ligne de compte pour décider si des transferts d'actifs incorporels ou de combinaisons d'actifs incorporels sont des transactions comparables, et si des actifs incorporels eux-mêmes sont comparables.

- Risques liés à l'évolution future des actifs incorporels. Les questions soulevées ici sont les suivantes: les actifs incorporels sont-ils à ce jour liés à des produits commercialement viables et pourraient-ils l'être à l'avenir; quel est le coût prévisionnel des futures activités de mise au point et de tests; quelle est la probabilité que ces activités soient couronnées de succès; et d'autres questions similaires. Il importe particulièrement de tenir compte des risques propres à la phase de mise au point lorsque les transactions portent sur le transfert d'actifs incorporels en cours de mise au point.

- Risques liés à l'obsolescence des produits et à la perte de valeur des actifs incorporels. Cette catégorie de risques renvoie à la probabilité que des concurrents lancent à l'avenir des produits et des services pouvant réduire de manière significative le marché des produits liés aux actifs incorporels soumis à l'analyse.

- Risques liés aux violations de droits sur des actifs incorporels. Cette catégorie de risques suppose d'évaluer plusieurs éléments : la probabilité que des tiers obtiennent gain de cause après avoir fait valoir que des produits liés à des actifs incorporels portent atteinte à leurs propres droits sur des actifs incorporels ; le montant des frais probablement supportés pour se défendre en cas de poursuites de cette nature ; la probabilité que le titulaire de droits sur des actifs incorporels parvienne effectivement à empêcher des tiers de porter atteinte à ses droits; le risque que des produits de contrefaçon n'abaissent la rentabilité des marchés visés; et la probabilité que des dommages-intérêts importants soient accordés en cas de violation des droits.

- Responsabilité au titre des produits et risques similaires liés à l'utilisation future des actifs incorporels.

(iii) Ajustements de comparabilité concernant des transferts d'actifs incorporels ou de droits sur des actifs incorporels

6.126 Les prescriptions énoncées aux paragraphes 3.47-3.54 relatives aux ajustements de comparabilité s'appliquent aux transactions portant sur le transfert d'actifs incorporels ou de droits sur des actifs incorporels. Il 
importe de noter que les différences entre actifs incorporels peuvent avoir des conséquences économiques significatives et qu'il peut être difficile de réaliser de manière fiable l'ajustement requis. En particulier, lorsque les montants associés aux ajustements de comparabilité représentent un pourcentage élevé du prix de l'actif incorporel transféré, il peut être fondé de penser, selon les circonstances particulières du cas d'espèce, que le calcul de l'ajustement n'est pas fiable et que les actifs incorporels retenus ne sont en fait pas suffisamment comparables pour être pris en compte aux fins d'une analyse de prix de transfert valable. Si aucun ajustement de comparabilité fiable n'est possible, il peut être nécessaire de choisir une méthode de prix de transfert moins dépendante de l'identification d'actifs incorporels comparables ou de transactions comparables.

\section{(iv) Utilisation de comparables issus de bases de données}

6.127 La comparabilité et la possibilité de réaliser des ajustements de comparabilité revêtent une importance particulière dans l'examen des actifs incorporels potentiellement comparables et des taux de redevance tirés de bases de données commerciales ou de recueils spécifiques de contrats de licence ou d'accords similaires accessibles au public. Les principes énoncés aux paragraphes 3.30-3.34 s'appliquent pleinement pour évaluer l'intérêt des transactions répertoriées dans ces sources. Il importe notamment d'évaluer si les informations publiques issues de bases de données du commerce et de recueils spécifiques sont suffisamment détaillées pour permettre d'évaluer les caractéristiques particulières des actifs incorporels qui peuvent s'avérer importantes dans la réalisation d'une analyse de comparabilité. Lors de l'examen de contrats de licence comparables issus de bases de données, les circonstances propres au cas d'espèce, y compris la méthodologie appliquée, doivent entrer en ligne de compte à la lumière des dispositions du paragraphe 3.38 .

Sélection de la méthode de prix de transfert la plus appropriée aux transactions portant sur un transfert d'actifs incorporels ou de droits sur des actifs incorporels

6.128 Les prescriptions prévues par les présents Principes concernant le choix de la méthode de prix de transfert la plus appropriée aux circonstances du cas d'espèce sont énoncées aux paragraphes 2.1-2.12. Ces principes s'appliquent pleinement aux cas portant sur le transfert d'actifs incorporels ou de droits sur des actifs incorporels. Lors de la sélection de la méthode de prix de transfert la plus appropriée aux transactions portant sur un transfert d'actifs incorporels ou de droits sur des actifs incorporels, il convient d'accorder une attention particulière aux éléments suivants : (i) la nature des actifs incorporels concernés; (ii) les difficultés rencontrées fréquemment, 
voire le plus souvent, pour identifier des transactions sur le marché libre et des actifs incorporels potentiellement comparables; et (iii) la difficulté à appliquer certaines méthodes de prix de transfert décrites au chapitre II à des transactions portant sur un transfert d'actifs incorporels. Les questions examinées ci-après revêtent une importance particulière dans la sélection des méthodes de prix de transfert conformément aux Principes.

\subsection{Lors de l'application des principes énoncés aux} paragraphes 2.1-2.12 à des transactions portant sur le transfert d'actifs incorporels ou de droits sur des actifs incorporels, il importe de souligner que des transactions structurées de manière différente peuvent avoir des conséquences économiques similaires. Par exemple, la fourniture d'un service utilisant des actifs incorporels peut avoir des conséquences économiques très similaires à celles d'une transaction portant sur un transfert d'actif incorporel (ou de droits sur un actif incorporel) puisque, dans les deux cas, la valeur de l'actif incorporel peut être transmise au cessionnaire. En conséquence, lors de la sélection de la méthode de prix de transfert la plus appropriée à une transaction portant sur un transfert d'actifs incorporels ou de droits sur des actifs incorporels, il importe de prendre en compte les conséquences économiques de la transaction, plutôt que de suivre une classification arbitraire.

6.130 Ce chapitre établit clairement que, dans le cas de transactions portant sur le transfert d'actifs incorporels ou de droits sur des actifs incorporels, il est important de ne pas considérer simplement que la totalité $\mathrm{du}$ bénéfice résiduel, obtenu après déduction de revenus limités correspondant à la rémunération des fonctions exercées, devrait nécessairement être attribuée au propriétaire des actifs incorporels. La sélection de la méthode de prix de transfert la plus appropriée devrait s'appuyer sur une analyse fonctionnelle permettant une compréhension claire des processus opérationnels à l'échelle mondiale de l'entreprise multinationale concernée et de la manière dont les actifs incorporels transférés interagissent avec d'autres fonctions, actifs et risques constitutifs $\mathrm{du}$ fonctionnement à l'échelle mondiale de l'entreprise. L'analyse fonctionnelle devrait identifier les facteurs de création de valeur, qui peuvent notamment inclure les risques encourus, les caractéristiques du marché, le lieu considéré, les stratégies d'entreprise ou les synergies obtenues au sein d'un groupe d'entreprises multinationales. Tous les facteurs pertinents ayant une contribution significative à la création de valeur devraient entrer en ligne de compte lors de la sélection de la méthode de prix de transfert et lors de tout ajustement apporté à cette méthode sur le fondement de l'analyse de comparabilité. 
6.131 Les principes énoncés aux paragraphes 2.12, 3.58 et 3.59 concernant l'utilisation de plusieurs méthodes de prix de transfert sont applicables aux transactions portant sur le transfert d'actifs incorporels ou de droits sur des actifs incorporels.

6.132 Les paragraphes 3.9-3.12 et le paragraphe 3.37 fournissent des instructions relatives à l'agrégation de transactions aux fins d'une analyse de prix de transfert. Ces principes s'appliquent pleinement aux transactions portant sur le transfert d'actifs incorporels ou de droits sur des actifs incorporels et sont complétés par les instructions énoncées à la section $\mathrm{C}$ du présent chapitre VI. De fait, les transferts d'actifs incorporels concernent souvent un ensemble d'actifs incorporels, ou sont souvent associés à des transactions portant sur une vente de biens ou sur une prestation de services. Dans ce cas, l'analyse de prix de transfert la plus pertinente est probablement celle qui tient compte des relations étroites entre les différentes transactions agrégées de manière à améliorer la fiabilité de l'analyse.

(vi) Instructions supplémentaires sur les méthodes de prix de transfert dans le cas de transactions portant sur le transfert d'actifs incorporels ou de droits sur des actifs incorporels.

6.133 Selon les circonstances spécifiques du cas d'espèce, chacune des cinq méthodes de prix de transfert de l'OCDE décrites au chapitre II peut s'avérer être la plus appropriée lorsque la transaction porte sur le transfert contrôlé d'un ou de plusieurs actifs incorporels. Le recours à des méthodes alternatives peut également être approprié.

6.134 Lorsque l'analyse de comparabilité identifie des informations fiables concernant des transactions comparables sur le marché libre, la fixation de prix de pleine concurrence pour le transfert d'actifs incorporels ou de droits sur des actifs incorporels peut s'appuyer sur ces comparables, après avoir apporté les ajustements appropriés et fiables.

6.135 Toutefois, l'analyse de comparabilité (notamment l'analyse fonctionnelle) relative à des transferts d'actifs incorporels ou de droits sur des actifs incorporels révèlera souvent l'absence de transactions comparables sur le marché libre permettant d'établir le prix de pleine concurrence et les autres conditions d'une transaction. C'est le cas en particulier lorsque les actifs incorporels concernés présentent des caractéristiques uniques, ou s'ils revêtent une importance telle qu'ils ne sont transférés qu'entre entreprises associées. D'autres facteurs peuvent conduire à la même conclusion, en particulier l'absence de données disponibles 
concernant des transactions potentiellement comparables. Malgré les difficultés liées au manque de comparables fiables, il est généralement possible de fixer le prix de pleine concurrence et les autres conditions d'une transaction contrôlée, hormis lorsque les dispositions du paragraphe 1.65 s'appliquent.

6.136 Lorsqu'il est impossible d'identifier des informations fiables concernant des transactions comparables sur le marché libre, le principe de pleine concurrence conduit à appliquer une autre méthode de calcul du prix dont des tiers indépendants auraient convenu dans des circonstances comparables. En vue de ce calcul, les éléments suivants doivent entrer en ligne de compte :

- $\quad$ les fonctions exercées, les actifs détenus et les risques assumés par les parties à la transaction ;

- les motivations opérationnelles qui ont motivé la réalisation de la transaction ;

- les perspectives de chaque partie à la transaction et les options réalistes qui s'offrent à elle ;

- les avantages concurrentiels conférés par les actifs incorporels, notamment la rentabilité relative des produits et services, existants ou potentiels, liés aux actifs incorporels ;

- les avantages économiques escomptés de la réalisation de la transaction ;

- d'autres facteurs de comparabilité, tels que les caractéristiques des marchés locaux, les économies de localisation, le collectif de travail et les synergies obtenues au sein d'un groupe d'entreprises multinationales.

6.137 Lors de l'identification des prix et des autres conditions dont auraient convenu des tiers indépendants dans des circonstances comparables, il importe souvent d'identifier avec soin les aspects caractéristiques d'une transaction contrôlée qui découlent de la relation entre les parties. Les entreprises associées ne sont pas tenues de structurer leurs transactions précisément selon les modalités que des tiers indépendants auraient retenues. Toutefois, lorsque les modalités adoptées par des entreprises associées pour structurer leurs transactions diffèrent de celles habituellement retenues par des tiers indépendants, il convient de tenir compte des effets de ces structures sur les prix et les autres conditions des transactions dont des tiers indépendants auraient convenu dans des 
circonstances comparables, afin d'évaluer les avantages potentiellement retirés par chacune des parties dans des conditions de pleine concurrence.

6.138 Une attention particulière est de mise dans l'application de certaines méthodes de prix de transfert de l'OCDE à des transactions portant sur le transfert d'actifs incorporels ou de droits sur des actifs incorporels. Les méthodes unilatérales, y compris la méthode du prix de revente et la méthode transactionnelle de la marge nette, ne sont pas, en règle générale, des méthodes fiables aux fins de la valorisation directe d'actifs incorporels. Ces approches peuvent quelquefois être utilisées pour valoriser indirectement des actifs incorporels, en les utilisant pour attribuer à certaines fonctions des valeurs à partir desquelles est déduite la valeur résiduelle des actifs incorporels. Cependant, il est alors important de garder à l'esprit les principes exposés au paragraphe 6.130 et de veiller à ce que la totalité des fonctions, risques, actifs et autres facteurs contribuant à la génération de revenus soit correctement identifiée et évaluée.

6.139 L'utilisation de méthodes de prix de transfert cherchant à estimer la valeur d'un actif incorporel à partir du coût de mise au point de cet actif est généralement déconseillée. En effet, le coût de mise au point d'un actif incorporel est rarement corrélé avec sa valeur et son prix de transfert ultérieurs. En conséquence, il convient habituellement de renoncer aux méthodes de fixation des prix de transfert qui reposent sur le coût de mise au point d'un actif incorporel.

6.140 Toutefois, dans certains cas limités, les méthodes de prix de transfert fondées sur le coût estimatif de reproduction ou de remplacement de l'actif incorporel peuvent être employées. Ces approches peuvent s'avérer valables dans le cas de la mise au point d'actifs incorporels utilisés au titre d'activités internes (par exemple, des logiciels internes), en particulier lorsque les actifs incorporels concernés ne sont pas des actifs incorporels uniques et de valeur. Lorsque les actifs incorporels concernés sont liés à des produits déjà commercialisés, cependant, les méthodes fondées sur le coût de remplacement soulèvent d'importants problèmes de comparabilité. Il importe alors, entres autres questions à trancher, d'évaluer les effets sur la valeur des actifs incorporels de tout report du processus de mise au point. En effet, le premier entrant sur un marché retire souvent des avantages significatifs du lancement précoce d'un produit. En conséquence, la valeur d'un produit identique (et des actifs incorporels liés) développé ultérieurement sera inférieure à celle d'un produit de référence (et des actifs incorporels liés) déjà présent sur un marché. Dans ce cas, le coût de remplacement estimé ne fournira pas d'évaluation satisfaisante de la valeur d'un actif incorporel objet d'un transfert. De même, lorsqu'un actif 
incorporel fait l'objet de protections juridiques ou présente des caractéristiques d'exclusivité, la valeur découlant du droit d'exclure des concurrents de l'utilisation de l'actif incorporel ne sera pas reflétée par une analyse fondée sur le coût de remplacement. Les évaluations reposant sur les coûts ne sont généralement pas fiables lorsqu'elles sont utilisées pour déterminer le prix de pleine concurrence d'actifs incorporels en cours de développement.

6.141 Les dispositions du paragraphe 2.10 relatives à l'utilisation d'approches empiriques s'appliquent à la détermination d'un prix de transfert approprié pour toute transaction contrôlée, y compris en cas d'utilisation ou de transfert d'actifs incorporels. En conséquence, une méthode empirique ne peut pas être utilisée pour établir si un prix ou une répartition des revenus respecte bien les conditions de pleine concurrence, notamment dans le cas d'une répartition des revenus entre un cédant d'actifs incorporels et son cessionnaire.

6.142 Les méthodes de prix de transfert présentant la plus forte probabilité d'être utiles dans les situations de transferts d'un ou de plusieurs actifs incorporels sont la méthode du prix comparable sur le marché libre et la méthode transactionnelle du partage des bénéfices. Des techniques d'évaluation peuvent également s'avérer utiles. Des instructions supplémentaires concernant les méthodes de prix de transfert ayant les plus fortes probabilités d'être utiles dans le cas de transferts d'actifs incorporels sont présentées ci-après.

(a) Application de la méthode du prix comparable sur le marché libre

6.143 Lorsque des transactions comparables sur le marché libre ont été identifiées de manière fiable, la méthode du prix comparable sur le marché libre peut être appliquée pour déterminer les conditions de pleine concurrence d'un transfert d'actifs incorporels ou de droits sur des actifs incorporels. Les principes généraux figurant dans les paragraphes 2.14-2.21 s'appliquent lorsque la méthode du prix comparable sur le marché libre est utilisée en lien avec des transactions portant sur le transfert d'actifs incorporels. Lors de l'utilisation de la méthode du prix comparable sur le marché libre en lien avec le transfert d'actifs incorporels, il importe notamment de veiller à la comparabilité entre les actifs incorporels ou droits sur des actifs incorporels qui font l'objet de la transaction contrôlée et ceux faisant l'objet de transactions sur le marché libre potentiellement comparables. Les facteurs de comparabilité présentés aux paragraphes 1.38-1.63 doivent entrer en ligne de compte. Les éléments 
exposés dans les sections D.2. (i) à (iv) du présent chapitre revêtent une importance particulière pour l'évaluation de la comparabilité d'actifs incorporels particuliers faisant l'objet d'un transfert et pour la réalisation des ajustements de comparabilité, lorsque c'est possible. Il convient de garder à l'esprit que, bien souvent, lorsque des actifs incorporels sont concernés, l'identification de comparables fiables peut s'avérer difficile, voire impossible.

6.144 Dans certains cas, des actifs incorporels achetés par un groupe d'entreprises multinationales auprès d'entreprises indépendantes sont transférés, immédiatement après l'achat, à un membre du groupe en question au moyen d'une transaction contrôlée. Le prix payé pour les actifs incorporels fournira souvent (après les ajustements appropriés, notamment au titre des actifs acquis ne faisant pas l'objet d'un nouveau transfert) un comparable utile pour déterminer le prix de pleine concurrence de la transaction contrôlée selon la méthode du prix comparable sur le marché libre. Selon les faits et circonstances du cas d'espèce, le prix d'achat auprès du tiers pourra alors être utile pour déterminer le prix de pleine concurrence et les autres conditions de la transaction contrôlée, même si les actifs incorporels sont acquis indirectement au travers d'un achat de titres de participation, ou si le prix versé au tiers concerné pour acquérir des titres de participation ou des actifs dépasse la valeur comptable des actifs ainsi achetés. Les exemples 24 et 27 de l'annexe au chapitre VI illustrent le contenu du présent paragraphe.

\section{[(b) Application des méthodes de partage des bénéfices}

6.145 Dans certains cas, une méthode transactionnelle du partage des bénéfices peut être utilisée pour déterminer les conditions de pleine concurrence d'un transfert d'actifs incorporels ou de droits sur des actifs incorporels lorsqu'il est impossible d'identifier des transactions comparables sur le marché libre. Les paragraphes 2.109-2.146 contiennent des instructions devant être prises en compte lors de l'utilisation de méthodes de partage des bénéfices. Ces instructions sont pleinement applicables aux situations portant sur le transfert d'actifs incorporels ou de droits sur des actifs incorporels. Lors de l'évaluation de la fiabilité des méthodes de partage des bénéfices, il convient de tenir compte de la disponibilité de données fiables et appropriées relatives aux bénéfices combinés et à la répartition correcte des dépenses, d'une part, et de la fiabilité des paramètres utilisés pour répartir les bénéfices combinés, d'autre part. Voir paragraphe 2.115 .

6.146 Les méthodes de partage des bénéfices peuvent être appliquées en lien avec la vente de la totalité des droits sur des actifs incorporels. 
Comme dans les autres cas d'application de méthodes de partage des bénéfices, une analyse fonctionnelle complète, tenant compte des fonctions exercées, des actifs détenus et des risques assumés par chacune des parties est un élément essentiel de l'analyse. Lorsqu'une analyse du partage des bénéfices est fondée sur les dépenses et revenus prévisionnels, il importe de tenir compte des préoccupations relatives à l'exactitude de ces prévisions mentionnées aux paragraphes 6.160-6.165.

6.147 Il est également parfois suggéré qu'une analyse de partage des bénéfices peut être appliquée aux transferts d'actifs incorporels en cours de mise au point. Aux fins d'une telle analyse, la valeur relative des contributions à la mise au point des actifs incorporels avant et après leur transfert peut être étudiée. Cela peut supposer que l'on cherche à amortir la contribution du cédant au titre de l'actif incorporel en cours de mise au point sur la durée d'utilité présumée de cette contribution, en faisant l'hypothèse d'une absence d'activités de mise au point ultérieures. Ces approches s'appuient en règle générale sur des estimations de flux de trésorerie et de bénéfices attendus après le transfert, et une fois menées à bien la phase de développement de l'actif incorporel.

6.148 Dans le cas d'actifs incorporels en cours de mise au point, la prudence est de mise lorsque des approches de partage des bénéfices sont utilisées soit pour évaluer les contributions des parties à la création des revenus au cours des années suivant le transfert, soit pour procéder à l'allocation des revenus futurs selon le principe de pleine concurrence. La contribution ou la valeur de travaux menés avant le transfert ne sont pas nécessairement corrélées au coût de ces travaux. Par exemple, un composé chimique pouvant déboucher sur une forme pharmaceutique à très fort potentiel commercial peut être mis au point dans un laboratoire pour un coût relativement modeste. De plus, une telle analyse de partage des bénéfices devrait prendre en compte toute une série de facteurs difficiles à évaluer. Il s'agit notamment des niveaux de risque relatifs et de la valeur des contributions des activités de recherche avant et après le transfert; du risque relatif et de ses effets sur la valeur pour les autres activités de mise au point menées avant et après le transfert ; du taux d'amortissement approprié pour différentes contributions à la valeur de l'actif incorporel; d'hypothèses concernant le calendrier de lancement de nouveaux produits potentiels ; ou de la valeur des contributions autres que celles provenant d'actifs incorporels à la génération effective des bénéfices. Dans ce contexte, les estimations des bénéfices et des flux de trésorerie peuvent s'avérer particulièrement incertaines. L'ensemble de ces facteurs peut conduire à s'interroger quant à la fiabilité d'une telle analyse de partage des bénéfices ${ }^{4}$. 
6.149 Lorsque des droits partiels sur des actifs incorporels dont la phase de mise au point est achevée sont transférés sous la forme d'une licence ou d'une transaction similaire et qu'il est impossible d'identifier des transactions comparables sur le marché libre, une approche de partage des bénéfices peut souvent être utilisée pour évaluer les contributions respectives des parties à l'obtention des bénéfices combinés. La contribution aux bénéfices émanant des droits sur les actifs incorporels mis à disposition par le donneur de licence ou le cédant des droits serait, dans ce cas, l'un des facteurs contribuant à l'obtention des bénéfices après le transfert. Toutefois, d'autres facteurs devraient également être pris en compte, tout particulièrement les fonctions exercées et les risques assumés par le preneur de licence/cessionnaire. Parmi les autres facteurs pertinents entrant en ligne de compte, citons les autres actifs incorporels utilisés par le donneur de licence/cédant et par le preneur de licence/cessionnaire dans leurs activités respectives. Lors d'une telle analyse, il importe d'examiner avec soin les limites définies dans les conditions générales du transfert quant à l'utilisation des actifs incorporels par le preneur de licence/cessionnaire et quant aux droits du donneur de licence/cédant à utiliser ces actifs dans la poursuite de ses activités de recherche-développement. En outre, il peut être opportun d'évaluer les contributions du preneur de licence à l'augmentation de la valeur des actifs incorporels qui font l'objet de la licence. Dans cette approche, l'allocation des bénéfices dépendra des conclusions de l'analyse fonctionnelle, laquelle inclura une analyse des principaux risques encourus. Il ne faut pas présumer qu'une analyse de partage des bénéfices relative à un accord de licence doit conduire à allouer tous les bénéfices résiduels, après déduction des revenus correspondant à la rémunération des fonctions exercées, au donneur de licence/cédant.]

\section{Utilisation des techniques d'évaluation}

6.150 Lorsque des transactions sur le marché libre comparables et fiables ne peuvent être identifiées pour le transfert d'un ou de plusieurs actifs incorporels, il est possible d'employer des techniques d'évaluation pour estimer le prix de pleine concurrence d'actifs incorporels transférés entre entreprises associées. En particulier, les techniques fondées sur l'évaluation des revenus, et plus précisément sur la valeur actualisée des flux de revenus ou de trésorerie futurs susceptibles d'être tirés de l'exploitation de l'actif incorporel objet de l'évaluation, peuvent s'avérer très utiles lorsqu'elles sont correctement utilisées. En fonction des faits et circonstances du cas d'espèce, les techniques d'évaluation peuvent être employées soit dans le cadre de l'une des cinq méthodes de prix de transfert approuvées par l'OCDE exposées au chapitre II, soit comme une approche de travail utile pour déterminer un prix de pleine concurrence. 
6.151 L'utilisation de techniques d'évaluation aux fins d'une analyse de prix de transfert portant sur le transfert d'actifs incorporels ou de droits sur des actifs incorporels doit se conformer au principe de pleine concurrence et aux prescriptions exposées dans les présents Principes. Il importe notamment de tenir compte des prescriptions énoncées aux chapitres I à III. Les principes relatifs aux options réalistes (voir paragraphe 1.34), aux points de vue des parties, à l'allocation des risques (voir paragraphes 9.10-9.46), et à l'agrégation des transactions (voir paragraphes 3.9-3.12) s'appliquent pleinement à l'utilisation des techniques d'évaluation en vue d'une analyse de prix de transfert. Par ailleurs, les règles exposées dans les présents Principes concernant la sélection de la méthode de détermination des prix de transfert s'appliquent pour décider de l'opportunité de recourir à ces techniques d'évaluation (voir paragraphes 2.1-2.11). Les principes exposés dans les sections A, B, C et D.1 du présent chapitre IV s'appliquent également lorsqu'il est envisagé d'employer des techniques d'évaluation.

6.152 Il est essentiel d'examiner les hypothèses et autres arguments qui sous-tendent toute application des techniques d'évaluation. L'application des principes comptables reconnus peut conduire à des hypothèses prudentes en matière d'évaluation et d'estimation de la valeur des actifs figurant au bilan d'une entreprise. Cette prudence propre à l'approche comptable peut se traduire par des définitions trop restrictives en vue de la fixation des prix de transfert ou par des approches d'évaluation qui ne sont pas toujours conformes au principe de pleine concurrence. Lors de la fixation des prix de transfert, il ne convient donc pas de considérer que des évaluations réalisées à des fins comptables reflètent nécessairement des valorisations ou des prix de pleine concurrence à moins d'avoir examiné en détail les hypothèses sous-jacentes. En particulier, les évaluations d'actifs incorporels issues d'affectations de prix d'acquisition effectuées à des fins comptables ne sont pas concluantes aux fins de la fixation des prix de transfert et devraient être utilisées avec prudence, après examen attentif des hypothèses sous-jacentes.

6.153 Les présents Principes n'ont pas pour finalité de fournir une présentation exhaustive des techniques d'évaluation utilisées par les spécialistes. Ils ne visent pas non plus à valider ou rejeter tel ou tel recueil de normes d'évaluation utilisé par ces spécialistes ou par les comptables, ni à décrire en détail ou à valider une ou plusieurs techniques ou méthodes d'évaluation comme étant particulièrement adaptées en vue d'une analyse des prix de transfert. Cependant, lorsque les techniques d'évaluation sont appliquées en tenant dûment compte des présents Principes, des circonstances spécifiques du cas d'espèce, des pratiques et principes d'évaluation reconnus, et en veillant à la validité des hypothèses qui sous-tendent l'évaluation et à leur conformité avec le principe de pleine 
concurrence, ces techniques peuvent s'avérer utiles pour conduire une analyse des prix de transfert en l'absence de transactions comparables sur le marché libre. Il convient toutefois de se référer aux paragraphes 6.139 et 6.140 pour ce qui concerne la fiabilité des techniques d'évaluation fondées sur les coûts de mise au point des actifs incorporels et leur application.

6.154 Les techniques d'évaluation consistant à estimer la valeur actualisée des flux de trésorerie futurs susceptibles d'être tirés de l'exploitation des actifs incorporels transférés peuvent être particulièrement utiles lorsqu'elles sont correctement appliquées. Ces techniques présentent de nombreuses variantes. D'une manière générale, la valeur de l'actif incorporel est évaluée à partir de la valeur estimée des flux de trésorerie futurs que l'actif est susceptible de générer durant le reste de sa durée d'utilité prévisionnelle. Sa valeur est estimée en calculant la valeur actualisée des flux de trésorerie attendus ${ }^{5}$. Cette approche suppose, entre autres conditions, de disposer de données fiables et réalistes en matière de prévisions financières, de taux de croissance, de taux d'actualisation, de durée d'utilité des actifs incorporels et d'effets fiscaux de la transaction. Elle exige en outre, lorsque nécessaire, la prise en compte des valeurs finales. En fonction des faits et circonstances du cas d'espèce, la valeur actualisée des flux de trésorerie susceptibles d'être tirés de l'exploitation d'un actif incorporel devrait être calculée du point de vue de chacune des parties pour parvenir à un prix de pleine concurrence. Le prix de pleine concurrence s'établira à l'intérieur de la plage de valeurs actualisées estimées selon le point de vue du cédant et du cessionnaire. Les exemples 28 à 30 qui figurent dans l'annexe au chapitre VI illustrent le contenu du présent paragraphe.

(d) Préoccupations propres à l'application de méthodes fondées sur la valeur actualisée des flux de trésorerie prévisionnels

6.155 Lors de l'application des techniques d'évaluation, y compris celles fondées sur les flux de trésorerie prévisionnels, il convient de tenir compte de la volatilité potentielle des estimations de valeur obtenues. De légères modifications apportées à l'une des hypothèses qui sous-tendent le modèle d'évaluation ou à un ou plusieurs paramètres de l'évaluation peuvent conduire à de grandes variations de la valeur estimée de l'actif incorporel fournie par le modèle. Un faible changement dans le pourcentage du taux d'actualisation ou dans celui des taux de croissance retenus pour établir les prévisions financières, ou une petite modification apportée aux hypothèses relatives à la durée d'utilité de l'actif incorporel peuvent profondément altérer l'évaluation définitive. En outre, cette volatilité est souvent amplifiée 
lorsque des changements concernent simultanément deux hypothèses ou paramètres, voire davantage.

6.156 La fiabilité de l'estimation de la valeur d'un actif incorporel obtenue à partir d'un modèle d'évaluation dépend tout particulièrement de la fiabilité des hypothèses sous-jacentes et du fait que l'évaluateur ait fait preuve d'une diligence raisonnable et d'un jugement sûr dans la validation des hypothèses retenues et dans l'estimation des paramètres employés.

6.157 Le rôle déterminant des hypothèses sous-jacentes et des paramètres d'évaluation justifie que les contribuables et les administrations fiscales qui utilisent des techniques d'évaluation pour déterminer les prix de pleine concurrence d'actifs incorporels transférés doivent: décrire expressément chaque hypothèse utilisée dans la construction du modèle d'évaluation; énoncer les principes suivis lors du choix des paramètres d'évaluation; et se tenir prêts à soutenir le caractère raisonnable des hypothèses et paramètres d'évaluation considérés. De plus, à titre de bonne pratique, les contribuables qui utilisent des techniques d'évaluation devraient inclure dans leur documentation relative aux prix de transfert une analyse de sensibilité montrant les effets que des changements dans les hypothèses et les paramètres retenus auraient sur la valeur estimée des actifs incorporels concernés.

6.158 En vue d'estimer la fiabilité d'un modèle d'évaluation, il peut être approprié de connaître la finalité première de l'évaluation et d'examiner les hypothèses et paramètres d'évaluation retenus dans le cadre de différentes évaluations conduites par le contribuable à des fins autres que fiscales. Il serait souhaitable que l'administration fiscale concernée demande des explications concernant toute incohérence entre les hypothèses utilisées pour évaluer un actif incorporel en vue de déterminer des prix de transfert et celles utilisées à d'autres fins. Par exemple, une telle demande est appropriée lorsqu'une entreprise a utilisé des taux d'actualisation élevés lors d'une analyse de prix de transfert tandis qu'elle utilise habituellement des taux plus faibles pour évaluer des projets potentiels de fusions et d'acquisitions. Il en va de même lorsqu'il est affirmé que certains actifs incorporels ont une durée d'utilité réduite alors que, dans les prévisions réalisées dans d'autres contextes opérationnels, des actifs incorporels liés produisent des flux de trésorerie après la fin de la "durée d'utilité » annoncée en vue de la fixation des prix de transfert. Les évaluations utilisées par un groupe d'entreprises multinationales lorsque celui-ci prend ses décisions opérationnelles peuvent être plus fiables que celles menées aux seules fins d'une analyse des prix de transfert. 
6.159 Les sections ci-après identifient plusieurs caractéristiques devant entrer en ligne de compte dans l'évaluation de certaines hypothèses importantes qui sous-tendent les calculs d'un modèle d'évaluation fondé sur l'actualisation des flux de trésorerie. Elles permettent de déterminer si une technique d'évaluation donnée a été appliquée de manière fiable. Indépendamment des diverses préoccupations décrites ci-dessus et présentées en détail dans les paragraphes suivants, selon les circonstances, l'application d'une telle technique d'évaluation, que ce soit dans le cadre de l'une des cinq méthodes approuvées par l'OCDE ou comme une approche de travail utile, peut s'avérer plus fiable que n'importe quelle méthode de prix de transfert, en particulier en l'absence de transactions comparables sur le marché libre.

\section{(1) Degré d'exactitude des prévisions financières}

6.160 La fiabilité de l'évaluation d'un actif incorporel transféré obtenue par des techniques d'évaluation fondées sur l'actualisation des flux de trésorerie dépend de l'exactitude des prévisions de flux de trésorerie et de revenus futurs sur lesquelles repose cette évaluation. Toutefois, l'exactitude des prévisions financières étant subordonnée à des évolutions du marché à la fois inconnues et imprévisibles à la date de l'évaluation, ce qui explique la nature incertaine des prévisions, il est essentiel que les contribuables et les administrations fiscales examinent avec soin les hypothèses qui sous-tendent les prévisions relatives aux revenus et aux dépenses à venir.

6.161 Il peut être particulièrement important de connaître l'origine et la finalité des prévisions financières pour procéder à leur évaluation. Certains contribuables réalisent des prévisions financières de manière régulière au titre de la planification de leurs activités, notamment parce que la direction de l'entreprise concernée s'appuie sur ces prévisions pour prendre des décisions de nature commerciale ou en matière d'investissement. Il est fréquent que les prévisions établies par une entreprise dans une finalité de planification non fiscale soient plus fiables que celles réalisées aux seules fins fiscales ou aux seules fins d'une analyse de prix de transfert.

6.162 La durée couverte par les prévisions entre également en ligne de compte pour évaluer la fiabilité de celles-ci. Plus la période durant laquelle un actif incorporel est censé générer des flux de trésorerie positifs est étendue, moins est probable que les prévisions de revenus et de dépenses soient fiables.

6.163 Un autre élément de l'évaluation de la fiabilité des prévisions consiste à déterminer si le rendement des actifs incorporels et des produits et services qui leur sont liés a été clairement établi sur le plan financier. La 
prudence est toujours de mise avant de considérer que les résultats obtenus par le passé sont un indicateur fiable pour l'avenir, puisque de nombreux facteurs sont susceptibles de changer. Cependant, les résultats d'exploitation obtenus par le passé peuvent fournir des indications utiles sur le rendement à venir des produits et services liés aux actifs incorporels. Les prévisions relatives à des produits et services qui n'ont pas encore été commercialisés ou sont en cours de mise au point sont par nature moins fiables que celles qui s'appuient sur un historique de données.

6.164 La décision d'inclure ou non les coûts de mise au point dans les prévisions de flux de trésorerie doit tenir compte de la nature de l'actif incorporel transféré. Certains actifs incorporels peuvent avoir une durée d'utilité indéterminée et faire l'objet d'activités de mise au point continues. Dans ce cas, il est opportun d'inclure les coûts futurs de mise au point dans les prévisions de flux de trésorerie. Dans d'autres cas, comme dans celui des brevets, les prévisions portent sur un actif incorporel dont la phase de mise au point est terminée et qui ne constitue pas un socle pour la mise au point de nouveaux actifs incorporels. Dans cette situation, les coûts de mise au point ne devraient pas être inclus dans les prévisions de flux de trésorerie relatives à l'actif incorporel transféré.

6.165 Lorsque, pour les raisons mentionnées ci-dessus ou pour quelque autre motif, il est fondé de penser que les prévisions qui sous-tendent l'évaluation manquent de fiabilité ou sont incertaines, il importe de consulter les indications figurant à la section D.3 relatives aux situations dans lesquelles l'évaluation est très incertaine au moment de la transaction.

\section{(2)}

\section{Hypothèses relatives aux taux de croissance}

6.166 Le taux de croissance prévisionnel est un paramètre clé pour certaines prévisions de flux de trésorerie et doit à ce titre être examiné avec soin. Les prévisions de flux de trésorerie sont souvent fondées sur les flux de trésorerie existants (ou sur les premiers flux de trésorerie suivant le lancement du produit pour les actifs incorporels en cours de développement) multipliés par un pourcentage de taux de croissance. Dans ce cas, le mode de calcul du taux de croissance doit être étudié. En particulier, il est rare que les revenus tirés d'un produit donné augmentent selon un taux de croissance fixe sur une longue période. Il importe de faire preuve de prudence et de ne pas accepter trop rapidement des modèles simples utilisant des taux de croissance fixes alors que ce choix n'est justifié ni par une expérience acquise avec des produits et sur des marchés similaires, ni par des prévisions raisonnables sur la conjoncture commerciale à venir. En règle générale, une technique d'évaluation fondée sur des prévisions de flux de trésorerie futurs est mise en œuvre de manière fiable lorsqu'elle s'appuie sur une étude des 
tendances d'évolution des revenus et des dépenses réalisée à partir de l'expérience acquise dans le secteur et dans l'entreprise pour des produits similaires.

\section{Taux d'actualisation}

6.167 Le ou les taux d'actualisation employés pour calculer la valeur actualisée des flux de trésorerie prévisionnels sont un élément central d'un modèle d'évaluation. Le taux d'actualisation prend en compte la valeur temps de l'argent et les risques ou incertitudes relatifs aux flux de trésorerie prévisionnels. De légers changements apportés à certains taux d'actualisation pouvant déboucher sur de fortes variations de la valeur des actifs incorporels calculée grâce à ces techniques, il est essentiel que les contribuables et les administrations fiscales prêtent une grande attention aux analyses conduites et aux hypothèses retenues lors du choix du ou des taux d'actualisation employés dans le modèle d'évaluation.

6.168 Il n'existe pas d'approche unique pour établir un taux d'actualisation approprié dans tous les cas aux fins de la fixation des prix de transfert. Les contribuables et les administrations fiscales ne devraient pas considérer qu'un taux d'actualisation fondé sur l'approche du coût moyen pondéré du capital ou sur toute autre approche peut être utilisé dans toutes les analyses relatives aux prix de transfert pour lesquelles le calcul des taux d'actualisation appropriés est important. En effet, la détermination du taux d'actualisation approprié passe par un examen des circonstances, des risques et des flux de trésorerie propres à chaque cas d'espèce.

6.169 Lors de la fixation et de l'évaluation des taux d'actualisation, il importe de reconnaître que, dans certains cas, notamment si l'évaluation porte sur des actifs incorporels en cours de mise au point, les actifs incorporels figurent parmi les composantes les plus risquées de l'activité du contribuable. Il convient également d'avoir à l'esprit que certaines activités sont par nature plus risquées que d'autres et que certains flux de trésorerie présentent naturellement une volatilité plus forte que d'autres. Par exemple, la probabilité que les dépenses prévues pour la recherche-développement soient effectivement engagées peut être supérieure à celle de voir les prévisions de revenus effectivement atteintes. Les taux d'actualisation devraient donc refléter les niveaux de risque de l'ensemble des activités ainsi que la volatilité des différents flux de trésorerie prévus en tenant compte des circonstances propres à chaque cas d'espèce.

6.170 Certains risques pouvant être pris en compte tant lors de l'établissement des prévisions financières que lors de la fixation du taux 
d'actualisation, on veillera à ne pas comptabiliser ces risques à double titre lors des calculs d'actualisation.

\section{Durée d'utilité des actifs incorporels et valeurs finales}

6.171 Les techniques d'évaluation sont souvent fondées sur des prévisions des flux de trésorerie générés par l'exploitation de l'actif incorporel pendant sa durée d'utilité. Dans ce cas, la définition de la durée d'utilité réelle de l'actif incorporel est l'une des hypothèses clés qui sous-tendent le modèle d'évaluation.

6.172 La durée d'utilité prévisionnelle d'un actif incorporel doit être établie en examinant l'ensemble des faits et circonstances du cas d'espèce. La durée d'utilité d'un actif incorporel peut dépendre notamment de la nature et de la durée des protections juridiques dont il bénéficie. Elle peut également dépendre du rythme des évolutions technologiques et d'autres facteurs qui ont une incidence sur les conditions de concurrence dans l'environnement économique considéré. Voir à ce propos les paragraphes 6.118 et 6.119 .

6.173 Dans certains cas, des actifs incorporels peuvent continuer à alimenter les flux de trésorerie pendant plusieurs années après l'expiration des protections juridiques ou après le retrait du marché des produits qui leur étaient spécifiquement liés. Cela peut être notamment le cas lorsqu'une génération d'actifs incorporels constitue le socle de la mise au point des générations suivantes d'actifs incorporels et de nouveaux produits. Dans ce cas, il peut être justifié d'attribuer une fraction des flux de trésorerie générés par de nouveaux produits encore à l'état de projet à des actifs incorporels parvenus, quant à eux, au terme de leur durée d'utilité. Il convient par ailleurs de reconnaître que, si certains actifs incorporels ont une durée d'utilité indéterminée au moment de l'évaluation, cela ne signifie pas pour autant que des revenus exceptionnels leur seront indéfiniment attribuables.

6.174 À cet égard, lorsqu'un actif incorporel alimente des flux de trésorerie au-delà de la période couverte par les prévisions financières raisonnables, on pourra être amené à calculer la valeur finale des flux de trésorerie générés par cet actif. Si une évaluation utilise des valeurs finales, les hypothèses formulées pour leur calcul doivent être clairement énoncées et les hypothèses sous-jacentes examinées en détail, notamment pour ce qui concerne les taux de croissance. 
6.175 Lorsque la technique d'évaluation vise à isoler les flux de trésorerie prévisionnels générés par un actif incorporel, il peut être nécessaire d'évaluer et de quantifier les effets sur les prévisions de flux de trésorerie des futurs impôts sur les bénéfices prévisionnels. Les éléments fiscaux à prendre en compte couvrent notamment: (i) les impôts prévisionnels qui devraient grever les futurs flux de trésorerie; (ii) les réductions d'impôt liées à l'amortissement fiscal dont le cessionnaire pourrait, le cas échéant, bénéficier ; (iii) les impôts que le cédant devrait, le cas échéant, acquitter au titre du transfert.

\section{(vii) Conditions de paiement}

6.176 Les contribuables disposent d'une grande latitude concernant les conditions de paiement des actifs incorporels transférés. Lors d'une transaction entre parties indépendantes, ces paiements prennent souvent la forme d'un versement unique pour un montant forfaitaire. Il est également fréquent d'observer des versements périodiques pendant une durée déterminée. Les accords portant sur des versements périodiques peuvent donner lieu soit à une série de paiements partiels d'un montant égal, soit à des paiements conditionnels dont le montant varie en fonction du volume de vente des produits liés aux actifs incorporels, de la rentabilité, ou d'un autre paramètre. Les principes énoncés au chapitre I (paragraphes 1.48-1.69) devraient être suivis lors de l'analyse des accords conclus par un contribuable en matière de conditions de paiement.

6.177 Lors de l'examen des dispositions relatives aux conditions de paiement prévues par les accords conclus par un contribuable, il convient de souligner que certaines modalités de paiement supposent des risques plus ou moins importants pour l'une des parties. Par exemple, un paiement conditionné aux ventes ou bénéfices à venir suppose généralement un risque plus élevé pour le cédant que le versement unique d'un montant forfaitaire ou qu'une série de paiements partiels d'un montant égal, du fait de l'existence d'une condition. Les conditions de paiement retenues doivent être appropriées au regard des faits et circonstances du cas d'espèce, y compris les accords conclus par écrit, le comportement effectif des parties et la capacité des parties à gérer et assumer les risques de paiement correspondants. En particulier, le montant des paiements prévus devrait tenir compte de la valeur temps de l'argent et des éléments de risque propres aux conditions de paiement choisies. Par exemple, si une technique d'évaluation est appliquée pour calculer la valeur forfaitaire actualisée d'un actif incorporel transféré et qu'un contribuable choisit un paiement conditionné aux ventes à venir, le taux d'actualisation employé pour convertir 
l'évaluation forfaitaire en un flux de paiements conditionnés par les ventes pendant la durée d'utilité de l'actif transféré devrait tenir compte, d'une part, du risque accru pour le cédant si les ventes ne se réalisent pas et donc si les paiements n'interviennent pas et, d'autre part, des conséquences, en termes de valeur temps de l'argent, du report des paiements sur les années suivantes.

\section{[D.3. Détermination du prix de pleine concurrence lorsque l'évaluation est très incertaine au moment de la transaction}

Selon le Plan d'action concernant le BEPS, un volet des travaux à venir portera sur la fixation des prix de transfert pour les actifs incorporels difficiles à valoriser. Ces travaux s'appuieront notamment sur un examen approfondi du texte existant et de l'approche proposée à ce sujet dans les Principes applicables en matière de prix de transfert. En conséquence, aux fins de cette version de travail, le texte figurant dans les Principes de 2010 à propos des actifs incorporels difficiles à valoriser a été conservé, y compris celui des exemples 31 à 33. Des travaux importants devraient cependant être consacrés à ce sujet au cours des prochains mois.

6.178 Comme indiqué au début de cette section, les biens incorporels sont parfois très spécifiques, de sorte qu'il est difficile de trouver des éléments de comparaison et, dans certains cas, d'évaluer le bien incorporel au moment où il fait l'objet d'une transaction entre entreprises associées. Lorsque l'évaluation du bien incorporel pouvant être effectuée au moment de la transaction est très incertaine, le problème qui se pose est de savoir comment déterminer le prix de pleine concurrence. La solution consiste, tant pour le contribuable que pour l'administration fiscale, à se référer à la manière dont des entreprises indépendantes auraient procédé dans des circonstances comparables pour tenir compte, dans la fixation du prix de la transaction, de l'incertitude entourant l'évaluation.

6.179 En fonction des faits et des circonstances, des entreprises indépendantes peuvent procéder de différentes manières pour faire face à ce problème de l'incertitude de l'évaluation lorsqu'elles fixent le prix d'une transaction. L'une des possibilités consiste à fixer le prix dès le départ sur la base des avantages escomptés (compte tenu des divers facteurs économiques). Pour déterminer les avantages escomptés, des entreprises indépendantes rechercheraient dans quelle mesure l'évolution future est envisageable et prévisible. Dans certains cas, des entreprises indépendantes jugeront que des prévisions d'avantages sont suffisamment fiables pour que 
le prix de la transaction soit fixé dès le départ en fonction de ces prévisions, sans se réserver le droit de procéder à des ajustements à l'avenir.

6.180 Dans d'autres cas, des entreprises indépendantes jugeront que la fixation du prix uniquement sur la base des avantages escomptés ne permet pas de se prémunir suffisamment contre les risques liés à la forte incertitude qui entoure l'évaluation du bien incorporel. Des entreprises indépendantes pourront alors opter pour des contrats de plus faible durée ou faire figurer dans leurs contrats des clauses de révision des prix, de façon à se prémunir contre des événements imprévisibles. Par exemple, le taux de la redevance pourra être relevé à mesure qu'augmentent les ventes du licencié.

6.181 On peut également imaginer que des entreprises indépendantes décident d'assumer jusqu'à un certain point le risque d'événements imprévisibles, en convenant toutefois de renégocier et de réviser le prix d'un commun accord dans l'éventualité d'un événement majeur et imprévu modifiant les hypothèses fondamentales retenues pour la fixation du prix. Par exemple, une telle renégociation pourrait être conforme à des conditions de pleine concurrence si un taux de redevance calculé en fonction des ventes d'un médicament breveté se révélait tout à fait excessif en raison de la mise au point inattendue d'un nouveau traitement très bon marché. Du fait du niveau excessif de la redevance, le licencié pourra n'avoir plus aucun intérêt à fabriquer le médicament, ce qui pourra justifier une renégociation du contrat (compte tenu de l'ensemble des faits et circonstances).

6.182 Lorsqu'une administration fiscale évalue le prix d'une transaction entre entreprises associées portant sur un actif incorporel dont l'évaluation était au départ très incertaine, elle doit se fonder sur les modalités qui auraient été adoptées dans des circonstances comparables par des entreprises indépendantes. Dès lors, dans le cas où des entreprises indépendantes auraient fixé le prix sur la base de prévisions données, l'administration fiscale devra évaluer le prix sur la même base. Dans ce cas, l'administration fiscale pourrait, par exemple, vérifier que les entreprises associées ont établi correctement les prévisions, compte tenu de l'ensemble des événements raisonnablement prévisibles, sans procéder de façon rétroactive.

6.183 L'administration fiscale éprouvera certes des difficultés, notamment si le contribuable ne se montre pas coopératif, pour déterminer quels sont les bénéfices qui étaient raisonnablement prévisibles au moment où a été conclue la transaction. On peut imaginer, par exemple, qu'un contribuable transfère un bien incorporel à une entreprise associée, fixe une redevance qui ne reflète pas la valeur ultérieure avérée du bien corporel et fasse valoir plus tard qu'il n'était pas possible, au moment du transfert, de 
prévoir le succès qu'a eu le produit. En pareil cas, l'administration fiscale, au vu des événements ultérieurs, pourra être conduite à s'interroger sur la façon dont auraient procédé des entreprises indépendantes en fonction des informations raisonnablement disponibles au moment de la transaction. Il faudra en particulier se demander si les entreprises associées avaient l'intention d'établir et ont effectivement établi des prévisions que des entreprises indépendantes auraient jugées adéquates, compte tenu des événements raisonnablement prévisibles et des risques d'événements imprévisibles, et si des entreprises indépendantes auraient veillé à se prémunir par des moyens supplémentaires contre le risque lié à l'incertitude entourant l'évaluation.

6.184 Dans le cas où des entreprises indépendantes auraient opté pour une clause de révision des prix, dans des circonstances comparables l'administration fiscale doit avoir la possibilité de déterminer le prix sur la base d'une telle clause. De même, dans le cas où des entreprises indépendantes auraient jugé imprévisibles des événements ultérieurs si fondamentaux que leur survenance aurait abouti à une renégociation pour l'avenir du prix fixé pour une transaction, ces événements devraient également conduire à modifier le prix d'une transaction comparable entre entreprises associées.

6.185 Bien entendu, il pourra s'écouler plusieurs années avant qu'une administration fiscale procède à la vérification d'une déclaration d'impôt. L'administration fiscale aura alors le droit d'ajuster le prix pour tous les exercices précédant le moment où a lieu la vérification, sur la base des informations que des entreprises indépendantes auraient utilisées dans des circonstances comparables pour fixer le prix.]

\section{D.4. Instructions supplémentaires pour les transactions portant sur l'utilisation d'actifs incorporels en lien avec la vente de biens ou la fourniture de services}

6.186 Cette section fournit des instructions supplémentaires pour l'application des principes exposés dans les chapitres I à III lorsqu'une partie à la transaction contrôlée, ou les deux, utilisent des actifs incorporels en lien avec la vente de biens ou la fourniture de services sans procéder à un transfert d'actifs incorporels ou de droits sur des actifs incorporels. Lorsqu'elle porte sur des actifs incorporels, l'analyse de prix de transfert doit étudier avec soin les effets de l'existence de ces actifs sur les prix et les autres conditions des transactions contrôlées. 
(i) Les actifs incorporels comme facteur de comparabilité des transactions portant sur l'utilisation d'actifs incorporels

6.187 Les principes généraux énoncés aux paragraphes 1.33-1.63 et au chapitre III s'appliquent également aux analyses de comparabilité qui visent des transactions portant sur des actifs incorporels en lien avec une transaction contrôlée relative à la vente de biens ou à la fourniture de services. Cependant, le fait que les actifs concernés soient des actifs incorporels peut soulever des défis en matière de comparabilité.

6.188 Aux fins d'une analyse des prix de transfert, lorsque la méthode de fixation des prix de transfert la plus appropriée est celle du prix de revente, du coût majoré ou la méthode transactionnelle de la marge nette, la partie à la transaction qui présente le degré de complexité moindre est souvent retenue comme partie testée. Dans de nombreux cas, les prix de pleine concurrence ou la part des bénéfices attribuée à la partie testée peuvent être déterminés sans qu'il soit nécessaire d'évaluer les actifs incorporels utilisés en lien avec la transaction. Cette situation se présente généralement lorsque seule la partie non testée utilise des actifs incorporels. Dans certains cas toutefois, la partie testée, malgré le degré de complexité moindre de ses activités, peut utiliser de fait des actifs incorporels. De même, les parties à des transactions sur le marché libre potentiellement comparables peuvent utiliser des actifs incorporels. Dès lors qu'une telle situation se présente, il devient nécessaire d'examiner les actifs incorporels utilisés par la partie testée ou par les parties aux transactions sur le marché libre potentiellement comparables, les actifs incorporels constituant un facteur de comparabilité aux fins de l'analyse.

6.189 Par exemple, une partie testée qui assure la commercialisation et la distribution de biens achetés dans le cadre de transactions contrôlées peut avoir mis au point des actifs incorporels relevant du domaine de la commercialisation dans les zones géographiques où elle conduit ses activités, comme des listes de clients, des relations avec la clientèle ou des données relatives aux clients. Elle peut aussi avoir développé des savoir-faire en matière de logistique, développé des logiciels ou d'autres outils qui lui procurent des avantages dans l'exercice de ses activités de distribution. Les effets de ces actifs incorporels sur la rentabilité de la partie testée devraient être pris en compte lors de l'analyse de comparabilité.

6.190 Il convient cependant de souligner que bien souvent, lorsque la partie testée utilise de tels actifs incorporels, les parties aux transactions comparables sur le marché libre disposent elles aussi d'actifs de même type. Ainsi, dans le cas d'une entreprise de distribution, il est probable qu'une 
entité indépendante exerçant des activités de distribution dans le même secteur et sur le même marché que la partie testée connaisse des clients potentiels et soit entrée en relation avec eux, qu'elle ait recueilli des données concernant ces clients, qu'elle dispose de ses propres systèmes logistiques efficaces ou détienne dans d'autres domaines encore des actifs incorporels similaires à ceux de la partie testée. Dans ce cas, le degré de comparabilité peut être suffisamment élevé pour que les prix acquittés ou les marges engrangées par les entreprises potentiellement comparables constituent un indicateur approprié pour déterminer une rémunération de pleine concurrence des fonctions que la partie testée exerce et des actifs incorporels qu'elle possède.

6.191 Lorsque la partie testée et l'entreprise potentiellement comparable détiennent des actifs incorporels comparables, ceux-ci ne sont pas des actifs incorporels uniques et de valeur au sens du paragraphe 6.17 et aucun ajustement de comparabilité n'est par conséquent nécessaire au titre des actifs incorporels. Dans ce cas, le comparable potentiel représente le mieux la contribution des actifs incorporels de la partie testée aux bénéfices de celle-ci. Toutefois, si des actifs incorporels uniques et de valeur sont détenus et utilisés soit par la partie testée, soit par l'entreprise potentiellement comparable, il peut être nécessaire de réaliser des ajustements de comparabilité, voire de sélectionner une autre méthode de prix de transfert. Dans ce cas de figure, les principes exposés aux sections D.2.(i) à D.2.(iv) s'appliquent à l'évaluation de la comparabilité des actifs incorporels.

6.192 Il convient que les contribuables et les administrations fiscales fassent preuve de retenue lorsqu'ils rejettent des comparables potentiels du fait de l'utilisation d'actifs incorporels par les parties à des transactions potentiellement comparables ou par la partie testée. En règle générale, des comparables potentiels ne devraient pas être rejetés parce que l'existence d'actifs incorporels non spécifiés ou d'une survaleur importante a été avancée. Si des transactions ou des entreprises identifiées sont par ailleurs comparables, elles peuvent fournir le meilleur indicateur disponible pour fixer le prix de pleine concurrence, indépendamment de l'existence d'actifs incorporels relativement peu importants utilisés soit par la partie testée, soit par les parties aux transactions potentiellement comparables. Le rejet de transactions potentiellement comparables devrait être fondé sur l'existence et l'utilisation d'actifs incorporels non comparables seulement lorsque ces derniers peuvent être identifiés de façon claire et distincte et qu'il s'agit manifestement d'actifs uniques et de valeur. 
(ii) Détermination de prix de pleine concurrence pour des transactions portant sur l'utilisation d'actifs incorporels en lien avec la vente de biens ou la fourniture de services

6.193 Les principes énoncés dans les chapitres I à III s'appliquent à la fixation des prix de pleine concurrence pour les transactions portant sur l'utilisation d'actifs incorporels en lien avec la vente de biens ou la fourniture de services. On distingue généralement deux types de situations. Dans le premier cas, l'analyse de comparabilité, notamment l'analyse fonctionnelle, conclut à l'existence de comparables suffisamment fiables pour déterminer les conditions de pleine concurrence pour la transaction en utilisant une méthode de prix de transfert fondée sur des comparables. Dans le second cas, l'analyse de comparabilité, notamment l'analyse fonctionnelle, ne permet pas d'identifier de manière fiable des transactions comparables sur le marché libre, ce qui découle souvent directement de l'utilisation par une partie à la transaction, ou par les deux parties, d'actifs incorporels uniques et de valeur. Les approches applicables dans les deux cas afin de déterminer les prix de transfert sont décrites dans les paragraphes suivants.

\section{Situations où des comparables fiables existent}

$6.194 \quad$ Il est fréquent que des comparables fiables puissent être identifiés malgré l'utilisation d'actifs incorporels par l'une au moins des parties à une transaction contrôlée de vente de biens ou de fourniture de services. Selon les circonstances du cas d'espèce, l'une ou l'autre des cinq méthodes de prix de transfert de l'OCDE décrites au chapitre II pourra être la plus appropriée si la transaction s'accompagne de l'utilisation d'actifs incorporels en lien avec une transaction contrôlée de vente de biens ou de fourniture de services et si des comparables fiables existent.

6.195 Lorsque la partie testée n'utilise pas d'actifs incorporels uniques et de valeur, et lorsque des comparables fiables peuvent être identifiés, les prix de pleine concurrence peuvent souvent être établis en employant une méthode unilatérale, notamment les méthodes du prix comparable sur le marché libre, du prix de revente, du coût majoré ou la méthode transactionnelle de la marge nette. Dans ce cas, les indications présentées dans les chapitres I à III sont généralement suffisantes aux fins de la détermination des prix de pleine concurrence, sans qu'il y ait lieu d'examiner en détail la nature des actifs incorporels utilisés par l'autre partie à la transaction.

6.196 Il convient de se référer aux principes décrits aux sections D.2.(i) à D.2.(iv) du présent chapitre pour déterminer si l'utilisation des actifs 
incorporels par la partie testée retire toute leur fiabilité aux transactions comparables sur le marché libre qui ont été identifiées ou rend nécessaire la réalisation d'ajustements de comparabilité. Le recours à des ajustements de comparabilité ou le choix d'une méthode de prix de transfert moins dépendante des transactions comparables sur le marché libre ne s'imposent que si les actifs incorporels utilisés par la partie testée sont des actifs uniques et de valeur. Lorsque les actifs incorporels utilisés par la partie testée sont d'une autre nature, les prix versés ou perçus, les marges et revenus obtenus par les parties lors de transactions comparables sur le marché libre peuvent fournir un indicateur fiable pour déterminer les conditions de pleine concurrence.

6.197 Lorsque des ajustements de comparabilité s'imposent du fait des différences entre les actifs incorporels utilisés par la partie testée dans le cadre d'une transaction contrôlée et les actifs incorporels utilisés par une partie dans le cadre d'une transaction sur le marché libre potentiellement comparable, le calcul d'ajustements de comparabilité fiables peut soulever des questions factuelles auxquelles il est difficile de répondre. Il convient alors de procéder à un examen approfondi des faits et circonstances du cas d'espèce et des données disponibles concernant les effets des actifs incorporels sur les prix et les bénéfices. Lorsqu'une différence de nature entre différents actifs incorporels a manifestement un effet sur les prix mais ne peut faire l'objet d'une estimation fiable, il peut s'avérer nécessaire de choisir une méthode de prix de transfert moins dépendante de l'identification de comparables fiables.

6.198 Il convient également de souligner que, dans le cas d'une utilisation d'actifs incorporels en lien avec une transaction contrôlée de vente de biens ou de services, les différences de nature entre les actifs incorporels utilisés ne sont pas les seules raisons justifiant des ajustements de comparabilité. En particulier, des ajustements de comparabilité peuvent s'imposer du fait de différences existant entre les marchés, les avantages de localisation, les stratégies opérationnelles, le collectif de travail, les synergies d'entreprise et d'autres facteurs similaires. Si ces facteurs ne peuvent être qualifiés d'actifs incorporels au sens donné à ce terme dans la section A.1 du présent chapitre, ils peuvent cependant avoir des effets importants sur les prix de pleine concurrence dans des situations caractérisées par l'utilisation d'actifs incorporels.

$$
\text { Situations où il n'existe pas de comparables fiables }
$$

6.199 Lorsqu'aucune transaction sur le marché libre comparable de manière fiable n'a pu être identifiée, la méthode transactionnelle du partage des bénéfices peut être utilisée pour définir une répartition conforme au 
principe de pleine concurrence des bénéfices générés par les ventes de produits ou les fournitures de services assorties de l'utilisation d'actifs incorporels. La méthode transactionnelle du partage des bénéfices peut notamment être utilisée lorsque les deux parties à la transaction apportent des contributions uniques et de grande valeur à la transaction. Voir à ce propos le paragraphe 2.110 .

6.200 Les paragraphes 2.109-2.146 contiennent des indications à prendre en compte pour mettre en œuvre la méthode transactionnelle du partage des bénéfices. Ces indications s'appliquent pleinement aux situations caractérisées par l'utilisation d'actifs incorporels en lien avec la vente de produits ou la fourniture de services dans le cadre de transactions contrôlées.

6.201 Lors de l'application d'une méthode de partage des bénéfices à un cas d'espèce caractérisé par l'utilisation d'actifs incorporels, il faut veiller à identifier les actifs incorporels concernés et à étudier de quelle manière ils contribuent à la création de valeur et évaluer avec soin les autres fonctions exercées qui génèrent des revenus, les actifs utilisés et les risques assumés. Pour pouvoir être mise en œuvre de manière fiable, une méthode de partage des bénéfices ne pourra reposer uniquement sur des affirmations imprécises concernant l'existence et l'utilisation d'actifs incorporels.

6.202 Lorsque les circonstances le justifient, des méthodes de fixation des prix de transfert ou des méthodes d'évaluation qui ne dépendent pas de l'identification de transactions sur le marché libre qui soient comparables et fiables peuvent également être utilisées pour définir les conditions de pleine concurrence pour une vente de produits ou fourniture de services lors de laquelle des actifs incorporels sont utilisés en lien avec la transaction. La solution alternative retenue devrait tenir compte de la nature des biens ou services fournis et de la contribution à la création de valeur apportée par les actifs incorporels et par les autres facteurs pertinents. 


\section{Notes}

1. Tel qu'il est utilisé dans ce paragraphe, le terme «actif financier » désigne tout actif qui est de la trésorerie, un instrument de fonds propres, un droit contractuel ou une obligation contractuelle de recevoir de la trésorerie ou un autre actif financier, ou d'échanger des actifs ou passifs financiers, ou un instrument dérivé. On peut citer à titre d'exemples les obligations, les dépôts bancaires, les actions et autres titres de participation, les contrats à terme standardisés ou de gré à gré, ainsi que les contrats d'échange.

2. Tel qu'elle est utilisée ici, la notion d'« exploitation d'un actif incorporel» recouvre à la fois le transfert de l'actif incorporel considéré ou des droits sur cet actif incorporel et son utilisation dans le cadre d'opérations commerciales.

3. Tel qu'elle est utilisée dans la présente section B, la notion d'« utilisation d'actifs » recouvre la contribution apportée sous forme de financement et/ou d'autres formes de capital à la mise au point, à l'amélioration, à l'entretien, à la protection ou à l'exploitation d'actifs incorporels. Voir le paragraphe 6.59 .

4. Le Plan d'action concernant le BEPS invite explicitement à conduire des travaux concernant les actifs incorporels difficiles à valoriser et l'application des méthodes de partage des bénéfices. Les dispositions des paragraphes 6.145-6.149 seront réexaminées dans le cadre de ces travaux.

5. Les évaluations financières fondées sur des prévisions s'appuient souvent sur des flux de trésorerie prévisionnels. Les estimations fondées sur les droits constatés, comme celles réalisées à des fins comptables ou fiscales, reflètent parfois mal l'échéancier des flux de trésorerie, ce qui peut conduire à des résultats différents selon que l'approche est fondée sur les revenus ou sur les flux de trésorerie. Toutefois, plusieurs arguments conduisent à considérer que, dans certains cas, les prévisions fondées sur les revenus donnent en pratique des résultats plus fiables que celles fondées sur les flux de trésorerie aux fins de la fixation des prix de transfert. Dans les tous cas, cependant, on veillera à ce que les estimations fondées sur les revenus ou sur les flux de trésorerie soient utilisées de manière cohérente et lorsque les circonstances le justifient. Par conséquent, les références à une approche fondée sur les flux de trésorerie 
renvoient plus largement aux estimations fondées sur les revenus et sur les flux de trésorerie, appliquées à bon escient. 

Les dispositions de l'annexe au chapitre VI des Principes applicables en matière de prix de transfert sont supprimées dans leur intégralité et remplacées par celles qui suivent.

\author{
Annexe au chapitre VI
}

\title{
Exemples d'application des instructions concernant les considérations particulières applicables aux actifs incorporels
}

\section{[Exemple 1}

1. Première est la société mère d'un groupe multinational. La société $\mathrm{S}$ est une filiale détenue intégralement par Première et membre du groupe Première. Première finance et exerce des fonctions courantes de recherche-développement qui étayent ses activités industrielles et commerciales. Lorsque ses fonctions de recherche-développement débouchent sur des inventions brevetables, il est d'usage que le groupe Première attribue tous les droits sur ces inventions à la société $\mathrm{S}$, afin de centraliser et de simplifier l'administration des brevets à l'échelle mondiale. Tous les brevets déposés sont détenus et maintenus en vigueur sont au nom de la société $\mathrm{S}$.

2. La société $\mathrm{S}$ emploie trois juristes chargés de l'administration de ses brevets et n'a pas d'autre employé. La société $\mathrm{S}$ ne réalise ni ne contrôle aucune des activités de recherche-développement du groupe Première. La société S n'a aucun personnel technique de recherche-développement, et ne prend aucunement en charge les dépenses de recherche-développement du groupe Première. La direction de Première prend les décisions clés relatives à la défense des brevets, après avoir consulté les employés de la société $\mathrm{S}$. C'est la direction de Première, et non les employés de la société $\mathrm{S}$, qui contrôle toutes les décisions concernant la concession sous licence des brevets du groupe, que ce soit à des entreprises indépendantes ou associées.

3. Lors de chaque attribution de droits par Première à la société $\mathrm{S}$, celle-ci effectue un paiement symbolique de 100 EUR à Première correspondant à l'attribution des droits sur une invention brevetable et, à titre 
de condition spécifique de cette attribution, accorde simultanément à Première une licence exclusive, sans redevance, d'exploitation du brevet considéré, assorti de droits illimités de concession de sous-licence, pour toute la durée de vie du brevet devant être enregistré. Les paiements symboliques effectués par la société $\mathrm{S}$ au bénéfice de Première sont uniquement destinés à satisfaire des prescriptions techniques du droit des contrats liées aux attributions et, aux fins de cet exemple, il est supposé qu'ils ne correspondent pas à une rémunération de pleine concurrence des droits attribués sur des inventions brevetables. Première utilise ces inventions brevetées pour la fabrication et la vente de ses produits dans le monde entier et, de temps en temps, cède des droits sur ces brevets par le biais de sous-licences accordées à des tiers. La société $\mathrm{S}$ ne fait aucun usage commercial de ces brevets, et n'est pas en droit de le faire en vertu de l'accord de licence conclu avec Première.

4. Aux termes de cet accord, Première exerce toutes les fonctions liées à la mise au point, à l'amélioration, à l'entretien, à la protection et à l'exploitation des actifs incorporels considérés, exception faite des services d'administration des brevets. Première fournit et utilise tous les actifs associés à la mise au point et à l'exploitation de ces actifs incorporels, et assume la totalité ou la quasi-totalité des risques associés à ces actifs incorporels. Première devrait donc avoir droit à l'essentiel des revenus tirés de l'exploitation des actifs incorporels considérés. Les administrations fiscales pourraient parvenir à une solution appropriée en termes de prix de transfert en déterminant la véritable nature des dispositions conclues entre Première et la société $\mathrm{S}$. Suivant les faits du cas d'espèce, il pourrait être établi que, considérés dans leur ensemble, l'attribution purement formelle des droits à la société $\mathrm{S}$ et la rétrocession simultanée de l'intégralité des droits exploitation à Première correspond en substance à un accord de services d'administration des brevets entre Première et la société $\mathrm{S}$. Un prix de pleine concurrence serait établi pour ces services d'administration des brevets, et Première conserverait ou se verrait attribuer le solde des revenus tirés par le groupe multinational de l'exploitation de ces brevets.

\section{Exemple 2}

5 .

Les faits relatifs à la mise au point et au contrôle des inventions brevetables considérées sont les mêmes que dans l'exemple 1. Néanmoins, au lieu de rétrocéder à une licence exclusive d'utilisation perpétuelle de ses brevets à Première, la société $\mathrm{S}$, agissant sous la direction et le contrôle de Première, accorde des licences d'exploitation de ses brevets à des entreprises associées et indépendantes dans le monde entier en contrepartie de redevances périodiques. Aux fins du présent exemple, il est supposé que les 
redevances payées à la société $\mathrm{S}$ par les entreprises associées sont toutes conformes au principe de pleine concurrence.

6. La société $\mathrm{S}$ est le propriétaire légal des brevets. Néanmoins, ses contributions à la mise au point, à l'amélioration, à l'entretien, à la protection et à l'exploitation de ces brevets sont limitées aux activités de ses trois employés consistant à faire enregistrer et maintenir en vigueur les brevets. Les employés de la société $\mathrm{S}$ ne contrôlent pas les opérations de concession de licence relatives aux brevets et ne participe pas non plus à ces transactions. Dans ces circonstances, la société $\mathrm{S}$ a uniquement droit à une rémunération des fonctions qu'elle exerce. Sur la base d'une analyse des fonctions exercées, des actifs utilisés et des risques assumés respectivement par Première et la société $S$ dans le cadre de la mise au point, de l'amélioration, de l'entretien, de la protection et de l'exploitation des actifs incorporels considérés, la part des revenus tirés par la société $\mathrm{S}$ de ses accords de licence qu'elle devrait être en droit de conserver ou de se voir attribuer in fine devrait se limiter à la rémunération de pleine concurrence de ses fonctions d'enregistrement des brevets.

7. Comme dans l'exemple 1, la véritable nature de l'accord en place est un contrat de services d'administration de brevets. Il est possible de garantir qu'un prix de transfert approprié soit appliqué en veillant à ce que le montant versé par la société $\mathrm{S}$ en contrepartie de l'attribution des droits sur les brevets corresponde effectivement aux fonctions exercées, aux actifs utilisés et aux risques assumés respectivement par Première et la société $\mathrm{S}$. Suivant cette approche, la rémunération ex ante due à Première au titre des inventions brevetables est égale aux recettes anticipées de la cession des droits de licence de la société $\mathrm{S}$, diminuées d'une rémunération appropriée des fonctions exercées par la société $\mathrm{S}$. La question de la répartition des éventuels revenus ex post n'est pas abordée dans cet exemple.

\section{Exemple 3}

8. Les faits sont les mêmes que dans l'exemple 2. Néanmoins, après avoir concédé sous licence les brevets à des entreprises associées et indépendantes pendant quelques années, la société $\mathrm{S}$, agissant là encore sous la direction et le contrôle de Première, vend ces brevets à une entreprise indépendante à un prix reflétant l'augmentation de la valeur des brevets au cours de la période durant laquelle la société $\mathrm{S}$ en était le propriétaire légal. Les fonctions exercées par la société $S$ tout au long de la période pendant laquelle elle était le propriétaire légal des brevets se limitaient aux activités d'enregistrement de ces brevets décrites dans les exemples 1 et 2 .

9. Dans ces circonstances, les revenus de la société $\mathrm{S}$ devraient être les mêmes que dans l'exemple 2. Elle doit être rémunérée pour les fonctions 
d'enregistrement qu'elle exerce, mais n'a droit par ailleurs à aucune part des revenus tirés de l'exploitation des actifs incorporels considérés, notamment des revenus provenant de la cession de ces actifs.

\section{Exemple 4}

10. Les faits relatifs à la mise au point des brevets et à l'attribution initiale de ces brevets à la société $\mathrm{S}$ sont les mêmes que ceux décrits dans l'exemple 3. Néanmoins, nous supposerons aux fins de cet exemple que le prix payé par la société $S$ en contrepartie des brevets était un prix de pleine concurrence correspondant avec précision aux revenus escomptés de l'exploitation des brevets au moment de leur attribution à la société $\mathrm{S}$.

11. À la suite des attributions, la société $\mathrm{S}$ a concédé sous licence les brevets à des entreprises indépendantes pour quelques années. Contrairement à l'exemple 1 , la société $\mathrm{S}$ a en l'occurrence des employés qui peuvent prendre toutes les décisions de gestion relatives à son portefeuille de brevets. Toutes les décisions concernant le programme de concession de licences ont été prises par les employés de la société $\mathrm{S}$, toutes les négociations avec les preneurs de licences ont été menées par les employées de la société $\mathrm{S}$, et ceux-ci se sont assurés que les titulaires de licences indépendants respectaient les conditions desdites licences. Par la suite, la valeur des brevets augmente sensiblement en raison de circonstances externes imprévues au moment de l'attribution des brevets à la société $\mathrm{S}$. Celle-ci vend alors les brevets à un acquéreur avec lequel elle n'a aucun lien, à un prix supérieur à celui initialement payé par la société $\mathrm{S}$ à Première pour ces brevets. Les employés de la société $\mathrm{S}$ prennent toutes les décisions concernant la vente de ces brevets, négocient les conditions de la vente, et gèrent et contrôlent à tous égards la cession des brevets.

12. Dans ces circonstances, la société $\mathrm{S}$ est en droit de conserver les produits de la vente, y compris les montants attribuables à l'augmentation de la valeur des brevets résultant des circonstances externes imprévues.

\section{Exemple 5}

13. Les faits sont les mêmes que dans l'exemple 4, si ce n'est qu'au lieu d'augmenter, la valeur des brevets diminue pendant leur période de détention par la société $\mathrm{S}$ en raison de circonstances externes imprévues. Dans ces circonstances, la société $\mathrm{S}$ est en droit de conserver les produits de la vente, ce qui implique qu'elle subira la perte connexe. 


\section{Exemple 6}

14. Les faits sont les mêmes que dans l'exemple 4, si ce n'est que l'augmentation de la valeur des brevets résulte des efforts déployés par Première, qui a lancé, financé et géré une campagne de commercialisation intensive et fructueuse sur de nouveaux marchés pour les produits couverts par les brevets considérés. Cette campagne fructueuse de commercialisation se traduit par une hausse sensible des redevances reçues par la société $\mathrm{S}$. Dans ces circonstances, la société $S$ doit rémunérer Première pour sa contribution à l'accroissement de la valeur des brevets.

\section{Exemple 7}

15. Au cours de l'année 1, un groupe multinational composé de la société A (domiciliée dans le pays A) et d'une société B (domiciliée dans le pays B) décide de mettre au point un actif incorporel, dont on escompte une rentabilité élevée sur la base des actifs incorporels dont dispose déjà la société $\mathrm{B}$, de ses résultats et de l'expérience de son personnel de recherchedéveloppement. La mise au point de cet actif incorporel devrait s'étaler sur une période de 5 ans, après laquelle son exploitation commerciale deviendra possible. Si la phase de mise au point est couronnée de succès, cet actif incorporel devrait avoir de la valeur pendant une période totale d'exploitation de 10 ans. En vertu de l'accord de mise au point conclu entre la société $\mathrm{A}$ et la société $\mathrm{B}$, cette dernière accomplira et contrôlera toutes les activités liées à la mise au point, à l'entretien et à l'exploitation de l'actif incorporel considéré. La société $\mathrm{A}$, quant à elle, fournira la totalité des financements associés à la mise au point de cet actif incorporel (sachant que l'on table sur des coûts de mise au point de 100 millions USD par an pendant 5 ans), et deviendra le propriétaire légal de cet actif incorporel. Une fois mis au point, cet actif incorporel devrait déboucher sur des bénéfices de 550 millions USD par an (au cours des années 6 à 15). La société B concèdera sous licence l'actif incorporel de la société $\mathrm{A}$ et versera des paiements conditionnels à la société $\mathrm{A}$ pour le droit d'utiliser cet actif incorporel, fondés sur les revenus de preneurs de licences présentés comme comparables. Une fois effectués les paiements conditionnels prévus, il restera à la société $\mathrm{B}$ un revenu anticipé de 200 millions USD par an tiré de la vente des produits fondés sur cet actif incorporel.

16. Dans le cadre d'une analyse fonctionnelle de ces dispositions, l'administration fiscale du pays B évalue les fonctions exercées, les actifs utilisés et fournis ainsi que les risques assumés par la société A et la société B. Elle conclut au terme de cette analyse que la contribution de la société A dans le cadre de ces dispositions se limite au financement de la 
recherche-développement, y compris la prise en charge du risque inhérent à ce financement. La société A exerce, par le biais de son propre personnel, toutes les fonctions escomptées d'une entité indépendante finançant un projet de recherche-développement; elle analyse notamment l'actif incorporel en question et les bénéfices escomptés de cet investissement, évalue le risque de financement $-\mathrm{y}$ compris le risque qu'un investissement supplémentaire soit nécessaire pour mener à bien le projet - et la capacité de la société $\mathrm{A}$ de prendre ce risque, et prend les décisions relatives à la prise en charge, à la couverture ou à l'atténuation de ce risque. Compte tenu des contributions de la société $\mathrm{A}$, ainsi que des solutions de remplacement réalistes dont disposent les sociétés $\mathrm{A}$ et $\mathrm{B}$, il est établi que la rémunération escomptée de la société A devrait être un taux de rendement anticipé corrigé des risques appliqué à son engagement de financement. Supposons que celui-ci soit évalué à 110 millions USD par an (pour les années 6 à 15) ${ }^{1}$. En conséquence, la société $\mathrm{B}$ aurait droit à la totalité des revenus anticipés restants une fois déduit le rendement anticipé pour la société $A$, soit 440 millions USD par an (550 millions USD moins 110 millions USD), et non 200 millions USD par an comme indiqué par le contribuable. (Sur la base de l'analyse fonctionnelle approfondie réalisée et de l'application de la méthode la plus appropriée, le contribuable a choisi de manière incorrecte la société B comme partie testée, au lieu de la société A.) La société A aurait donc droit à $20 \%$ des bénéfices anticipés, et la société $\mathrm{B}$ à $80 \%$.

17. Il faudrait ensuite faire correspondre les revenus anticipés attribués aux sociétés $\mathrm{A}$ et $\mathrm{B}$ et les dispositions contractuelles conclues. Autrement dit, les dispositions contractuelles conditionnelles (telles que les paiements au titre des licences) devraient être déterminées de manière que les bénéfices anticipés attribués aux deux sociétés s'établissent à 440 millions USD par an pour la société $\mathrm{B}$ et à 110 millions USD par an pour la société A. Bien que la société A soit le propriétaire légal de l'actif incorporel considéré, sa contribution au financement de la recherchedéveloppement et les risques associés à ce financement lui donnent droit à $20 \%$ seulement des bénéfices anticipés. Les bénéfices effectifs des sociétés $\mathrm{A}$ et $\mathrm{B}$ seront probablement supérieurs ou inférieurs à leurs bénéfices anticipés. Le cas échéant, les différences entre bénéfices effectifs et anticipés seront attribuées à la société $\mathrm{A}$ et/ou à la société $\mathrm{B}$ suivant les principes énoncés aux paragraphes 6.66 et 6.67.$]$

\section{Exemple 8}

18. Primero est la société mère d'un groupe multinational présent dans le secteur pharmaceutique et exerce des activités dans le pays M. Primero met au point des brevets et d'autres actifs incorporels liés au produit $\mathrm{X}$ et enregistre ces brevets dans des pays du monde entier. 
19. Primero conserve la filiale qu'elle détient intégralement dans le pays $\mathrm{N}$, la société $\mathrm{S}$, pour distribuer le produit $\mathrm{X}$ dans toute l'Europe et au Moyen-Orient sur la base d'une prise en charge limitée des risques. L'accord de distribution prévoit que Primero, et non la société S, doit assumer le risque de rappel du produit et le risque découlant de la responsabilité du fait du produit, et que Primero aura droit à la totalité des bénéfices (ou pertes) résultant de la vente du produit $\mathrm{X}$ sur le territoire considéré après avoir versé à la société $\mathrm{S}$ la rémunération convenue pour ses fonctions de distribution. En vertu du contrat, la société $S$ achète le produit $X$ à Primero et le revend à des clients indépendants se trouvant dans divers pays situés dans la zone géographique où elle exerce ses activités. Dans l'exercice de ses fonctions de distribution, la société $\mathrm{S}$ se conforme à toutes les prescriptions réglementaires en vigueur.

20. Au cours des trois premières années d'activité, la société $\mathrm{S}$ tire de ses fonctions de distribution des revenus qui correspondent à la prise en charge limitée des risques qui les caractérisent et aux conditions du contrat de distribution. Ses revenus reflètent le fait que c'est Primero, et non la société $\mathrm{S}$, qui est en droit de conserver les revenus tirés de l'exploitation des actifs incorporels liés au produit $\mathrm{X}$. Après trois années d'activité, il devient manifeste que le produit $X$ a de graves effets secondaires chez un pourcentage important des patients utilisant ce produit, et il devient nécessaire de rappeler ce produit et de le retirer du marché. Ce rappel représente un coût considérable pour la société S. Primero ne rembourse pas à la société $\mathrm{S}$ ces frais liés au rappel du produit, ni les coûts liés aux demandes d'indemnisation au titre de la responsabilité du fait du produit.

21. Dans ces circonstances, on observe une incohérence entre le droit prétendu de Primero aux revenus tirés de l'exploitation des actifs incorporels liés au produit $\mathrm{X}$ et le fait qu'elle n'assume pas les coûts associés aux risques étayant cette prétention. Un ajustement de prix de transfert serait justifié pour remédier à cette incohérence. Pour déterminer l'ajustement approprié à effectuer, il faudrait cerner la véritable transaction ayant eu lieu entre les parties considérées, en appliquant les dispositions du paragraphe 1.53. Ce faisant, il conviendrait de prendre en compte les risques assumés par chacune des parties sur la base de leur comportement au cours de la période couverte par l'accord, du contrôle exercé sur les risques par Primero et la société $\mathrm{S}$, de leurs capacités respectives d'assumer ces risques, ainsi que des autres faits pertinents. S'il est établi que la véritable nature de la relation entre les parties correspond à un accord de distribution assorti d'une prise en charge limitée des risques, l'ajustement le plus approprié consisterait sans doute à réaffecter les frais liés au rappel du produit et à la responsabilité du fait du produit de la société $\mathrm{S}$ à Primero. Par contre, s'il est établi sur la base de tous les faits pertinents que la véritable nature de la relation entre les 
parties correspond à une prise en charge de la responsabilité du fait du produit et du risque de rappel par la société $\mathrm{S}$, et si un prix de pleine concurrence peut être identifié sur la base de l'analyse de comparabilité, on pourrait réviser à la hausse la marge de distribution de la société $\mathrm{S}$ pour toutes les années considérées de manière à prendre en compte la véritable répartition des risques entre les parties. Dans la mesure où les risques et les fonctions liées au contrôle des risques doivent généralement se correspondre, cette seconde éventualité est peu probable.

\section{Exemple 9}

22. Primair, entreprise résidente du pays $X$, fabrique des montres qui sont commercialisées dans de nombreux pays du monde sous le nom commercial et la marque de fabrique R. Primair est le titulaire du nom commercial et de la marque de fabrique $\mathrm{R}$. Le nom $\mathrm{R}$ est très connu dans les pays où ces montres sont vendues, et a acquis une valeur économique considérable sur ces marchés grâce aux efforts déployés par Primair. Les montres R n'ont jamais été commercialisées dans le pays $\mathrm{Y}$, cependant, et le nom $\mathrm{R}$ n'est pas connu sur le marché du pays $\mathrm{Y}$.

23. L'année 1, Primair décide d'entrer sur le marché du pays $Y$ et constitue en société une filiale qu'elle détient intégralement dans le pays $\mathrm{Y}$, la société $\mathrm{S}$, afin qu'elle y soit son distributeur. Parallèlement, Primair conclut avec la société $\mathrm{S}$ un accord à long terme de commercialisation et de distribution ne donnant pas lieu au paiement de redevances. En vertu de cet accord, la société $\mathrm{S}$ se voit accorder le droit exclusif de commercialiser et distribuer les montres portant la marque de fabrique $\mathrm{R}$ et utilisant le nom commercial $\mathrm{R}$ dans le pays $\mathrm{Y}$ pour une période de cinq ans, avec une option pour cinq années supplémentaires. La société $\mathrm{S}$ n'obtient de Primair aucun autre droit relatif au nom commercial et à la marque de fabrique $\mathrm{R}$, et il lui notamment interdit de réexporter les montres portant le nom commercial et la marque de fabrique $\mathrm{R}$. L'activité de la société $\mathrm{S}$ consiste exclusivement à commercialiser et distribuer des montres portant le nom commercial et la marque de fabrique R. Il est supposé que les montres $\mathrm{R}$ ne font pas partie d'un portefeuille de produits distribués par la société $\mathrm{S}$ dans le pays $\mathrm{Y}$. La société $\mathrm{S}$ ne procède à aucune opération de transformation, dans la mesure où elle importe dans le pays $\mathrm{Y}$ des montres sous emballage et prêtes à être vendues au consommateur final.

24. En vertu du contrat conclu entre Primair et la Société S, celle-ci achète les montres à Primair dans la monnaie du pays $\mathrm{Y}$, devient propriétaire des montres de marque et exerce les fonctions de distribution dans le pays $\mathrm{Y}$, prend en charge les coûts de possession (tels que le financement du stockage et des créances), et assume les risques correspondants (comme les 
risques de stock, de crédit et de financement). En vertu du contrat conclu entre Primair et la société S, celle-ci est tenue de jouer le rôle d'agent de commercialisation pour contribuer au développement du marché pour les montres R dans le pays $\mathrm{Y}$. La société $\mathrm{S}$ consulte Primair pour l'élaboration de la stratégie de commercialisation des montres R dans le pays Y. Primair définit le plan de commercialisation global en se fondant dans une large mesure sur l'expérience qu'elle a acquise dans d'autres pays, elle établit et approuve les budgets de commercialisation, et elle prend les décisions finales en matière de supports publicitaire, de positionnement de produit et de messages publicitaires clés. La société $\mathrm{S}$ est consultée sur les questions de publicité liées au marché local, contribue à l'exécution de la stratégie de commercialisation sous la direction de Primair, et fournit des évaluations de l'efficacité de divers éléments de cette stratégie de commercialisation. À titre de rémunération de ces activités d'aide à la commercialisation, la société $\mathrm{S}$ reçoit de Primair une commission fondée sur le niveau des dépenses de commercialisation qu'elle prend en charge et incluant un élément de bénéfice approprié.

25. Supposons aux fins de cet exemple que, sur la base d'une analyse de comparabilité approfondie, incluant une analyse fonctionnelle détaillée, il soit possible de conclure que le prix payé par la société $\mathrm{S}$ à Primair pour les montres $\mathrm{R}$ doit être analysé séparément de la rémunération reçue par la société $\mathrm{S}$ au titre des activités de commercialisation qu'elle réalise au nom de Primair. Supposons par ailleurs que sur la base des transactions comparables identifiées, il soit établi que le prix payé pour les montres est conforme au principe de pleine concurrence, et que ce prix permet à la société $\mathrm{S}$ d'obtenir grâce à la vente des montres une rémunération de pleine concurrence au titre des fonctions de distribution qu'elle exerce, des actifs qu'elle utilise et des risques qu'elle assume.

26. Au cours des années 1 à 3 , la société $\mathrm{S}$ lance une stratégie qui est conforme avec son accord avec Primaire selon lequel elle doit développer le marché pour les montres $\mathrm{R}$ dans le pays $\mathrm{Y}$. Ce faisant, la société $\mathrm{S}$ doit engager des dépenses de commercialisation. Conformément au contrat, Primair rembourse à la société $\mathrm{S}$ ces dépenses de commercialisation, augmentées d'une marge. À la fin de l'année 2, le nom commercial et la marque de fabrique $\mathrm{R}$ sont devenues bien établies dans le pays $\mathrm{Y}$. Il est établi que la rémunération obtenue par la société $\mathrm{S}$ au titre des activités de commercialisation réalisées au nom de Primair est conforme au principe de pleine concurrence, sur la base d'une comparaison avec celle versée à des agents publicitaires et de commercialisation identifiés et jugés comparables dans le cadre de l'analyse de comparabilité effectuée.

27. Dans ces circonstances, Primair est en droit de conserver, le cas échant, les revenus tirés de l'exploitation du nom commercial et de la 
marque de fabrique $\mathrm{R}$ sur le marché du pays $\mathrm{Y}$, diminués de la rémunération de pleine concurrence devant être versée à la société $\mathrm{S}$ pour ses fonctions, et aucun ajustement de prix de transfert ne s'impose compte tenu des circonstances.

\section{Exemple 10}

28. Aux fins de cet exemple, les faits sont identiques à ceux décrits dans l'exemple 9, hormis pour ce qui suit :

- En vertu du contrat conclu entre Primair et la société S, cette dernière est tenue d'élaborer et de mettre en œuvre un plan de commercialisation dans le pays $\mathrm{S}$ sans que Primair ne contrôle en détail les éléments particuliers de ce plan. La société S supporte les coûts des activités de commercialisation et assume certains des risques qui en découlent. Le contrat conclu entre Primair et la société $\mathrm{S}$ ne précise pas le montant des dépenses que la société $\mathrm{S}$ doit consacrer aux activités de commercialisation, mais prévoit seulement que la société $\mathrm{S}$ est liée par une obligation de moyens concernant la commercialisation des montres. Primair ne verse à la société $\mathrm{S}$ aucun remboursement au titre des dépenses engagées par cette dernière, ni de rémunération directe ou indirecte, et la rémunération de la société $\mathrm{S}$ est uniquement tirée des ventes de montres $\mathrm{R}$ auprès de clients tiers sur le marché du pays $\mathrm{Y}$. Une analyse fonctionnelle approfondie révèle que Primair exerce sur les activités de commercialisation de la société $\mathrm{S}$ un contrôle moins poussé que dans l'exemple 9 et que les éléments budgétaires et les actions détaillées du plan de commercialisation ne sont pas soumis à l'examen et à la validation de Primair. Par comparaison avec l'exemple 9, la société $\mathrm{S}$ assume cette fois des risques différents et ses modalités de rémunération sont différentes. Les dispositions contractuelles liant Primair et la société S diffèrent également et la société $\mathrm{S}$ assume dans l'exemple 10 des risques plus importants que dans l'exemple 9. La société $\mathrm{S}$ ne reçoit au titre de ses dépenses de commercialisation ni remboursement direct, ni commission versée séparément. L'unique transaction contrôlée entre Primair et la société S dans l'exemple 10 est le transfert des montres de marque. En conséquence, les activités de commercialisation de la société $\mathrm{S}$ sont uniquement rémunérées au travers de la vente de montres $\mathrm{R}$ auprès de clients tiers.

- Comme conséquence de ces différences, les montres sont vendues par Primair à la société $\mathrm{S}$ dans l'exemple 10 à un prix inférieur à 
celui pratiqué dans l'exemple 9. Du fait des différences observées lors de l'analyse fonctionnelle, par rapport à l'exemple 9, d'autres critères sont cette fois utilisés pour identifier des transactions comparables et pour réaliser des ajustements de comparabilité. Par comparaison avec l'exemple 9, la société $\mathrm{S}$ affiche un bénéfice total prévisionnel plus important dans l'exemple 10, où elle assume plus de risques et exerce des fonctions plus étendues.

29. Supposons qu'au cours des années 1 à 3 , la société $\mathrm{S}$ applique une stratégie conforme à son accord avec Primair et, à ce titre, mène à bien des fonctions de commercialisation et engage les dépenses correspondantes. $\mathrm{La}$ société $\mathrm{S}$ présente alors des dépenses opérationnelles élevées et des marges réduites durant les années 1 à 3 . À la fin de l'année 2, grâce aux efforts de la société $\mathrm{S}$, la marque de fabrique $\mathrm{R}$ et le nom commercial $\mathrm{R}$ sont bien établis dans le pays Y. Lorsque la société qui assure la commercialisation/distribution supporte effectivement les coûts et les risques associés à ses activités de commercialisation, la question de la répartition des bénéfices potentiels générés par ces activités est soulevée. Supposons que l'administration fiscale du pays $\mathrm{Y}$ ait conclus, sur le fondement de l'examen de distributeurs comparables, que, si elle avait été une entreprise indépendante de Primair, la société S aurait probablement exercé les mêmes fonctions de commercialisation et engagé le même montant de dépenses en la matière.

30. Étant donné que la société $\mathrm{S}$ exerce les fonctions relatives à la commercialisation et supporte les coûts et risques correspondants en vertu d'un accord de long terme qui lui confère des droits exclusifs de distribution pour les montres $\mathrm{R}$, la société $\mathrm{S}$ est susceptible d'enregistrer un bénéfice (ou une perte) découlant de ses activités de commercialisation et de distribution. Aux fins de cet exemple et après examen de données comparables jugées raisonnablement fiables, il est supposé que les bénéfices obtenus par la société $\mathrm{S}$ sont similaires à ceux qu'obtiennent des sociétés de commercialisation ou de distribution indépendantes qui supportent des risques et de coûts comparables durant les premières années de contrats de long terme comparables en matière de commercialisation et de distribution portant sur des produits également inconnus sur le marché.

31. Compte tenu des hypothèses qui précèdent, les revenus de la société $\mathrm{S}$ dans des conditions de pleine concurrence et les activités de commercialisation qu'elle conduit ainsi que les dépenses correspondantes ne diffèrent pas sensiblement des revenus, activités et dépenses propres à des sociétés de commercialisation ou de distribution indépendantes lors de transactions comparables sur le marché libre. Les informations relatives à des accords comparables entre parties indépendantes fournissent le meilleur 
indicateur du revenu de pleine concurrence obtenu par la société S pour évaluer la contribution à la valeur des actifs incorporels découlant des fonctions exercées et des risques et coûts assumés. Ce revenu correspond donc à la rémunération de pleine concurrence pour la société $\mathrm{S}$ au titre de ses contributions et mesure précisément la part revenant à la société $\mathrm{S}$ sur les revenus tirés de l'exploitation de la marque de fabrique et du nom commercial dans le pays Y. Il n'est pas nécessaire de verser à la société $\mathrm{S}$, séparément ou en supplément, une quelconque autre rémunération.

\section{Exemple 11}

32. Aux fins de cet exemple, les faits sont identiques à ceux décrits dans l'exemple 10 , si ce n'est que la société $\mathrm{S}$ mène cette fois des activités de développement commercial plus importantes.

33. Lorsque la société qui assure la commercialisation/distribution supporte effectivement les coûts et les risques associés à ses activités de commercialisation, la question de la répartition des bénéfices potentiels générés par ces activités est soulevée. Une analyse fonctionnelle approfondie permet d'identifier plusieurs entreprises indépendantes qui exercent des fonctions de commercialisation et de distribution similaires en vertu de contrats de long terme comparables en matière de commercialisation et de distribution. Il est néanmoins supposé que les dépenses de commercialisation supportées par la société $\mathrm{S}$ au cours des années 1 à 5 excèdent largement celles engagées par les sociétés de commercialisation et de distribution indépendantes identifiées. Supposons en outre que la société $S$ supporte ces dépenses élevées parce qu'elle exerce des fonctions supplémentaires ou plus intensives que celles des comparables potentiels et que Primair et la société $\mathrm{S}$ escomptent que ces fonctions supplémentaires génèrent des marges ou un chiffre d'affaires plus élevés. $\mathrm{Au}$ vu des activités de développement commercial qu'elle a menées, il apparaît clairement que la société $\mathrm{S}$ a apporté une contribution fonctionnelle plus importante au développement du marché et des actifs incorporels liés à la commercialisation, et qu'elle a assumé des risques et des coûts bien plus importants (en particulier par comparaison avec l'exemple 10) que les entreprises indépendantes potentiellement comparables qui ont été identifiées. Par ailleurs, des éléments conduisent à estimer que les bénéfices obtenus par la société $\mathrm{S}$ sont sensiblement plus faibles que les marges bénéficiaires engrangées par les sociétés de commercialisation et de distribution indépendantes potentiellement comparables lors des années correspondantes de contrats de long terme similaires en matière de commercialisation et de distribution. 
34. Comme dans l'exemple 10, la société $\mathrm{S}$ assume les coûts de ses activités de commercialisation, et les risques correspondants, en vertu d'un contrat de long terme qui lui confère des droits exclusifs de commercialisation et de distribution des montres $\mathrm{R}$ et est susceptible d'enregistrer un bénéfice (ou une perte) découlant de ses activités de commercialisation et de distribution. Cependant, dans ce cas, la société $\mathrm{S}$ a exercé des fonctions de commercialisation et supporté des dépenses allant au-delà des fonctions exercées et des dépenses supportées pour leur propre compte par des entreprises indépendantes disposant de droits similaires lors de transactions potentiellement comparables, ce qui explique que les marges bénéficiaires de la société $\mathrm{S}$ soient sensiblement inférieures à celles obtenues par ces entreprises indépendantes.

35. En l'espèce, il est évident que la société $\mathrm{S}$, qui a exercé des fonctions de commercialisation et supporté des dépenses allant clairement au-delà des fonctions exercées et des dépenses supportées par des sociétés de commercialisation et de distribution indépendantes lors de transactions comparables, n'a pas reçu la rémunération appropriée au travers des marges générées par la revente des montres R. Dans ces circonstances, il serait opportun que l'administration fiscale du pays $\mathrm{Y}$ propose un ajustement des prix de transfert visant à faire en sorte que la rémunération de la société $\mathrm{S}$ au titre des fonctions de commercialisation exercées (compte tenu des risques assumés et des dépenses supportées) soit conforme à ce que des entreprises indépendantes auraient obtenu lors de transactions comparables. Selon les faits et circonstances établis à l'issue d'une analyse de comparabilité détaillée, plusieurs approches sont envisageables pour réaliser un tel ajustement :

- baisser le prix auquel la société $\mathrm{S}$ achète les montres $\mathrm{R}$ auprès de Primair. Un tel ajustement pourrait être pratiqué en appliquant la méthode du prix de revente ou la méthode transactionnelle de la marge nette à partir des données disponibles concernant les bénéfices réalisés par des entreprises de commercialisation et de distribution comparables qui supportent des dépenses de commercialisation et de distribution comparables, si de telles entités comparables peuvent être identifiées ;

- appliquer la méthode du partage des bénéfices résiduels comme approche alternative pour répartir les bénéfices combinés tirés des ventes de montres $\mathrm{R}$ dans le pays $\mathrm{Y}$ en octroyant d'abord à la société $\mathrm{S}$ et à Primair un revenu de base au titre des fonctions exercées, puis en allouant le bénéfice résiduel selon les contributions respectives de la société $\mathrm{S}$ et de Primair à la 
génération des revenus et à la valeur de la marque de fabrique $\mathrm{R}$ et du nom commercial $\mathrm{R}$;

- verser directement à la société $\mathrm{S}$ une rémunération au titre des dépenses de commercialisation qu'elle a supportées au-delà du niveau de dépenses engagées par des entreprises indépendantes comparables, en incluant un élément de bénéfice approprié au titre des fonctions exercées et des risques assumés ayant conduit à supporter ces dépenses.

36. Dans cet exemple, l'ajustement proposé est justifié par le fait que la société $\mathrm{S}$ a exercé des fonctions, assumé des risques et supporté des coûts qui ont contribué au développement des actifs incorporels, en contrepartie de quoi elle n'a pas reçu de rémunération appropriée en vertu de l'accord conclu avec Primair. Si l'accord passé entre Primair et la société S avait prévu que cette dernière pourrait percevoir, pendant la durée restante de l'accord, une rémunération conforme au principe de pleine concurrence au titre des investissements supplémentaires réalisés, une conclusion différente pourrait être justifiée.

\section{Exemple 12}

37. Aux fins de cet exemple, les faits sont identiques à ceux décrits dans l'exemple 10, si ce n'est que la société $\mathrm{S}$ conclut cette fois un contrat de trois ans relatif à la commercialisation et à la distribution des montres $\mathrm{R}$ dans le pays $\mathrm{Y}$, non renouvelable mais ne donnant pas lieu au paiement de redevances. À l'issue de la période de trois ans, la société S ne conclut pas de nouvel accord avec Primair.

38. Supposons qu'il soit démontré que des entreprises indépendantes concluent des contrats de distribution de court terme qui supposent des dépenses de commercialisation et de distribution uniquement si elles ont la perspective de recevoir une rémunération établie en fonction des fonctions exercées, des actifs utilisés et des risques assumés pendant la durée d'effet du contrat. L'observation d'entreprises indépendantes comparables montre qu'elles ne réalisent pas de gros investissements pour mettre en place des infrastructures de commercialisation et de distribution lorsqu'elles concluent seulement un de court terme en matière de commercialisation et de distribution, qui présente par essence un risque de non-renouvellement sans rémunération. Le contrat de commercialisation et de distribution étant potentiellement un accord à court terme, il existe un risque que la société $\mathrm{S}$ ne soit pas en mesure de retirer de bénéfices au titre des dépenses de commercialisation et de distribution qu'elle a engagées à ses propres 
risques. Pour ces mêmes raisons, le bénéfice des efforts réalisés par la société $\mathrm{S}$ pourrait bien être récolté par Primair dans le futur.

39. Les risques assumés par la société $\mathrm{S}$ sont cette fois significativement plus élevés que dans l'exemple 10 et la société $S$ n'a pas reçu de rémunération conforme au principe de pleine concurrence au titre de ces risques supplémentaires. Dans le cas présent, la société $\mathrm{S}$ a conduit des activités de développement commercial et supporté des dépenses de commercialisation allant au-delà des fonctions exercées et des dépenses supportées pour leur compte par des entreprises indépendantes comparables disposant de droits similaires, ce qui explique que les marges bénéficiaires de la société $\mathrm{S}$ soient nettement inférieures à celles obtenues par des entreprises comparables. Le contrat étant un accord à court terme, il ne serait pas raisonnable d'escompter que la société $\mathrm{S}$ puisse percevoir des bénéfices appropriés en vertu du contrat à durée limitée conclu avec Primair. Dans ces circonstances, la société $\mathrm{S}$ devrait recevoir une rémunération au titre de la contribution qu'elle apporte, à ses propres risques, à la valeur de la marque de fabrique $\mathrm{R}$ et du nom commercial $\mathrm{R}$ pendant la durée du contrat conclu avec Primair.

40. Cette rémunération pourrait être versée directement par Primair à la société $\mathrm{S}$ à hauteur de la valeur prévisionnelle générée grâce aux activités de développement commercial de la société $\mathrm{S}$ et aux dépenses de commercialisation correspondantes. Selon une autre approche, l'ajustement pourrait prendre la forme d'une réduction du prix payé par la société $\mathrm{S}$ à Primair pour acheter les montres pendant les années 1 à 3 .

\section{Exemple 13}

41. Aux fins de cet exemple, les faits sont identiques à ceux décrits dans l'exemple 10, avec les circonstances supplémentaires suivantes :

- à la fin de l'année 3, la marque $\mathrm{R}$ est établie avec succès sur le marché du pays $\mathrm{Y}$ et Primair et la société $\mathrm{S}$ renégocient leur accord précédent et concluent un nouveau contrat de licence sur le long terme. Le nouveau contrat prend effet au début de l'année 4 pour une durée de cinq ans et offre à la société $S$ une option pour cinq années supplémentaires. En vertu de ce contrat, la société $S$ s'engage à verser à Primair une redevance calculée en fonction des ventes brutes de montres portant la marque de fabrique R. Toutes les autres dispositions du nouveau contrat sont identiques à celles de l'accord précédent. Le prix payé par la société $\mathrm{S}$ pour l'achat des montres $\mathrm{R}$ n'est pas révisé du fait de l'introduction de la redevance. 
- au cours des années 4 et 5 , les ventes de montres $\mathrm{R}$ par la société $\mathrm{S}$ sont conformes aux prévisions. Cependant, l'introduction de la redevance au début de l'année 4 se traduit par une baisse significative des marges bénéficiaires de la société $\mathrm{S}$.

42. Supposons qu'il n'existe aucune donnée établissant que des sociétés de commercialisation et de distribution indépendantes de produits de marques similaires ont conclu des accords comparables prévoyant le paiement de redevances. Les activités de commercialisation de la société $\mathrm{S}$ et les dépenses correspondantes correspondent, à partir de l'année 4 et par la suite, aux activités et aux dépenses d'entreprises indépendantes.

43. Dans le cadre d'une analyse de prix de transfert, on n'attendrait généralement pas d'une entité de commercialisation et de distribution qu'elle verse une redevance lors de transactions de pleine concurrence alors qu'elle n'obtient aucun droit aux fins de la fixation de prix de transfert sur les marques de fabrique et autres actifs incorporels similaires hormis un droit d'utiliser ces actifs incorporels pour distribuer un produit de marque fourni par l'entité qui perçoit les revenus générés par l'exploitation de ces actifs incorporels. En outre, du fait du paiement de la redevance, les marges bénéficiaires de la société $\mathrm{S}$ sont inférieures à celles obtenues par des entreprises indépendantes, comparables en termes de fonctions exercées, d'actifs utilisés et de risques assumés, au cours de périodes équivalentes de contrats de long terme similaires en matière de commercialisation et de distribution. Les circonstances du cas d'espèce justifieraient donc l'application d'un ajustement de prix de transfert qui interdise le paiement des redevances.

\section{Exemple 14}

44. Aux fins de cet exemple, les faits sont identiques à ceux décrits dans l'exemple 11, avec les circonstances supplémentaires suivantes :

- à la fin de l'année 3, Primair cesse de fabriquer des montres et sous-traite leur production auprès d'un tiers. Dès lors, la société $\mathrm{S}$ importera des montres sans marque auprès du fabriquant et réalisera des opérations de transformation consistant à appliquer le nom et le logo $\mathrm{R}$ et à conditionner les montres avant leur vente au client final. Les montres seront ensuite distribuées et vendues comme décrit dans l'exemple 11 ;

- En conséquence, au début de l'année 4, Primair et la société S renégocient leur accord précédent et concluent un nouveau contrat de licence à long terme. Ce nouveau contrat prend effet au début de 
l'année 4 pour une durée de cinq ans, et offre à la société $\mathrm{S}$ une option pour cinq années supplémentaires.

- En vertu du nouveau contrat, la société S reçoit un droit exclusif, sur le territoire du pays $\mathrm{Y}$, de réaliser les opérations de transformation, de commercialiser et de distribuer les montres $\mathrm{R}$ en contrepartie du versement à Primair d'une redevance calculée en fonction des ventes brutes de montres. La société $\mathrm{S}$ ne reçoit de Primair aucune rémunération au titre de la renégociation de l'accord initial de commercialisation et de distribution. Aux fins de cet exemple, il est supposé que le prix acquitté par la société $\mathrm{S}$ lorsqu'elle achète les montres à compter du début de l'année 4 est un prix de pleine concurrence et que ce prix n'inclut aucune rémunération au titre du nom commercial $\mathrm{R}$.

45. Dans le cadre d'un contrôle fiscal conduit par l'administration fiscale du pays Y au cours de l'année 6, il apparaît, à l'issue d'une analyse fonctionnelle appropriée, que les dépenses de commercialisation supportées par la société $\mathrm{S}$ durant les années 1 à 3 sont largement supérieures à celles supportées par des sociétés de commercialisation et de distribution indépendantes ayant conclu des contrats à long terme similaires en matière de commercialisation et de distribution. Il est également établi que la société $\mathrm{S}$ a mené des activités de commercialisation plus importantes et plus intensives que celles de sociétés de commercialisation et de distribution indépendantes, et que ce surplus relatif d'activité a porté ses fruits et a permis d'accroître les ventes et/ou d'augmenter les marges bénéficiaires globales perçues par le groupe Primair au titre des ventes dans le pays Y. Compte tenu de la portée des activités de commercialisation de la société $\mathrm{S}$ et de son contrôle stratégique sur ces activités, l'analyse de comparabilité, notamment fonctionnelle, conclut clairement que la société $\mathrm{S}$ a supporté des coûts et des risques significativement plus élevés que ceux supportés par des entreprises indépendantes. Il est de plus établi que les marges bénéficiaires individuelles de la société $\mathrm{S}$ sont largement inférieures à celles obtenues par des entreprises indépendantes comparables au cours de périodes équivalentes de contrats de long terme similaires en matière de commercialisation et de distribution.

46. Le contrôle conduit dans le pays Y établit également qu'au cours des années 4 et 5, la société $\mathrm{S}$ supporte les dépenses de commercialisation et les risques correspondants en vertu du nouveau contrat de licence à long terme conclu avec Primair, et que, du fait de cette durée sur le long terme, la société $\mathrm{S}$ la société $\mathrm{S}$ est susceptible d'enregistrer un bénéfice (ou une perte) découlant de ses activités. Cependant, la société $\mathrm{S}$ a conduit des activités de développement commercial et supporté des dépenses de commercialisation 
allant bien au-delà des activités exercées et des dépenses supportées pour leur compte par des entreprises indépendantes comparables ayant conclu des contrats de licence à long terme similaires, ce qui explique que les marges bénéficiaires prévisionnelles de la société $\mathrm{S}$ soient significativement inférieures à celles d'entreprises comparables.

47. Dans ces circonstances, la société $S$ devrait recevoir une rémunération sous la forme d'un revenu supplémentaire au titre des fonctions de développement commercial exercées, des actifs utilisés et des risques assumés. Pour les années 1 à 3 , l'ajustement nécessaire pourrait s'appuyer sur l'approche décrite à l'exemple 11. Pour les années 4 à 5 , l'ajustement pourrait suivre une approche similaire, mais en réduisant les paiements de redevances que la société $\mathrm{S}$ verse à Primair plutôt que de baisser le prix d'achat des montres. Selon les faits et circonstances du cas d'espèce, il conviendrait également d'analyser l'opportunité de verser une rémunération à la société $\mathrm{S}$ au titre de la renégociation de l'accord à la fin de l'année 3, conformément aux recommandations énoncées dans la partie II du chapitre IX.

\section{Exemple 15}

48. Shuyona, société-mère d'un groupe d'entreprises multinationales, est constituée dans le pays $\mathrm{X}$ où elle exerce ses activités. Le groupe Shuyona assure la fabrication et la vente de biens de consommation. Afin de préserver et, si possible, d'accroître ses parts de marché, le groupe Shuyona conduit des activités continues de recherche visant à améliorer les produits existants et à mettre au point de nouveaux produits. Le groupe Shuyona dispose de deux centres de recherche-développement, le premier étant géré par Shuyona dans le pays X, le second par la société S, filiale de Shuyona, qui exerce ses activités dans le pays Y. Le centre de recherchedéveloppement de Shuyona assume la responsabilité du programme de recherche global du groupe Shuyona. Ce centre de recherche-développement conçoit les programmes de recherche, prépare et contrôle les budgets, décide de la localisation des activités de recherche, assure le suivi de l'avancement de tous les projets de recherche-développement et, de manière générale, dirige la fonction de recherche-développement pour le groupe d'entreprises multinationales, en appliquant les orientations stratégiques formulées par la direction du groupe Shuyona.

49. Le centre de recherche-développement de la société $\mathrm{S}$ mène à bien les projets que le centre de recherche-développement de Shuyona lui attribue selon une approche projet par projet. Les propositions émanant du personnel de recherche de la société $\mathrm{S}$ concernant des changements à apporter au programme de recherche doivent être formellement validées par le centre de 
recherche-développement de Shuyona. Le centre de recherchedéveloppement de la société $\mathrm{S}$ transmet des rapports d'avancement de ses activités au moins chaque mois aux responsables du centre de recherchedéveloppement de Shuyona. Si la société $\mathrm{S}$ dépasse le budget alloué à ses travaux par Shuyona, les responsables du centre de recherchedéveloppement de Shuyona doivent approuver tout supplément de dépenses. Les contrats conclus entre les deux centres de recherche-développement précisent que Shuyona supporte toutes les dépenses liées aux activités de recherche-développement de la société $\mathrm{S}$ et tous les risques correspondants. Tous les brevets, modèles et autres actifs incorporels mis au point par le personnel de recherche de la société S sont déposés par Shuyona, en vertu d'accords entre les deux entreprises. Shuyona verse à la société S une commission au titre des activités de recherche-développement de cette dernière.

50. Une analyse des prix de transfert de ce cas d'espèce reconnaîtrait tout d'abord que Shuyona est le propriétaire légal des actifs incorporels. Shuyona contrôle et gère ses propres travaux de recherche-développement et ceux de la société S. Elle assure les fonctions importantes en la matière comme la préparation des budgets et des programmes de recherche, la définition des projets, le financement et le contrôle des dépenses. À ce titre, Shuyona est en droit de percevoir des revenus générés par l'exploitation des actifs incorporels mis au point grâce aux travaux de recherchedéveloppement de la société $\mathrm{S}$. Cette dernière est en droit de percevoir une rémunération au titre des fonctions exercées, des actifs utilisés et des risques assumés. Lors de la détermination de la rémunération due à la société $\mathrm{S}$, il conviendrait de tenir compte de facteurs tels que les compétences et l'efficacité propres au personnel de recherche de la société S, la nature des travaux de recherche entrepris, et d'autres facteurs apportant une contribution à la valeur. Dans la mesure où des ajustements de prix de transfert sont nécessaires pour refléter le montant qu'un fournisseur de services de recherche-développement comparable recevrait au titre de ses prestations, ces ajustements devraient en règle générale porter sur l'année de fourniture du service, sans porter atteinte au droit de Shuyona à percevoir par la suite des revenus générés par l'exploitation des actifs incorporels obtenus grâce aux activités de recherche-développement de la société $\mathrm{S}$.

\section{[Exemple 16}

51. Shuyona, société-mère d'un groupe d'entreprises multinationales, est constituée dans le pays $\mathrm{X}$ où elle exerce exclusivement ses activités. Le groupe Shuyona assure la fabrication et la vente de biens de consommation. Afin de préserver et, si possible, d'accroître ses parts de marché, le groupe Shuyona conduit des activités continues de recherche visant à améliorer les 
produits existants et à mettre au point de nouveaux produits. Le groupe Shuyona dispose de deux centres de recherche-développement, le premier étant géré par Shuyona dans le pays X, le second par la société $\mathrm{S}$, filiale de Shuyona, qui exerce ses activités dans le pays $\mathrm{Y}$.

52. Le groupe Shuyona commercialise deux lignes de produits. Shuyona conduit toutes les activités de recherche-développement relatives à la ligne de produits $\mathrm{A}$, tandis que le centre de recherche-développement de la société $\mathrm{S}$ se charge de la ligne de produits $\mathrm{B}$. La société $\mathrm{S}$ est également le siège régional du groupe Shuyona pour l'Amérique du Nord et assume la responsabilité des activités d'exploitation liées à la ligne de produits $\mathrm{B}$ dans le monde entier. Cependant, tous les brevets découlant des travaux de recherche de la société S sont déposés par Shuyona. Celle-ci ne verse pas de rémunération, ou seulement une rémunération symbolique, à la société $\mathrm{S}$ au titre des inventions brevetables mises au point par le centre de recherchedéveloppement de la société $\mathrm{S}$.

53. Les centres de recherche-développement de Shuyona et de la société $\mathrm{S}$ fonctionnent de manière autonome, chacun d'eux supportant ses propres coûts d'exploitation. Conformément aux orientations générales formulées par la direction du groupe Shuyona, le centre de recherchedéveloppement de la société $\mathrm{S}$ définit ses propres programmes de recherche, établit ses budgets, décide de suspendre ou de modifier des projets de recherche-développement, et recrute son personnel. Le centre de recherchedéveloppement de la société $\mathrm{S}$ rend compte de ses activités aux responsables de la ligne de produit $\mathrm{B}$ au sein de la société $\mathrm{S}$, et non au centre de recherche-développement de Shuyona. Des réunions conjointes entre les équipes de recherche-développement de Shuyona et de la société $\mathrm{S}$ sont organisées ponctuellement pour débattre des méthodes de recherche et examiner des problèmes communs.

54. Une analyse des prix de transfert de ce cas d'espèce reconnaîtrait tout d'abord que Shuyona est le propriétaire légal des actifs incorporels /le titulaire des brevets mis au point par la société $\mathrm{S}$. À la différence de la situation décrite dans l'exemple 15, Shuyona n'assure pas ici le contrôle des fonctions de recherche de la société S, notamment des fonctions importantes de direction, préparation, gestion budgétaire et financement de la recherche. En conséquence, Shuyona est le propriétaire légal des actifs incorporels mais n'est pas en droit de retenir ou de percevoir un quelconque revenu généré par les actifs incorporels relatifs à la ligne de produits $\mathrm{B}$. Les administrations fiscales pourraient établir un prix de transfert approprié en reconnaissant la qualité de Shuyona comme propriétaire légal des actifs incorporels mais en confirmant que la société $S$, compte tenu de ses contributions en termes de fonctions exercées, actifs utilisés et risques assumés, recevra une rémunération appropriée à condition de ne verser ni 
redevance ni paiement quelconque à Shuyona au titre du droit d'utiliser les actifs incorporels que la société $S$ aurait réussi à mettre au point, de manière à ce que les revenus tirés à l'avenir de l'exploitation de ces actifs incorporels par la société $\mathrm{S}$ reviennent à cette dernière et non à Shuyona.

55. Si Shuyona exploite elle-même les actifs incorporels liés à la ligne de produits $\mathrm{B}$, elle devrait verser une rémunération appropriée à la société $\mathrm{S}$ au titre des fonctions exercées, des actifs utilisés et des risques assumés en lien avec la mise au point des actifs incorporels concernés. Lors de la détermination de la rémunération appropriée de la société $S$, du fait que celle-ci assure toutes les fonctions importantes liés à la mise au point des actifs incorporels, il ne serait probablement pas approprié de choisir la société $\mathrm{S}$ comme étant la partie testée dans un accord de prestation de services de recherche-développement.

\section{Exemple 17}

56. Shuyona, société-mère d'un groupe d'entreprises multinationales, est constituée dans le pays $\mathrm{X}$ où elle exerce exclusivement ses activités. Le groupe Shuyona assure la fabrication et la vente de biens de consommation. Afin de préserver et, si possible, d'accroître ses parts de marché, le groupe Shuyona conduit des activités continues de recherche visant à améliorer les produits existants et à mettre au point de nouveaux produits. Le groupe Shuyona dispose de deux centres de recherche-développement, le premier étant géré par Shuyona dans le pays X, le second par la société S, filiale de Shuyona, qui exerce ses activités dans le pays Y. Les relations existant entre les centres de recherche-développement de Shuyona et de la société S sont celles décrites dans l'exemple 15.

57. $\mathrm{Au}$ cours de l'année 1, Shuyona vend tous les droits sur les brevets et sur les autres actifs incorporels de nature technologique, y compris sur les actifs incorporels en cours de mise au point, à une nouvelle filiale, la société $T$, constituée dans le pays $Z$. La société $T$ met en place un site de fabrication dans le pays $Z$ et commence à fournir des produits aux entités du groupe Shuyona à travers le monde. Aux fins de cet exemple, il est supposé que la rémunération versée par la société $T$ en contrepartie du transfert des brevets et des actifs incorporels correspondants reflète le prix de pleine concurrence des actifs incorporels transférés au moment de la transaction.

58. $\mathrm{Au}$ moment du transfert des brevets et des actifs incorporels correspondants, la société $\mathrm{T}$ conclut deux contrats de recherche distincts, le premier avec Shuyona, le second avec la société S. En vertu de ces contrats, la société $\mathrm{T}$ s'engage à assumer les risques financiers liés à un possible échec de projets de recherche-développement à venir, à supporter les coûts 
de toutes les futures activités de recherche-développement, et à verser à Shuyona et à la société $\mathrm{S}$ une commission au titre de services rendus, calculée en fonction du coût des activités de recherche-développement majoré d'un élément de profit équivalent à la marge bénéficiaire sur les coûts obtenue par certaines entreprises indépendantes identifiées qui fournissent des services de recherche.

59. La société $\mathrm{T}$ ne dispose pas du personnel technique en mesure de conduire et de superviser les activités de recherche. Shuyona continue à mettre au point et à définir le programme de recherche-développement relatif à la mise au point des actifs incorporels, à établir ses propres budgets de recherche-développement, à recruter ses équipes de recherche et à décider de suspendre ou de modifier ses projets de recherchedéveloppement. De plus, Shuyona continue à assurer le suivi et le contrôle des activités de recherche-développement de la société $\mathrm{S}$ comme décrit dans l'exemple 15.

60. Une analyse des prix de transfert de ce cas d'espèce reconnaitrait tout d'abord que la société $\mathrm{T}$ est le propriétaire légal des actifs incorporels à la suite du transfert. Shuyona est en droit de percevoir une rémunération au titre des activités de recherche qu'elle exerce ainsi que des fonctions de gestion et de contrôle de ces mêmes activités. La société S est elle aussi en droit de recevoir une rémunération au titre des fonctions de recherche qu'elle exerce. La société $\mathrm{T}$ en droit de recevoir une rémunération au titre de ses fonctions de fabrication et de l'investissement dans les actifs incorporels acquis. La société $\mathrm{T}$ devrait également recevoir une rémunération au titre du financement des travaux de recherche-développement en cours. Il peut s'avérer extrêmement difficile, voire impossible, d'identifier des transactions comparables à une telle organisation et le recours à des méthodes de partage des bénéfices, à des techniques d'évaluation, ou à d'autres méthodes pourra être nécessaire pour identifier la rémunération appropriée due à Shuyona en contrepartie des fonctions exercées, des actifs utilisés et des risques assumés.

\section{Exemple 18}

61. La société A est une société pharmaceutique pleinement intégrée qui exerce des activités de recherche, mise au point, production et vente de préparations pharmaceutiques. La société A conduit ses activités dans le pays X. Dans le cadre de ses activités de recherche, la société A fait régulièrement appel à des sociétés de recherche sous contrat (SRC) pour conduire diverses activités de recherche-développement, notamment la définition et la réalisation d'essais cliniques portant sur les produits en cours de mise au point par la société $\mathrm{A}$. Cependant, les SRC ne mènent pas les 
activités de recherche fondamentale nécessaires pour identifier de nouveaux composés pharmaceutiques. Lorsque la société A fait appel à une SRC pour conduire des activités de recherche clinique, le personnel de recherche de la société A participe activement à la conception des études confiées à la SRC, fournit à celle-ci des informations et des résultats issus de travaux antérieurs, définit les budgets et les échéances pour les projets menés par la SRC et assure un contrôle qualité continu des activités ainsi sous-traitées. Dans le cadre de tels accords, les SRC sont rémunérées sous la forme d'une commission pour services rendus et ne perçoivent aucune part des bénéfices générés par la vente des produits mis au point grâce à leurs travaux de recherche.

62. La société A transfère les brevets et les actifs incorporels liés au produit $\mathrm{M}$, une préparation pharmaceutique se trouvant à un stade peu avancé de mise au point, susceptible d'être utilisée dans le traitement de la maladie d'Alzheimer, à la société $\mathrm{S}$, une filiale de la société A qui exerce ses activités dans le pays $\mathrm{Y}$ (la transaction porte uniquement sur les actifs incorporels existants et n'inclut pas de rémunération au titre des futurs services de recherche-développement de la société A). Aux fins de cet exemple, il est supposé que la société $\mathrm{S}$ paie un prix de pleine concurrence au titre du transfert des actifs incorporels liés au produit $\mathrm{M}$. La société $\mathrm{S}$ ne dispose pas du personnel en mesure de concevoir, de conduire ou de superviser les activités de recherche liées au produit $\mathrm{M}$. La société $\mathrm{S}$ conclut donc un accord avec la société A afin que celle-ci poursuive le programme de recherche en cours lié au produit $\mathrm{M}$ comme elle le faisait avant le transfert des actifs incorporels à la société $\mathrm{S}$. La société $\mathrm{S}$ s'engage à financer toutes les recherches en cours, à assumer le risque financier lié à un échec potentiel de ces recherches, et à payer les services de la société $\mathrm{A}$ selon l'approche du coût majoré, en appliquant la marge générée par des SRC comparables à celles auxquelles la société $\mathrm{A}$ fait régulièrement appel.

63. Une analyse des prix de transfert de ce cas d'espèce reconnaîtrait tout d'abord que, suite au transfert, la société $\mathrm{S}$ est le propriétaire légal des actifs incorporels liés au produit $\mathrm{M}$ en vertu des contrats conclus et des dépôts de brevets effectués. Cependant la société $\mathrm{A}$ continue à gérer et à contrôler les risques liés aux actifs incorporels détenus par la société $\mathrm{S}$, y compris les fonctions importantes décrites au paragraphe 6.56, et est en droit de percevoir une rémunération au titre de ces contributions. Dans ces circonstances, les transactions entre la société A et des SRC ne sont pas comparables aux accords conclus entre la société $\mathrm{S}$ et la société $\mathrm{A}$ en lien avec le produit $\mathrm{M}$ et ne peuvent être utilisées comme référence pour déterminer la rémunération de pleine concurrence revenant à la société $\mathrm{A}$ au titre des activités de recherche-développement en cours concernant les actifs 
incorporels liés au produit M. Dans le cadre de ses transactions avec des SRC, la société A exerce et contrôle certaines fonctions et assume certains risques qui ne sont pas des fonctions exercées et contrôlées, ni des risques assumés, par la société $\mathrm{S}$ lors de ses transactions avec la société $\mathrm{A}$.

64. Bien que la société $\mathrm{S}$ soit propriétaire des actifs incorporels, elle n'est pas en droit de percevoir tous les bénéfices générés par l'exploitation de ceux-ci. La société $\mathrm{S}$ n'étant pas en mesure de contrôler les risques relatifs aux activités de recherche, il convient de reconnaître que la société $\mathrm{A}$ assume une part importante des risques correspondants, et que cette dernière devrait être rémunérée au titre de ces fonctions, notamment des fonctions importantes décrites au paragraphe 6.56. En l'espèce, la société A devrait être en droit de percevoir des revenus supérieurs à ceux qui seraient versés à une SRC et si, comme cela est probable, aucune transaction comparable ne peut être identifiée, il pourra s'avérer nécessaire d'appliquer des méthodes de partage des bénéfices, des techniques d'évaluation, ou d'autres méthodes qui s'appuient moins directement sur des comparables pour déterminer la rémunération appropriée pour la société $\left.\mathrm{A}^{2}{ }^{2}\right]$

\section{Exemple 19}

65. Primarni est une société constituée dans le pays $\mathrm{A}$, où elle exerce ses activités. La société $\mathrm{S}$, une entreprise associée de Primarni, est une société constituée dans le pays $\mathrm{B}$, où elle exerce ses activités. Primarni met au point une invention brevetée et un savoir-faire de fabrication en lien avec le produit X. Elle obtient des brevets valables dans tous les pays concernés dans cet exemple. Primarni et la société S concluent par écrit un contrat de licence par lequel Primarni cède à la société $\mathrm{S}$ le droit d'utiliser les brevets et le savoir-faire liés au produit $\mathrm{X}$ en vue de fabriquer et de vendre ce produit dans le pays $\mathrm{B}$, tandis que Primarni conserve les droits relatifs aux brevets et au savoir-faire en lien avec le produit X pour l'Asie, l'Afrique ainsi que pour le pays $\mathrm{A}$.

66. Supposons que la société $\mathrm{S}$ utilise les brevets et le savoir-faire pour fabriquer le produit $\mathrm{X}$ dans le pays $\mathrm{B}$. Elle vend le produit $\mathrm{X}$ à la fois à des clients indépendants et associés dans le pays B. En outre, elle vend le produit $\mathrm{X}$ à des sociétés de distribution associées implantées en Afrique et en Asie. Les sociétés de distribution associées revendent des unités de produit $\mathrm{X}$ à des clients sur les marchés d'Afrique et d'Asie. Primarni ne fait pas valoir les droits qu'elle a conservés sur les brevets pour l'Asie et l'Afrique qui lui permettraient d'empêcher la société $\mathrm{S}$ de vendre le produit $\mathrm{X}$ à des sociétés de distribution associées exerçant leur activité en Afrique et en Asie. 
67. En l'espèce, le comportement des parties suggère que la transaction réalisée entre Primarni et la société $\mathrm{S}$ est en réalité une cession des droits sur les brevets et savoir-faire relatifs au produit $\mathrm{X}$ dans le pays $\mathrm{B}$, mais aussi en Asie et en Afrique. Lors de l'analyse des prix de transfert portant sur les transactions entre la société S et Primarni, et sur le fondement du comportement des parties, il conviendrait de considérer que les droits cédés à la société $\mathrm{S}$ par le contrat de licence ne se limitent pas au pays $\mathrm{B}$ mais qu'ils s'étendent à l'Asie et à l'Afrique. Le taux de redevance devrait être recalculé pour prendre en compte les ventes totales prévues par la société S sur l'ensemble de ses marchés, y compris celles réalisées par l'intermédiaire des sociétés de distribution implantées en Afrique et en Asie.

\section{Exemple 20}

68. La société $\mathrm{P}$, résidente du pays $\mathrm{A}$, conduit des activités de commerce de détail et exploite plusieurs grands magasins dans le pays A. $\mathrm{Au}$ fil des ans, la société $\mathrm{P}$ a mis au point un savoir-faire particulier et un concept de commercialisation unique en lien avec l'exploitation de ses grands magasins. Il est supposé que le savoir-faire et le concept de commercialisation unique constituent des actifs incorporels au sens de la section $\mathrm{A}$ du chapitre VI. Après avoir conduit ses activités avec succès dans le pays A pendant plusieurs années, la société $\mathrm{P}$ constitue une nouvelle filiale, la société $\mathrm{S}$, dans le pays B. La société $\mathrm{S}$ ouvre de nouveaux grands magasins, qu'elle exploite, dans le pays $\mathrm{B}$, où elle obtient des marges bénéficiaires significativement plus élevées que celles obtenus par des détaillants par ailleurs comparables dans le pays B.

69. Une analyse fonctionnelle détaillée révèle que la société $\mathrm{S}$ utilise, pour conduire ses activités dans le pays B, le savoir-faire et le concept de commercialisation unique utilisés par la société $\mathrm{P}$ dans le pays A. Dans ces circonstances, le comportement les parties révèle qu'une transaction a été réalisée, portant sur le transfert par la société $\mathrm{P}$ vers la société $\mathrm{S}$ du droit d'utiliser le savoir-faire et le concept de commercialisation unique. Dans des circonstances comparables, des parties indépendantes auraient conclu un contrat de licence cédant à la société $\mathrm{S}$ le droit d'utiliser, dans le pays $\mathrm{B}, \mathrm{le}$ savoir-faire et le concept de commercialisation unique mis au point par la société $\mathrm{P}$. En conséquence, une solution possible pour l'administration fiscale consiste à pratiquer un ajustement des prix de transfert sous la forme d'un paiement de redevance par la société $\mathrm{S}$ en faveur de la société $\mathrm{P}$ au titre de l'utilisation de ces actifs incorporels. 


\section{Exemple 21}

70. Ilcha est une société constituée dans le pays A. Depuis de nombreuses années, le groupe Ilcha fabrique et vend le produit $\mathrm{Q}$ dans les pays $\mathrm{B}$ et $\mathrm{C}$ par l'intermédiaire d'une filiale détenue à 100 pour cent, la société $\mathrm{S} 1$, constituée dans le pays $\mathrm{B}$. Ilcha détient des brevets liés à la conception du produit $\mathrm{Q}$ et a mis au point une marque de fabrique unique ainsi que d'autres actifs incorporels relatifs à la commercialisation. Les brevets et marques de fabrique sont déposés au nom de Ilcha dans les pays B et $\mathrm{C}$.

71. Pour des raisons commerciales valables, Ilcha considère que les activités du groupe dans les pays $\mathrm{B}$ et $\mathrm{C}$ se développeraient si elles étaient menées par l'intermédiaire de filiales distinctes dans les pays B et C. En conséquence, Ilcha constitue dans le pays $\mathrm{C}$ une filiale détenue à 100 pour cent, la société $\mathrm{S} 2$. Concernant les activités conduites dans le pays $\mathrm{C}$ :

- la société S1 transfère à la société S2 les actifs corporels relatifs à la fabrication et à la commercialisation des produits qui étaient jusqu'alors utilisés par la société $\mathrm{S} 1$ dans le pays $\mathrm{C}$;

- Ilcha et la société $\mathrm{S} 1$ conviennent de résilier l'accord qui cédait à la société $\mathrm{S} 1$ les droits suivants en lien avec le produit Q : les droits de fabrication et de commercialisation du produit $\mathrm{Q}$ dans le pays $\mathrm{C}$, le droit d'utiliser les brevets et la marque de fabrique dans la conduite de ses activités de fabrication et de commercialisation dans le pays $\mathrm{C}$; le droit d'utiliser des relations avec la clientèle, les listes de clients, la survaleur et d'autres actifs dans le pays $\mathrm{C}$ (ci-après, les « Droits ») ;

- Ilcha conclut avec la société S2 de nouveaux contrats de licence à long terme pour céder à cette dernière les Droits dans le pays $\mathrm{C}$.

La filiale nouvellement constituée mène par la suite les activités liées au produit $\mathrm{Q}$ dans le pays $\mathrm{C}$, tandis que la société $\mathrm{S} 1$ poursuit ses activités liées au produit $\mathrm{Q}$ dans le pays $\mathrm{B}$.

72. Il est supposé qu'au fil des années, les activités de la société S1 dans le pays $\mathrm{C}$ ont atteint une valeur significative et qu'une entreprise indépendante manifeste son intention de racheter ces activités. Il est également supposé qu'aux fins d'une évaluation comptable, industrielle et commerciale, une part de la valeur de ces activités soit considérée comme une survaleur lors de l'affectation du prix d'acquisition réalisée au titre de la vente des activités de la société $\mathrm{S} 1$ dans le pays $\mathrm{C}$ à une partie indépendante. 
73. Compte tenu des faits et circonstances du cas d'espèce, de la valeur est transférée vers la société S2 par la conjonction de deux facteurs : (i) le transfert d'une partie des actifs corporels d'exploitation de la société S1 vers la société S2 dans le pays C et ; (ii) le transfert par Ilcha vers la société $\mathrm{S} 2$ des Droits auxquels la société $\mathrm{S} 1$ a renoncé. On dénombre trois transactions :

- le transfert d'une partie des actifs corporels d'exploitation de la société $\mathrm{S} 1$ vers la société $\mathrm{S} 2$ dans le pays $\mathrm{C}$;

- la restitution par la société S1 des droits cédés par Ilcha ;

- la cession d'une licence par Ilcha à la société S2.

Aux fins de la fixation des prix de transfert, les prix payés par Ilcha et par la société S2 dans le cadre de ces transactions devrait refléter la valeur des activités et donc inclure les sommes pouvant être qualifiées de survaleur lors d'un enregistrement en comptabilité.

\section{Exemple 22}

74. Första, un fabricant de biens de consommation, est une société constituée dans le pays $\mathrm{A}$ où elle exerce son activité. Avant l'année 1, Första produisait le produit $\mathrm{Y}$ dans le pays $\mathrm{A}$ et le vendait dans de nombreux pays à travers le monde par l'intermédiaire de sociétés de distribution affiliées. Le produit $\mathrm{Y}$ bénéficie d'une bonne reconnaissance et se vend à un prix majoré par rapport aux produits concurrents. En effet, Första est le propriétaire légal de la marque de fabrique qu'il a mise au point et dispose d'une survaleur qui se traduit par un tel prix majoré.

75. Au cours de l'année 2, Första constitue dans le pays B la société $\mathrm{S}$, une filiale détenue à 100 pour cent. La société $\mathrm{S}$ intervient comme distributeur principal et centre de facturation. Första continue de livrer le produit $\mathrm{Y}$ directement à ses sociétés de distribution affiliées, mais la propriété des biens est transférée à la société $\mathrm{S}$, qui refacture les produits aux sociétés de distribution affiliées.

76. Au début de l'année 2 , la société $\mathrm{S}$ décide de rembourser aux sociétés de distribution affiliées une part de leurs dépenses publicitaires. Les prix du produit $\mathrm{Y}$ facturés par la société $\mathrm{S}$ à ces distributeurs affiliés sont révisés à la hausse de manière à ce que les marges opérationnelles de ces derniers demeurent constantes malgré le transfert des dépenses publicitaires vers la société $\mathrm{S}$. Il est supposé que les marges opérationnelles obtenues par les distributeurs affiliés sont conformes au principe de pleine concurrence 
aussi bien avant l'année 2 qu'après celle-ci, compte tenu de la hausse des prix des produits et du remboursement des dépenses publicitaires. La société S n'assure aucune fonction en matière publicitaire et n'assume aucun risque lié à la commercialisation des produits.

77. $\mathrm{Au}$ cours de l'année 3, Första baisse les prix facturés à la société S. Första et la société $\mathrm{S}$ affirment que cette baisse est justifiée par le fait que la société $S$ soit désormais en droit de percevoir des revenus liés aux actifs incorporels. Il est avancé que ce revenu est attribuable à des actifs incorporels liés au produit $\mathrm{Y}$ et créés grâce aux dépenses publicitaires supportées par la société $\mathrm{S}$.

78. En substance, la société S n'est pas en droit de percevoir des revenus générés par l'exploitation d'actifs incorporels liés au produit Y. Elle n'exerce aucune fonction, n'assume ni ne contrôle aucun risque, et, en réalité, ne supporte aucune charge liée à la mise au point, à l'amélioration, à la conservation ou à la protection des actifs incorporels. Des ajustements de prix de transfert permettant à Första d'accroître ses revenus au cours de l'année 3 et au-delà seraient donc justifiés.

\section{Exemple 23}

79. La société A détient deux concessions publiques, lui permettant respectivement d'exercer une activité minière et d'exploiter une ligne de chemin de fer. La valeur de marché de la concession minière est égale à 20 et celle de la concession ferroviaire est égale à 10 .

80. Birincil, entreprise indépendante de la société A, acquiert 100 pour cent des titres de participation de la société A pour un prix de 100 . Lors de l'affectation du prix d'acquisition enregistrée par Birincil dans sa comptabilité, une valeur de 20 est allouée à la concession minière, une valeur de 10 est allouée à la concession ferroviaire, et une valeur de 70 est allouée à une survaleur découlant des synergies créées entre les deux concessions.

81. Immédiatement après l'acquisition, Birincil fait en sorte que la société $\mathrm{A}$ transfère ses concessions minière et ferroviaire à la société $\mathrm{S}$, filiale de Birincil.

82. Lors de l'analyse des prix de transfert visant à établir le prix de pleine concurrence devant être payé à la société $\mathrm{S}$ au titre de la transaction avec la société A, il importe d'identifier précisément les actifs incorporels transférés. Au même titre que lors de l'acquisition de la société A par Birincil dans des conditions de pleine concurrence, la survaleur associée aux concessions transférées à la société $\mathrm{S}$ devrait être prise en compte, puisque, 
généralement, on suppose qu'une restructuration des activités internes ne cause ni disparition, ni destruction de valeur.

83. Ainsi, le prix de pleine concurrence de la transaction entre les sociétés $\mathrm{A}$ et $\mathrm{S}$ devrait tenir compte de la concession minière, de la concession ferroviaire et la survaleur reconnue à des fins comptables. Le prix de 100 versé par Birincil pour acheter les titres de participation de la société A représente un prix de pleine concurrence de ce parts et fournit une indication utile sur la valeur combinée des actifs incorporels.

\section{Exemple 24}

84. Birincil acquiert 100 pour cent des titres de participation dans une entreprise indépendante, la société $\mathrm{T}$, pour un prix de 100. La société $\mathrm{T}$ conduit des activités de recherche-développement et a partiellement mis au point plusieurs technologies mais son chiffre d'affaires demeure très limité. Le prix d'acquisition est justifié pour l'essentiel par la valeur de technologies prometteuses, mais dont la mise au point n'est pas achevée, et par la capacité potentielle des salariés de la société $\mathrm{T}$ à poursuivre la mise au point de nouvelles technologies à l'avenir. Lors de l'affectation du prix d'acquisition dans sa comptabilité, Birincil a alloué une valeur de 20 aux actifs corporels et aux actifs incorporels identifiés, et de 80 à la survaleur.

85. Immédiatement après avoir racheté la société $\mathrm{T}$, Birincil fait en sorte que la société $\mathrm{T}$ transfère tous les droits qu'elle détient sur des technologies entièrement ou partiellement mises au point, y compris les brevets, secrets industriels ou commerciaux et savoir-faire techniques, à la société S, filiale de Birincil. En parallèle, la société $\mathrm{S}$ conclut un contrat de recherche avec la société $\mathrm{T}$, qui prévoit que le personnel de cette dernière continuera à se consacrer exclusivement à la mise au point des technologies transférées et au développement de nouvelles technologies pour le compte de la société $\mathrm{S}$. Aux termes de ce contrat, la société $\mathrm{T}$ recevra une rémunération pour ses services de recherche selon le principe du coût majoré d'un élément de profit et tous les droits sur des actifs incorporels mis au point ou améliorés dans le cadre du contrat de recherche reviendront à la société S. En contrepartie, la société S financera toutes les activités de recherche à venir et assumera les risques financiers liés à la possibilité que tout ou partie des activités futures de recherche ne débouche pas sur la mise au point de produits commercialement viables. La société $\mathrm{S}$ dispose d'une équipe de recherche importante, qui comprend des personnels de gestion responsables de technologies de même nature que celles acquises auprès de la société T. À l'issue des transactions décrites ci-dessus, les salariés de la société $\mathrm{S}$ occupant des fonctions de recherche et de gestion assument la pleine responsabilité de la gestion et du suivi des travaux menés par l'équipe 
de recherche de la société $\mathrm{T}$. La société $\mathrm{S}$ approuve les nouvelles activités, prépare et établit des budgets et supervise par ailleurs les travaux de recherche menés par la société $\mathrm{T}$. Tout le personnel de recherche de la société $\mathrm{T}$ conserve le statut de salarié de cette entreprise et se consacre exclusivement à fournir les services prévus au contrat de recherche conclu avec la société $\mathrm{S}$.

86. Lors de l'analyse de prix de transfert visant à déterminer le prix de pleine concurrence que la société $\mathrm{S}$ doit verser à la société $\mathrm{T}$ au titre des actifs incorporels transférés, ainsi que le prix des services de recherche-développement fournis par la société $\mathrm{T}$, il importe d'identifier les actifs incorporels particuliers transférés à la société $\mathrm{S}$ et ceux conservés par le société T. Les définitions et évaluations des actifs incorporels utilisées lors de l'affectation du prix d'acquisition ne sont pas déterminantes aux fins de la fixation des prix de transfert. Le prix de 100 versé par Birincil pour acquérir les titres de participation de la société $\mathrm{T}$ représente un prix de pleine concurrence des titres de participation de cette société et fournit une indication utile concernant la valeur des activités de la société T. La valeur totale de cette activité devrait se refléter soit dans la valeur des actifs corporels et incorporels transférés à la société $\mathrm{S}$, soit dans la valeur des actifs corporels et incorporels et du personnel dont dispose la société $\mathrm{T}$. Dans certains cas, une part importante de la valeur décrite lors de l'affectation du prix d'acquisition comme une survaleur de la société $\mathrm{T}$ peut avoir été transférée à la société $\mathrm{S}$ en même temps que les autres actifs incorporels de la société T. Dans d'autres cas, une partie de la valeur décrite lors de l'affectation du prix d'acquisition comme une survaleur peut avoir été conservée par la société T. Conformément au principe de pleine concurrence, la société $\mathrm{T}$ devrait recevoir une rémunération au titre de cette valeur, qu'elle soit comprise dans le prix payé par la société $S$ au titre du transfert de droits sur des actifs incorporels de nature technologique, ou versée pendant les années qui suivent la transaction au titre des services de recherche-développement fournis par le personnel de la société T. En règle générale, on suppose qu'une restructuration des activités internes ne cause ni disparition, ni destruction de valeur. Si le transfert des actifs incorporels avait eu lieu un certain temps après l'acquisition, une nouvelle analyse aurait été nécessaire pour vérifier si, depuis la date de l'acquisition, les actifs incorporels s'étaient appréciés ou dépréciés.

\section{Exemple 25}

87. Zhu est une société de conseil en développement de logiciels. Par le passé, Zhu a mis au point l'un de ses clients, la banque A, un logiciel qui gère les transactions effectuées auprès des distributeurs automatiques de billets. Dans le cadre de ce projet, Zhu a obtenu et conservé des droits sur un 
code logiciel propriétaire protégé par des droits d'auteur qui serait susceptible de convenir à d'autres clients du secteur bancaire dans la même situation, moyennant toutefois certaines modifications et personnalisations.

88. Il est supposé que la société $\mathrm{S}$, une entreprise associée de Zhu, conclut avec une autre banque, la banque $\mathrm{B}$, un accord distinct portant sur la mise au point d'un logiciel gérant les transactions effectuées auprès des distributeurs automatiques de billets. Zhu accepte d'aider son entreprise associée en lui fournissant des salariés ayant travaillé sur le projet destiné à la banque $\mathrm{A}$ afin qu'ils participent au projet de la société $\mathrm{S}$ destiné à la banque $\mathrm{B}$. Ces salariés ont accès aux éléments de conception logicielle et au savoir-faire mis au point lors du projet destiné à la banque $\mathrm{A}, \mathrm{y}$ compris du code logiciel propriétaire. Ce code et les services fournis par les salariés de Zhu sont utilisés par la société $\mathrm{S}$ pour mener à bien le projet destiné à la banque $\mathrm{B}$. Finalement, la société $\mathrm{S}$ livre à la banque $\mathrm{B}$ un logiciel de gestion des transactions effectuées auprès des distributeurs automatique de billets et la licence correspondante d'utilisation du logiciel développé au cours du projet. Des parties du code propriétaire créé par Zhu dans le cadre du projet destiné à la banque $\mathrm{A}$ sont intégrées dans le logiciel livré par la société $\mathrm{S}$ à la banque $\mathrm{B}$. Ces parties de code créées lors du projet destiné à la banque $\mathrm{A}$ et intégrées dans le logiciel livré à la banque $\mathrm{B}$ sont suffisamment conséquentes pour justifier une réclamation pour violation de droits d'auteur en cas de copie non autorisée par un tiers.

89. Lors d'une analyse des prix de transfert de ces transactions, il conviendrait de prendre en compte deux avantages reçus de la part de Zhu par la société $S$ qui requièrent une rémunération. Premièrement, la société $S$ a reçu des services fournis par les salariés de Zhu mis à sa disposition pour travailler sur le projet destiné à la banque $\mathrm{B}$. Deuxièmement, elle a obtenu des droits sur le logiciel propriétaire de Zhu qui a été utilisé comme socle du développement du logiciel livré à la banque $\mathrm{B}$. La rémunération devant être versée à $Z$ hu par la société $S$ devrait inclure la rémunération due au titre des services et celle correspondant aux droits sur le logiciel.

\section{Exemple 26}

90. Prathamika est la société-mère d'un groupe d'entreprises multinationales. Elle a eu à gérer plusieurs litiges importants et son service juridique interne a acquis une solide expérience dans le suivi de tels dossiers pour le compte de la société. Dans le cadre de la gestion de ces litiges, Prathamika a mis au point des logiciels de gestion documentaire uniques dans son secteur.

91. La société S, entreprise associée de Prathamika, se voit impliquée dans un litige complexe similaire à ceux que le service juridique de 
Prathamika a appris à gérer. Prathamika convient de fournir à la société $\mathrm{S}$ deux personnes appartenant à son service juridique pour la gestion de ce litige. Ces salariés de Prathamika sont responsables de la gestion des documents relatifs au litige et utilisent, à ce titre, le logiciel de gestion documentaire de Prathamika. Cependant, ils n'autorisent pas la société S à utiliser ce logiciel en lien avec d'autres litiges ni à le mettre à la disposition des clients de la société $\mathrm{S}$.

92. Dans ce contexte, il ne serait pas approprié de considérer que Prathamika a transféré des droits sur des actifs incorporels à la société $\mathrm{S}$ dans le cadre de cet accord de service. Toutefois, l'expérience des salariés de Prathamika et leur utilisation d'un logiciel leur permettant de fournir les services convenus de manière plus efficace et efficiente sont des éléments qui devraient entrer en ligne de compte aux fins d'une analyse de comparabilité portant sur la fixation d'une commission au titre de services rendus par le personnel mis à disposition par Prathamika.

\section{Exemple 27}

93. Osnovni, société-mère d'un groupe d'entreprises multinationales, a pour activité la mise au point et la vente de logiciels. Elle acquiert 100 pour cent des participations dans la société $\mathrm{S}$, une société cotée constituée dans le même pays qu'Osnovni, pour un prix de 160. Lors de l'acquisition, la valeur de marché totale des actions de la société $\mathrm{S}$ était égale à 100. Les autres acheteurs potentiels de la société $\mathrm{S}$ offraient des montants compris entre 120 et 130 pour le rachat de la société.

94. À la date de son rachat, la société $\mathrm{S}$ n'avait comptabilisé qu'un faible montant d'immobilisations. Sa valeur correspondait pour l'essentiel à des droits sur des actifs incorporels mis au point ou en cours de mise au point liés à des logiciels et aux compétences de son personnel. Lors de l'affectation du prix d'acquisition dans sa comptabilité, Osnovni a alloué une valeur de 10 aux actifs corporels, de 60 aux actifs incorporels, et de 90 à la survaleur. Dans les présentations faites devant son conseil d'administration, Osnovni a justifié le prix d'acquisition de 160 par la complémentarité entre les produits actuels du groupe Osnovni et les produits actuels et potentiels de la société $\mathrm{S}$.

95. La société $\mathrm{T}$ est une filiale détenue à 100 pour cent par Osnovni. Osnovni a toujours octroyé à la société $\mathrm{T}$ des licences exclusives sur tous ses actifs incorporels liés aux marchés européens et asiatiques. Aux fins de cet exemple, il est supposé que tous les accords de licence concernant les marchés européens et asiatiques octroyés à la société $\mathrm{T}$ avant l'acquisition étaient conformes au principe de pleine concurrence. 
96. Immédiatement après avoir racheté la société $\mathrm{S}$, Osnovni procède à sa liquidation et cède à la société $\mathrm{T}$ une licence exclusive et perpétuelle relative aux droits sur les actifs incorporels liés aux produits de la société $\mathrm{S}$ vendus sur les marchés européens et asiatiques.

97. Lors de la détermination du prix de pleine concurrence des actifs incorporels de la société $\mathrm{S}$ cédés sous licence à la société $\mathrm{T}$ en vertu des accords mentionnés ci-dessus, il importe de tenir compte de la surcote des actions de la société $\mathrm{S}$ comprise dans le prix d'acquisition. Dans la mesure où cette surcote traduit la complémentarité entre les produits du groupe Osnovni et ceux de la société S sur les marchés européens et asiatiques dont la société $\mathrm{T}$ a obtenu les licences, le prix acquitté par cette dernière au titre des actifs incorporels et droits sur des actifs incorporels de la société $\mathrm{S}$ qui lui sont transférés devrait refléter une portion appropriée de la surcote d'acquisition. En revanche, dans la mesure où cette surcote est attribuable exclusivement à des complémentarités entre des produits vendus en dehors des marchés de la société $T$, la surcote ne devrait pas entrer en ligne de compte pour déterminer le prix de pleine concurrence payé par la société $\mathrm{T}$ au titre des actifs incorporels de la société $\mathrm{S}$ liés aux marchés géographiques de la société $T$. La valeur attribuée aux actifs incorporels lors de l'affectation du prix d'acquisition à des fins comptables n'est pas déterminante aux fins de la fixation des prix de transfert.

\section{Exemple 28}

98. La société A, société-mère d'un groupe d'entreprises multinationales, exerce ses activités dans le pays X. Elle détient des brevets, des marques de fabrique et des savoir-faire relatifs à plusieurs produits fabriqués et vendus par le groupe. La société B est une filiale détenue à 100 pour cent par la société A. La société B, qui conduit l'ensemble de ses activités dans le pays $\mathrm{Y}$, détient également des brevets, des marques de fabrique et des savoir-faire relatifs au produit $\mathrm{M}$.

99. Pour des raisons commerciales valables liées à la protection des brevets du groupe et aux efforts en matière de lutte contre la contrefaçon, le groupe d'entreprises multinationales décide de centraliser la propriété de ses brevets au sein de la société A. En conséquence, la société B vend les brevets relatifs au produit $\mathrm{M}$ à la société $\mathrm{A}$ pour un montant forfaitaire. Suite à la vente, il incombe à la société $\mathrm{A}$ d'assurer la continuité de toutes les fonctions, et d'assumer et contrôler tous les risques liés aux brevets portant sur le produit M. À l'issue d'une analyse de comparabilité, notamment fonctionnelle, approfondie, le groupe d'entreprises multinationales conclut qu'il n'est pas en mesure d'identifier des transactions comparables sur le marché libre pouvant être utilisées pour fixer le prix de pleine concurrence. 
La société $\mathrm{A}$ et la société $\mathrm{B}$ concluent pour des raisons valables que le recours à des techniques d'évaluation constitue la méthode de prix de transfert la plus appropriée pour déterminer si le prix convenu est conforme à celui de transactions de pleine concurrence.

100. Les évaluateurs utilisent une approche qui évalue directement les actifs et les brevets pour parvenir à une valeur actuelle nette après impôts égale à 80 pour le brevet relatif au produit $\mathrm{M}$. L'analyse est fondée sur des taux de redevance, des taux d'actualisation et des durées d'utilité habituellement pratiqués dans le secteur dont le produit $\mathrm{M}$ est issu. Toutefois, des différences importantes existent entre le produit $\mathrm{M}$ et les droits de brevets relatifs au produit $\mathrm{M}$, d'une part, et les valeurs habituellement pratiquées dans le secteur, de l'autre. Les accords de redevance utilisés lors de l'analyse ne répondraient donc pas aux critères de comparabilité définis aux fins de la méthode du prix comparable sur le marché libre. L'évaluation a pour finalité d'effectuer des ajustements au titre de ces différences.

101. Lors de son évaluation, la société A réalise une analyse fondée sur l'actualisation des flux de trésorerie générés par l'ensemble des activités liées au produit M. Selon cette analyse, qui s'appuie sur les paramètres d'évaluation habituellement employés par la société A pour évaluer des projets d'acquisitions, la valeur actuelle de l'ensemble des activités liées au produit $\mathrm{M}$ est égale à 100 . L'écart de 20 qui sépare l'évaluation de l'ensemble des activités liées au produit $\mathrm{M}$, établie à 100 , de celle du seul brevet, établie à 80 , ne semble pas appropriée pour refléter la valeur actuelle nette des rémunérations correspondant aux fonctions normalement exercées par la société $\mathrm{B}$ et pour rendre compte de la valeur des marques de fabrique et des savoir-faire détenus par la société B. Dans cette situation, il conviendrait de réexaminer la fiabilité de la valeur de 80 attribuée au brevet.

\section{Exemple 29}

102. La société $\mathrm{A}$, société-mère d'un groupe d'entreprises multinationales, exerce ses activités dans le pays $\mathrm{S}$. Les sociétés $\mathrm{B}$ et $\mathrm{C}$ appartiennent à ce même groupe et conduisent leurs activités respectivement dans le pays $\mathrm{T}$ et le pays U. Pour des raisons commerciales valables, le groupe d'entreprises multinationales décide de rassembler en un lieu unique tous ses actifs incorporels liés à des activités conduites hors du pays S. En conséquence, les actifs incorporels détenus par la société $\mathrm{B}$ sont vendus à la société $\mathrm{C}$ pour un montant forfaitaire, incluant les brevets, marques de fabrique, savoir-faire et les relations avec les clients. En parallèle, la société $\mathrm{C}$ choisit la société $\mathrm{B}$ comme sous-traitant chargé de fabriquer les produits que la société $\mathrm{B}$ fabriquait et vendait auparavant en assumant tous 
les risques correspondants. La société $\mathrm{C}$ dispose des ressources et des effectifs requis pour gérer les lignes d'activité acquises, notamment les phases ultérieures de mise au point des actifs incorporels nécessaires à l'activité de la société B.

103. Le groupe d'entreprises multinationales ne parvient pas à identifier des transactions comparables sur le marché libre pouvant être utilisées lors d'une analyse de prix de transfert visant à établir le prix de pleine concurrence versé par la société $\mathrm{C}$ à la société $\mathrm{B}$. Sur le fondement d'une analyse détaillée de comparabilité, notamment fonctionnelle, le groupe d'entreprises multinationales conclut que la méthode de prix de transfert la plus appropriée passe par des techniques d'évaluation des actifs incorporels transférés. Lorsqu'il réalise son évaluation, le groupe n'est pas en mesure de séparer de manière fiable les flux de trésorerie attribuables à chaque actif incorporel concerné.

104. Dans cette situation, il pourrait être pertinent de déterminer la rémunération de pleine concurrence que devra verser la société $\mathrm{C}$ au titre des actifs incorporels vendus par la société B en évaluant les actifs incorporels transférés selon une approche agrégée plutôt qu'en visant une évaluation actif par actif. Cela s'applique tout particulièrement en cas de différence significative entre, d'une part, la somme des meilleures estimations disponibles de la valeur des actifs incorporels et d'autres actifs identifiés et évalués séparément et, d'autre part, la valeur de l'entreprise dans son ensemble.

\section{Exemple 30}

105. Pervichnyi, société-mère d'un groupe d'entreprises multinationales, est constituée dans le pays $\mathrm{X}$ où elle exerce ses activités. Avant l'année 1, Pervichnyi a mis au point les brevets et marques de fabrique concernant le produit $\mathrm{F}$. Elle fabriquait le produit $\mathrm{F}$ dans le pays $\mathrm{X}$ et le fournissait à des filiales de distribution à travers le monde. Aux fins du présent exemple, il est supposé que les prix facturés aux filiales de distribution étaient de façon constante des prix de pleine concurrence.

106. Au début de l'année 1 , Pervichnyi crée une filiale détenue à 100 pour cent, la société $\mathrm{S}$, dans le pays $\mathrm{Y}$. Afin de réduire ses coûts, Pervichnyi transfère la totalité de la fabrication du produit $\mathrm{F}$ à la société $\mathrm{S}$. Lors de la constitution de la société S, Pervichnyi vend à celle-ci les brevets et marques de fabrique concernant le produit $\mathrm{F}$ pour un montant forfaitaire. Dans ces circonstances, Pervichnyi et la société $\mathrm{S}$ cherchent à identifier le prix de pleine concurrence des actifs incorporels transférés en utilisant une méthode d'évaluation fondée sur l'actualisation des flux de trésorerie. 
107. Selon cette évaluation, Pervichnyi aurait pu générer des flux de trésorerie résiduels (après avoir rémunéré dans des conditions de pleine concurrence toutes les fonctions exercées par les autres membres du groupe d'entreprises multinationales) après impôts d'une valeur actuelle de 600 si elle avait continué à fabriquer le produit $\mathrm{F}$ dans le pays $\mathrm{X}$. Du point de vue de l'acheteur, l'évaluation indique que la société $\mathrm{S}$ pourrait générer des flux de trésorerie résiduels après impôts d'une valeur actuelle égale à $1100 \mathrm{si}$ elle détenait les actifs incorporels et fabriquait le produit dans le pays Y. Plusieurs facteurs permettent d'expliquer la différence entre la valeur actuelle des flux de trésorerie résiduels après impôts estimés respectivement pour Pervichnyi et pour la société S.

108. Une autre option offerte à Pervichnyi consisterait à conserver la propriété des actifs incorporels et de confier à la société $\mathrm{S}$ ou à un autre fournisseur la fabrication des produits pour son compte dans le pays Y. Selon ce scénario, Pervichnyi estime qu'elle pourrait générer des flux de trésorerie résiduels après impôts d'une valeur actuelle de 875 .

109. Lors de la détermination de la rémunération de pleine concurrence au titre des actifs incorporels transférés par Pervichnyi à la société $\mathrm{S}$, il importe de tenir compte du point de vue de chacune des parties, des options réalistes qui s'offrent à elles et des faits et circonstances du cas d'espèce. Pervichnyi ne vendrait certainement pas les actifs incorporels à un prix qui génèrerait des flux de trésorerie résiduels après impôts d'une valeur actuelle inférieure à 600 , soit les flux de trésorerie résiduels qu'elle pourrait générer en conservant les actifs incorporels et en poursuivant son activité comme par le passé. De plus, rien ne permet de penser que Pervichnyi vendrait les actifs incorporels pour un prix qui génèrerait des flux de trésorerie résiduels après impôts d'une valeur actuelle inférieure à 875 . Si Pervichnyi pouvait bénéficier de la réduction des coûts de fabrication en chargeant autre entité de produire pour son compte dans un pays à bas coûts, une option réaliste qui s'offre à elle consiste à conduire une telle activité de sous-traitance de fabrication. Cette option réaliste doit entrer en ligne de compte lors de la détermination du prix de vente des actifs incorporels concernés.

110. On ne devrait pas attendre de la société $\mathrm{S}$ qu'elle acquitte un prix qui génèrerait, compte tenu de l'ensemble des faits et circonstances du cas d'espèce, un revenu après impôts inférieur à celui qu'elle obtiendrait en s'abstenant de conclure la transaction. Selon l'évaluation fondée sur l'actualisation des flux de trésorerie, la valeur actuelle nette des flux de trésorerie résiduels après impôts qu'elle pourrait générer en utilisant les actifs incorporels dans le cadre de ses activités serait de 1 100. Les parties pourraient négocier un prix qui permette à Pervichnyi d'obtenir un revenu égal ou supérieur à celui que génèreraient les autres options envisageables, et qui offre à la société $\mathrm{S}$ un retour sur investissement positif compte tenu de 
l'ensemble des faits du cas d'espèce, notamment l'imposition à laquelle la transaction elle-même sera soumise.

111. Une analyse de prix de transfert fondée sur une actualisation des flux de trésorerie doit examiner la manière dont des entreprises indépendantes opérant dans des conditions de pleine concurrence tiendraient compte des réductions de coûts et des effets prévisionnels de la fiscalité pour fixer le prix des actifs incorporels. Ce prix devrait, toutefois, être inclus dans la plage séparant le prix qui permettrait à Pervichnyi d'obtenir des flux de trésorerie résiduels après impôts équivalents à ceux que génèreraient les autres options réalistes dont elle dispose, du prix offrant à la société $\mathrm{S}$ un retour sur investissement positif compte tenu des risques assumés et de l'imposition à laquelle la transaction elle-même serait soumise.

112. Les faits décrits dans cet exemple et l'analyse présentée ont été bien entendu largement simplifiés par rapport à l'analyse nécessaire dans le cas d'une transaction réelle. Cependant, l'analyse illustre bien l'importance de prendre en compte l'ensemble des faits et circonstances du cas d'espèce lors d'une approche fondée sur la valeur actuelle des flux de trésorerie; d'étudier le point de vue de chacune des parties lors d'une telle analyse ; et de tenir compte des options réalistes qui s'offrent aux parties aux fins de l'analyse de prix de transfert.

\section{[Exemple 31}

113. Les droits de fabrication et de distribution d'un médicament mis sur le marché font l'objet d'une licence entre des entreprises associées en vertu d'un accord fixant le taux de la redevance pendant la durée de l'accord, qui est de trois ans. Ces conditions apparaissent conformes aux accords conclus dans des conditions de pleine concurrence au sujet de produits comparables, et le taux est accepté comme étant équivalent à celui qui serait convenu dans le cadre de transactions sur le marché libre fondées sur les bénéfices auxquels les deux parties pourraient raisonnablement s'attendre au moment où l'accord est conclu.

114. $\mathrm{Au}$ cours de la troisième année d'application de l'accord, on découvre que le médicament a des propriétés qui relèvent d'une autre catégorie thérapeutique en combinaison avec un autre médicament et cette découverte aboutit à une augmentation considérable des ventes et des bénéfices du titulaire de la licence. Si l'accord avait été négocié dans des conditions de pleine concurrence au cours de la troisième année en disposant de cette information, il est incontestable qu'un taux de redevance plus élevé aurait été convenu afin de tenir compte de l'accroissement de la valeur de l'actif incorporel. 
115. On dispose d'informations montrant (et ces informations sont transmises à l'administration fiscale) que les nouvelles propriétés du médicament étaient imprévues au moment où l'accord a été conclu et que le taux de redevance fixé au cours de l'année 1 était fondé de manière appropriée sur les bénéfices auxquels les deux parties pouvaient raisonnablement s'attendre à l'époque. L'absence de clause d'ajustement des prix ou d'autres protections contre le risque d'incertitude de l'évaluation est également conforme aux conditions dans lesquelles s'effectuent des transactions comparables sur le marché libre. Par ailleurs, sur la base d'une analyse du comportement d'entreprises indépendantes dans des circonstances similaires, il n'y a pas de raison de croire que l'évolution intervenue au cours de l'année 3 était si fondamentale qu'elle aurait entraîné dans une situation de pleine concurrence une renégociation du prix de la transaction.

116. Compte tenu de toutes ces circonstances, il n'y a pas de raison d'ajuster le taux de la redevance au cours de l'année 3.Un tel ajustement serait contraire aux principes énoncés au chapitre VI dans la mesure où il constituerait dans ce cas un ajustement a posteriori injustifié. Voir paragraphe 6.179. Il n'y a pas de raison de considérer que l'évaluation était suffisamment incertaine au départ et que des parties opérant dans des conditions de pleine concurrence auraient dû exiger une clause d'ajustement de prix ou que le changement de valeur constituait un fait si fondamental qu'il aurait entraîné une renégociation de la transaction. Voir paragraphes 6.180 et 6.181 .

\section{Exemple 32}

117. Les faits sont les mêmes que dans l'exemple précédent. Il est supposé qu'à la fin de la période triennale, l'accord a été renégocié entre les parties. À ce stade, on sait que les droits sur le médicament ont une valeur nettement plus élevée qu'il semblait au départ. Toutefois, l'évolution inattendue de l'année précédente est encore récente et il n'est pas possible de dire avec certitude si les ventes vont continuer à progresser, si d'autres effets bénéfiques vont être découverts, et quels sont les faits intervenus sur le marché qui pourraient affecter les ventes à mesure que les entreprises concurrentes cherchent également à tirer profit de la découverte. Toutes ces considérations font de la réévaluation des droits sur des actifs incorporels un processus très incertain. Néanmoins, les entreprises associées concluent un nouvel accord de licence pour une durée de 10 ans qui a pour effet d'accroître sensiblement le taux fixé pour la redevance sur la base d'anticipations incertaines de maintien et d'accroissement de la demande. 
118. Dans ce secteur, les entreprises n'ont pas l'habitude de conclure des accords à long terme pour fixer les taux de redevance lorsque l'actif incorporel concerné est susceptible d'avoir une valeur élevée, mais cette valeur n'a pas été déterminée en retraçant les transactions effectuées dans le passé. Il n'est pas non plus prouvé qu'étant donné l'incertitude de l'évaluation, des projections effectuées par les entreprises associées auraient été considérées comme suffisantes par des entreprises indépendantes pour justifier la conclusion d'un un accord prévoyant un taux de redevance fixe. On peut admettre que des entreprises indépendantes auraient insisté pour bénéficier d'une protection sous forme de clauses d'ajustement futures des prix sur la base d'examens effectués chaque année.

119. On suppose qu'au cours de l'année 4, les ventes ont augmenté et que le taux de redevance fixé en vertu de l'accord portant sur une période de dix ans est considéré comme conforme au principe de pleine concurrence. Toutefois, au début de l'année 5, un concurrent lance un médicament plus efficace que le premier dans la catégorie thérapeutique dans laquelle le premier médicament combiné à d'autres avait eu des vertus inattendues et les ventes du premier médicament effectuées pour cet usage diminuent rapidement. Le taux de redevance fixé au départ de l'accord décennal ne peut être considéré comme conforme au principe de pleine concurrence audelà de l'année 5 et l'administration fiscale peut à bon droit opérer un ajustement du prix de transfert à partir du début de l'année 6. Cet ajustement est justifié par le fait qu'il apparaît clairement, comme on l'a indiqué au paragraphe précédent, que dans des circonstances comparables des entreprises indépendantes auraient prévu dans l'accord un ajustement de prix fondé sur un examen annuel. Voir paragraphe 6.183.

\section{Exemple 33}

120. Il est supposé que la société $\mathrm{X}$ cède une licence du droit de produire et de distribuer une microplaquette à la société $\mathrm{Y}$, filiale nouvelle créée, pendant une période de cinq ans. Le taux de redevance est fixé à 2 pour cent. Ce taux de redevance est fondé sur une projection des bénéfices susceptibles d'être tirés de l'exploitation de l'actif incorporel, qui fait apparaître des prévisions de ventes de 50 à 100 millions pour chacune des cinq premières années.

121. Il est établi que des contrats entre des entreprises indépendantes portant sur des actifs incorporels comparables dans des circonstances comparables ne considéreraient pas les prévisions comme suffisamment fiables pour justifier un taux de redevance fixe, et que par conséquent les parties conviendraient d'une clause d'ajustement des prix pour tenir compte des différences entre les bénéfices effectifs et les bénéfices prévus. Un 
accord conclu par la société $\mathrm{X}$ avec un fabricant indépendant pour un actif incorporel comparable dans des circonstances comparables et des conditions d'incertitude comparables prévoit les ajustements de taux suivants :

\begin{tabular}{|l|c|}
\hline \multicolumn{1}{|c|}{ Ventes } & Taux \\
\hline Jusqu'à 100 millions & $2,00 \%$ \\
\hline 100 à 150 millions & $2,25 \%$ \\
\hline 150 à 200 millions & $2,50 \%$ \\
\hline Plus de 200 millions & $2,75 \%$ \\
\hline
\end{tabular}

122. En fait, bien que les ventes effectuées par Y au cours de l'année 1 soient de 50 millions, au cours des années ultérieures les ventes sont trois fois supérieures aux chiffres prévus. Conformément aux principes de cette section, pour ces années ultérieures, l'administration fiscale aurait pu à bon droit déterminer le taux de redevance sur la base de la clause d'ajustement qui serait prévue dans le cadre de transactions comparables sur le marché libre telle que celle qui a été conclue entre la société $\mathrm{X}$ et le fabricant indépendant. Voir paragraphes $6.180,6.182$, et 6.183 .] 


\section{Notes}

1. Aux fins de cet exemple, il n'est pas nécessaire de calculer ces résultats. Il est supposé dans le cadre de cet exemple qu'un « investissement» consistant à financer à hauteur de 100 millions USD par an pendant 5 ans un projet présentant un tel niveau de risque devrait déboucher, dans des conditions de pleine concurrence, sur des bénéfices anticipés de 110 millions USD par an pendant les 10 années suivantes.

2. Les délégués de certains pays considèrent que les situations décrites dans les exemples 17 et 18 pourraient être correctement traitées dans certains cas en ignorant les transactions ou en les requalifiant conformément aux dispositions du paragraphe 1.65 puisque ces transactions n'auraient normalement pas lieu entre entreprises indépendantes. Le Plan d'action concernant le BEPS appelle un réexamen des cas dans lesquels il est approprié d'ignorer ou de requalifier des transactions. Ces exemples seront donc soumis à une nouvelle analyse dans le cadre des travaux menés en 2015 au titre du Plan d'action concernant le BEPS. 



\section{ORGANISATION DE COOPÉRATION ET DE DÉVELOPPEMENT ÉCONOMIQUES}

L'OCDE est un forum unique en son genre où les gouvernements œuvrent ensemble pour relever les défis économiques, sociaux et environnementaux liés à la mondialisation. À l'avantgarde des efforts engagés pour comprendre les évolutions du monde actuel et les préoccupations qu'elles suscitent, l'OCDE aide les gouvernements à y faire face en menant une réflexion sur des thèmes tels que le gouvernement d'entreprise, l'économie de l'information et la problématique du vieillissement démographique. L'Organisation offre aux gouvernements un cadre leur permettant de confronter leurs expériences en matière d'action publique, de chercher des réponses à des problèmes communs, de recenser les bonnes pratiques et de travailler à la coordination des politiques nationales et internationales.

Les pays membres de l'OCDE sont: l'Allemagne, l'Australie, l'Autriche, la Belgique, le Canada, le Chili, la Corée, le Danemark, l'Espagne, l'Estonie, les États-Unis, la Finlande, la France, la Grèce, la Hongrie, l'Irlande, l'Islande, Israël, l'Italie, le Japon, le Luxembourg, le Mexique, la Norvège, la Nouvelle-Zélande, les Pays-Bas, la Pologne, le Portugal, la République slovaque, la République tchèque, le Royaume-Uni, la Slovénie, la Suède, la Suisse et la Turquie. L'Union européenne participe aux travaux de l'OCDE.

Les Éditions OCDE assurent une large diffusion aux travaux de l'Organisation. Ces derniers comprennent les résultats de l'activité de collecte de statistiques, les travaux de recherche menés sur des questions économiques, sociales et environnementales, ainsi que les conventions, les principes directeurs et les modèles développés par les pays membres.

ÉDITIONS OCDE, 2, rue André-Pascal, 75775 PARIS CEDEX 16

(23 2014292 P) ISBN 978-92-64-22533-6 - 2014-01 


\section{Projet OCDE/G20 sur l'érosion de la base d'imposition et le transfert de bénéfices}

\section{Instructions relatives aux aspects intéressant les prix de transfert des actifs incorporels}

Endiguer l'érosion de la base d'imposition et le transfert de bénéfices (BEPS) est une priorité absolue pour les pouvoirs publics des pays du monde entier. En 2013, les pays de l'OCDE et du G20 ont adopté un Plan d'action en 15 points, à l'élaboration duquel ils ont œuvré de concert et sur un pied d'égalité, pour lutter contre l'érosion de la base d'imposition et le transfert de bénéfices. Ce rapport présente les résultats obtenus au titre de l'Action 8.

Le Projet BEPS, réalisé sous l'égide de l'OCDE et du G20, vise à assurer aux États des recettes budgétaires grâce à une fiscalité en phase avec l'évolution des activités économiques et la création de valeur, mais aussi à créer, dans le domaine de la fiscalité internationale, un ensemble unique de règles faisant l'objet d'un consensus pour combattre l'érosion de la base d'imposition et le transfert de bénéfices et, partant, à protéger l'assiette imposable tout en offrant aux contribuables une prévisibilité et une certitude accrues. L'un des axes majeurs de l'effort engagé est l'élimination de la double non-imposition. Les nouvelles règles qui doivent être adoptées à cet effet ne doivent toutefois pas entraîner l'application d'une double imposition, soumettre les contribuables à des obligations trop contraignantes ou instituer des restrictions faisant obstacle à des activités transnationales par ailleurs légitimes.

\section{Sommaire}

Synthèse

Modifications apportées aux chapitres I et II des Principes applicables en matière de prix de transfert

Modifications apportées au chapitre VI des Principes applicables en matière de prix de transfert

Chapitre VI. Considérations particulières applicables aux actifs incorporels Annexe apportée au chapitre VI. Exemples d'application des instructions concernant les considérations particulières applicables aux actifs incorporels

\section{http://www.oecd.org/fr/fiscalite/beps.htm}

\section{Veuillez consulter cet ouvrage en ligne : http://dx.doi.org/10.1787/9789264225350-fr.}

Cet ouvrage est publié sur OECD iLibrary, la bibliothèque en ligne de l'OCDE, qui regroupe tous les livres, périodiques et bases de données statistiques de l'Organisation. Rendez-vous sur le site www.oecd-ilibrary.org pour plus d'informations. 